\title{
Maximal regularity for a free boundary problem
}

\author{
Joachim ESCHER \\ Institute of Mathematics, University of Basel \\ 4051 Basel, Switzerland \\ Gieri SIMONETT* \\ Department of Mathematics, Vanderbilt University \\ Nashville, TN 37240, U.S.A.
}

\begin{abstract}
This paper is concerned with the motion of an incompressible fluid in a rigid porous medium of infinite extent. The fluid is bounded below by a fixed, impermeable layer and above by a free surface moving under the influence of gravity. The laminar flow is governed by Darcy's law.

We prove existence of a unique maximal classical solution, using methods from the theory of maximal regularity, analytic semigroups, and Fourier multipliers. Moreover, we describe a state space which can be considered as domain of parabolicity for the problem under consideration.
\end{abstract}

\section{Introduction and main result}

In this paper we investigate a class of free boundary problems, which can be described as follows. Let

$$
\widetilde{\mathfrak{A}}:=\left\{f \in B C^{2}(\mathbb{R}) ; \inf _{x \in \mathbb{R}} f(x)>0\right\} .
$$

Given $f \in \widetilde{\mathfrak{A}}$, define

$$
\Omega_{f}:=\{(x, y) \in \mathbb{R} \times(0, \infty) ; 0<y<f(x)\}
$$

\footnotetext{
* Supported by Schweizerischer Nationalfonds
} 


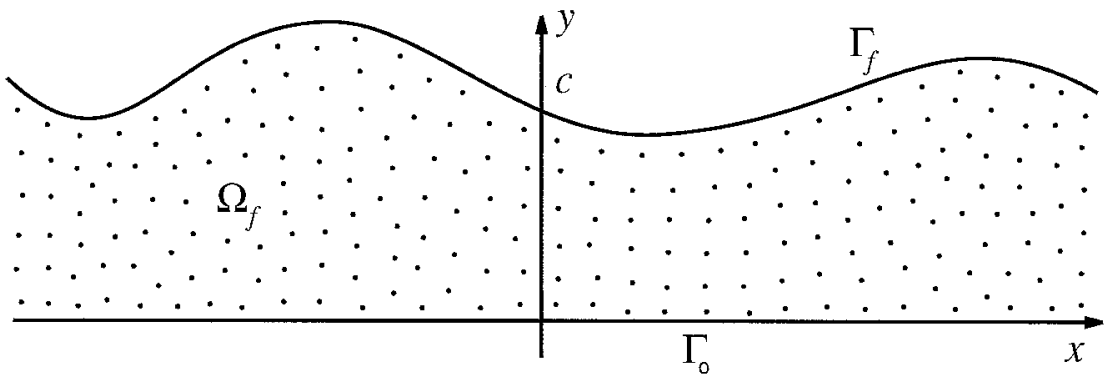

The boundaries of $\Omega_{f}$ are given by

$$
\begin{aligned}
& \Gamma_{0}:=\mathbb{R} \times\{0\}, \\
& \Gamma_{f}:=\operatorname{graph}(f):=\{(x, y) \in \mathbb{R} \times(0, \infty) ; y=f(x)\} .
\end{aligned}
$$

For fixed $T>0$, let $J:=[0, T)$ and $\dot{J}:=J \backslash\{0\}$. Then we address the following problem: Given $f_{0} \in \widetilde{\mathfrak{A}}$ and $c>0$, find a pair of functions $(u, f)$ possessing the regularity

$$
\begin{aligned}
& f \in C(J, \widetilde{\mathfrak{A}}) \cap C^{1}\left(J, B C^{1}(\mathbb{R})\right), \\
& u(t, \cdot) \in B C^{2}\left(\bar{\Omega}_{f(t)}, \mathbb{R}\right), t \in J,
\end{aligned}
$$

and satisfying pointwise the following set of equations

$$
\begin{aligned}
\Delta u(t, z) & =0 & & t \in J, z \in \Omega_{f(t)}, \\
\partial_{y} u(t, z) & =0 & & t \in J, z \in \Gamma_{0}, \\
u(t, z) & =f(t, x) & & t \in J, z \in \Gamma_{f(t)}, \\
\lim _{|z| \rightarrow \infty} u(t, z) & =c & & t \in J, \\
\partial_{t} f(t, x)+\sqrt{1+\partial_{x} f^{2}(t, x)} \partial_{n} u(t, z) & =0 & & t \in \dot{J}, z \in \Gamma_{f(t)}, \\
f(0, x) & =f_{0}(x) & & x \in \mathbb{R} .
\end{aligned}
$$

Here we use the following notation: $z=(x, y)$ represents a gencric point in $\bar{\Omega}_{f}$. Moreover, $\Delta$ denotes the Laplace operator with respect to the Euclidean metric and $\partial_{n}$ stands for the derivative in direction of the outer unit normal $n$ at $\Gamma_{f(t)}$, i.e., $\partial_{n} u:=(\nabla u \mid n)$, where $\nabla u$ and $(\cdot \cdot)$ denotes the gradient of $u$ and the inner product in $\mathbb{R}^{2}$, respectively (again in Euclidean coordinates of course). Observe that at each point $(x, f(t, x))$ of $\Gamma_{f(t)}$ the outer unit normal $n$ is given by

$$
n(t, x)=\frac{\left(-\partial_{x} f(t, x), 1\right)}{\sqrt{1+\partial_{x} f^{2}(t, x)}}, \quad t \in J, x \in \mathbb{R} .
$$

A pair $(u, f)$ satisfying $(1.1)$ and $(P)_{f_{0}, c}$ is called a classical solution of $(P)_{f_{0}, c}$ on $J$. Problem $(P)_{f_{0}, c}$ is a standard model for the flow of an incompressible Newtonian 
fluid in a homogeneous and isotropic porous medium, see [7, pp. 297, 321]. It is used in hydrology of groundwater $[7,10,17,21]$ and for Hele-Shaw flows [10, p. $160]$.

An inherent difficulty in treating problem $(P)_{f_{0}, c}$ stems from the fact that the function $f$, determining the interface $\Gamma_{f}$, is a priori unknown. Bear and Bachmat $[7$, p. 316] describe this circumstance as follows: "We find ourselves in a kind of vicious circle: in order to solve the problem, we have to know the location of the boundary, but in order to determine the shape of the boundary, we have first to solve the problem". We will break this vicious circle by solving the nonlinear equations which are behind the inherent difficulties described above.

In order to formulate our result, let $h^{s}, s>0$, denote the little Hölder spaces, see section 3 , and put $h_{c}^{s}:=\left\{c+g ; g \in h^{s}\right\}$. Moreover, let $\alpha \in(0,1)$ be fixed. Given $f \in \widetilde{\mathfrak{A}} \cap h_{c}^{2+\alpha}(\mathbb{R})$, let $u_{f}$ denote the unique solution of

$$
\Delta u=0 \quad \text { in } \quad \Omega_{f}, \quad \partial_{y} u=0 \quad \text { on } \Gamma_{0}, \quad u=f \quad \text { on } \Gamma_{f} .
$$

Moreover, set

$$
\kappa_{f}:=\frac{f^{2}}{\left(1+f^{2}+f_{x}^{2}\right)\left(1+f_{x}^{2}\right)}
$$

and define

$$
V_{c}:=\left\{f \in \widetilde{\mathfrak{A}} \cap h_{c}^{2+\alpha}(\mathbb{R}) ; \partial_{2} u_{f}(x, f(x))<\kappa_{f}(x), x \in \mathbb{R}\right\} .
$$

Observe that $f \equiv c$ belongs to $\widetilde{\mathfrak{A}} \cap h_{c}^{2+\alpha}(\mathbb{R})$ and that $u_{c} \equiv c$. Hence $c$ lies in $V_{c}$. More precisely, it can be shown that $V_{c}$ is a open neighborhood of $c$ in $h_{c}^{2+\alpha}(\mathbb{R})$ and that $\operatorname{diam}_{2+\alpha}\left(V_{c}\right):=\sup _{g, h \in V_{c}}\|g-h\|_{2+\alpha}=\infty$, see Lemma 5.10. Suppose now that $(u, f)$ is a classical solution of $(P)_{f_{0}, c}$ on $J$ for some $f_{0} \in V_{c}$. We call $(u, f)$ a classical Hölder solution on $J$ if it possesses the additional regularity

$$
\begin{aligned}
& f \in C\left(J, V_{c}\right) \cap C^{1}\left(J, h_{c}^{1+\alpha}(\mathbb{R})\right), \\
& u(t, \cdot) \in h_{c}^{2+\alpha}\left(\bar{\Omega}_{f(t)}, \mathbb{R}\right), t \in J
\end{aligned}
$$

Finally, a solution $(u, f)$ of $(P)_{f_{0}, c}$ on $J$ is said to be maximal if there does not exist an interval $\bar{J}$ and a pair of functions $(\bar{u}, \bar{f})$ such that $J$ is a proper subinterval of $\bar{J},(\bar{u}, \bar{f}) \supset(u, f)$, and such that $(\bar{u}, \bar{f})$ is a classical solution of $(P)_{f_{0}, c}$ on $\bar{J}$.

Theorem 1. Given $f_{0} \in V_{c}$, there exist $t^{+}:=t^{+}\left(f_{0}\right)>0$ and a unique maximal classical Hölder solution $(u, f)$ of problem $(P)_{f_{0}, c}$ on $\left[0, t^{+}\right)$. Additionally, the mapping $\left[\left(t, f_{0}\right) \mapsto f\right]$ defines a local $C^{\infty}$-semiflow on $V_{c}$. If $t^{+}<\infty$ and $f$ : $\left[0, t^{+}\right) \rightarrow V_{c}$ is uniformly continuous then either

$$
\lim _{t \rightarrow t^{+}}\|f(t, \cdot)\|_{2+\alpha}=\infty \quad \text { or } \quad \lim _{t \rightarrow t^{+}} \inf _{h \in \partial V_{c}}\|f(t, \cdot)-h\|_{2+\alpha}=0 .
$$

Let us briefly sketch the main steps in the proof of Theorem 1 . In the very first stage we transform problem $(P)_{f_{0}, c}$ into a problem on a fixed domain and we then 
reduce this to a nonlinear evolution equation

$$
\partial_{t} f+\widetilde{\Phi}(f)=0, \quad f(0)=f_{0}
$$

involving only the unknown function $f$, which determines the free boundary $\Gamma_{f}$. This will be done in sections 2-4 and in Appendix C, where we in particular investigate second order elliptic boundary value problems in little Hölder spaces on the strip $\Omega=\mathbb{R} \times(0,1)$ and under general boundary conditions. The operator $[f \mapsto \widetilde{\Phi}(f)]$ appearing in (1.3) is a nonlinear, nonlocal pseudo-differential operator. In addition, $\widetilde{\Phi}$ carries a quasilinear structure. More precisely, given $g \in h^{2+\alpha}$, let $\Phi(g):=\widetilde{\Phi}(g+c)$. Then it turns out that $\Phi(g)=\Lambda(g) g$, where $\Lambda(g)$, for fixed $g$, is a linear opcrator, the so-called generalized Dirichlet-Neumann operator, cf. [12].

The next step is devoted to the proof that the linearization $\partial \Phi(g)$ of $\Phi$ is the negative generator of a strongly continuous analytic semigroup on the little Hölder space $h^{1+\alpha}$, i.e., $\partial \Phi(g) \in \mathcal{H}\left(h^{1+\alpha}\right)$, provided $g+c \in V_{c}$. In this sense the phase space $V_{c}$ should be considered as the "domain of parabolicity" of problem $(P)_{f_{0}, c}$. The main ingredients here are to associate to $\partial \Phi(g)$ a Fourier operator $A$, i.e., a pseudo-differential operator with constant coefficients and to use Mihlin-Hörmander's multiplier theorem, parameter dependent norms, and the structural condition contained in $V_{c}$ to verify that $A \in \mathcal{H}\left(h^{1+\alpha}\right)$. This step will be realized in section 5 and Appendix B, where we particularly provide explicit representations of so-called Poisson and singular Green operators, respectively.

Finally, in section 6 , we use subtle perturbation arguments and the continuity method to carry over the generation property of $-A$ to $-\partial \Phi(g)$. This result enables us to apply the theory of maximal regularity due to Da Prato and Grisvard [9] and Lunardi $[18,19,20]$, and to solve the evolution equation (1.3) in the space $h_{c}^{1+\alpha}(\mathbb{R})$.

Problem $(P)_{f_{0}, c}$ has also been investigated by H. Kawarada and H. Koshigoe [21] using the implicit function theorem of Nash-Moser. More precisely, Kawarada and Koshigoe prove, for a sufficiently smooth initial condition $f_{0}$, the existence of a local solution $(u, f)$ of $(P)_{f_{0}, c}$ in the sense that there is a positive constant $\tau$ with $f \in$ $C\left((0, \tau], H_{c}^{15}(\mathbb{R})\right) \cap C^{1}\left((0, \tau], H_{c}^{1}(\mathbb{R})\right)$ and $u(t, \cdot) \in H_{c}^{15}\left(\Omega_{f(t)}, \mathbb{R}\right), t \in(0, \tau]$. Indeed, it is assumed in [21] that $f_{0} \in H_{c}^{18}(\mathbb{R})$ and that sup $\left|f_{0}(x)-c\right|<c / 2$. Here, $H^{s}:=$ $H_{2}^{s}$ denote the Sobolev spaces of order $s \geq 0$ and again $H_{c}^{s}:=\left\{c+g ; g \in H^{s}\right\}$. Observe that Kawarada and Koshigoe's result contains a (serious) loss of regularity of the solution, which makes it impossible to extend a given local solution to a maximal solution. In this sense the results in [21] are not appropriate to investigate the long time behavior of $(P)_{f_{0}, c}$. Moreover, it should be noted that there are no assertions in [21] concerning uniqueness of solutions. In contrast, our approach guarantees the existence of a unique maximal solution of $(P)_{f_{0}, c}$ possessing optimal regularity, provided the initial condition belongs to $V_{c}$. Additionally, we get the dynamic behavior (1.2) and the semiflow property. 


\section{Transformations}

In this section we transform the original problem into a problem on a fixed domain. We give a representation of the transformed operators in the corresponding new coordinates. As a consequence, it turns out that the transformed operators will depend nonlinearly on the unknown function $f$.

In the following, $c>0$ is fixed. Define

$$
\mathfrak{A}:=\mathfrak{A}_{c}:=\left\{g \in B C^{2}(\mathbb{R}) ; \inf _{x \in \mathbb{R}}(c+g(x))>0\right\} .
$$

Given $g \in \mathfrak{A}$, let $f:=c+g \in \widetilde{\mathfrak{A}}$ and

$$
\varphi\left(x^{\prime}, y^{\prime}\right):=\varphi_{g}\left(x^{\prime}, y^{\prime}\right):=\left(x^{\prime}, 1-\frac{y^{\prime}}{c+g\left(x^{\prime}\right)}\right) \quad \text { for } \quad\left(x^{\prime}, y^{\prime}\right) \in \Omega_{f} .
$$

It is easily verified that $\varphi_{g} \in \operatorname{Diff}^{2}\left(\Omega_{f}, \Omega\right)$, i.e., $\varphi_{g}$ is a diffeomorphism of class $C^{2}$ which maps $\Omega_{f}$ onto the strip $\Omega:=\mathbb{R} \times(0,1)$. Moreover,

$$
\varphi^{-1}(x, y):=\varphi_{g}^{-1}(x, y)=(x,(1-y)(c+g(x)) \quad \text { for } \quad(x, y) \in \Omega \text {. }
$$

Let

$$
\begin{array}{lll}
\varphi_{*} u:=\varphi_{*}^{g} u:=u \circ \varphi_{g}^{-1} & \text { for } & u \in C\left(\bar{\Omega}_{f}\right), \\
\varphi^{*} v:=\varphi_{g}^{*} v:=v \circ \varphi_{g} & \text { for } & v \in C(\bar{\Omega}),
\end{array}
$$

denote the push forward and pull back operators, respectively, induced by $\varphi$. Given $g \in \mathfrak{A}$ and $v \in C^{2}(\bar{\Omega})$, we define the following transformed operators:

$$
\begin{aligned}
& \mathcal{A}(g) v:=-\varphi_{*}^{g} \Delta\left(\varphi_{g}^{*} v\right) \\
& \mathcal{B}_{i}(g) v:=\varphi_{*}^{g}\left(\gamma_{i} \nabla\left(\varphi_{g}^{*} v\right) \mid n_{i}\right), \quad i=0,1,
\end{aligned}
$$

where $\gamma_{0}$ and $\gamma_{1}$ stands for the trace operator and $n_{0}=\left(-g_{x}, 1\right)$ and $n_{1}=(0,-1)$ denote the outer normal according to $\Gamma_{f}$ and $\Gamma_{0}$, respectively.

Let $g_{0} \in \mathfrak{A}$ be given and consider the following transformed problem

$$
\begin{array}{rll}
\mathcal{A}(g) v=0 & \text { in } & J \times \Omega, \\
v=g & & \text { on } \quad J \times \Gamma_{0}, \\
\mathcal{B}_{1}(g) v=0 & & \text { on } \quad J \times \Gamma_{1}, \\
\lim _{|z| \rightarrow \infty} v(t, z)=0 & \text { on } \quad J, \\
\partial_{t} g+\mathcal{B}_{0}(g) v=0 & \text { on } \quad \dot{J} \times \Gamma_{0}, \\
g(0, \cdot)=g_{0} & \text { on } & \mathbb{R},
\end{array}
$$

where $\Gamma_{i}:=\mathbb{R} \times\{i\}, i=0,1$. A pair $(v, g)$ is called a classical solution of $(Q)_{g_{0}}$ on $J$ iff

$$
\begin{aligned}
& g \in C(J, \mathfrak{A}) \cap C^{\mathbf{1}}\left(J, B C^{1}(\mathbb{R})\right), \\
& v(t, \cdot) \in B C^{2}(\bar{\Omega}), t \in J,
\end{aligned}
$$


and $(v, g)$ satisfies $(Q)_{g_{0}}$ pointwise. It is obvious that problems $(P)_{f_{0}, c}$ and $(Q)_{g_{0}}$ are equivalent in the following sense:

Lemma 2.1. Let $g_{0} \in \mathfrak{A}$ and $c>0$ be given.

a) If $(u, f)$ is a classical solution of $(P)_{c+g_{0}, c}$ then $\left(\varphi_{*}^{f-c} u-c, f-c\right)$ is a classical solution of $(Q)_{g_{0}}$.

b) If $(v, g)$ is classical solution of $(Q)_{g_{0}}$ then $\left(\varphi_{g}^{*} v+c, g+c\right)$ is a classical solution of $(P)_{c+g_{0}, c}$.

The next Lemma gives a representation of the transformed operators $\mathcal{A}(g)$ and $\mathcal{B}(g)$ in local coordinates. Let $\pi(x, y):=1-y$ for $(x, y) \in \bar{\Omega}, h_{x}=\partial h$ for $h \in C^{1}(\mathbb{R})$, and assume that $g \in \mathfrak{A}$. We set

$$
\begin{aligned}
& a_{11}(g):=1, \quad a_{12}(g):=a_{21}(g):=\frac{\pi g_{x}}{c+g}, \quad a_{22}(g):=\frac{1}{(c+g)^{2}}\left(1+\pi^{2} g_{x}^{2}\right), \\
& a_{2}(g):=\frac{\pi}{c+g}\left(\frac{2 g_{x}^{2}}{c+g}-g_{x x}\right), \\
& b_{1,0}(g):=-g_{x}, \quad b_{2,0}(g):=-\frac{1}{c+g}\left(1+g_{x}^{2}\right), \quad b_{1,1}(g):=0, \quad b_{2,1}(g):=\frac{1}{c+g} .
\end{aligned}
$$

Lemma 2.2. Given $g \in \mathfrak{A}$, we have

$$
\mathcal{A}(g)=\sum_{j, k=1}^{2}-a_{j k}(g) \partial_{j} \partial_{k}+a_{2}(g) \partial_{2}, \quad \mathcal{B}_{i}(g)=\sum_{j=1}^{2} b_{j, i}(g) \gamma_{i} \partial_{j}, \quad i=0,1
$$

and

$$
\sum_{j, k=1}^{2} a_{j k}(g) \xi^{j} \xi^{k} \geq \underline{\alpha}(g)|\xi|^{2} \quad \text { for } \quad \xi \in \mathbb{R}^{2}
$$

where

$$
\underline{\alpha}(g):=\frac{1}{1+(c+g)^{2}+\pi^{2} g_{x}^{2}} .
$$

Proof. Let $\widetilde{g}_{j k}:=\widetilde{g}_{j k}(g):=\left(\partial_{j} \varphi_{g}^{-1} \mid \partial_{k} \varphi_{g}^{-1}\right), 1 \leq j, k \leq 2$, denote the components of the metric tensor. Then it is easily verified that $\widetilde{g}:=\operatorname{det}\left[\widetilde{g}_{j k}\right]=(c+g)^{2}$ and that

$$
\left[\widetilde{g}^{j k}\right]:=\left[\widetilde{g}_{j k}\right]^{-1}=\left(\begin{array}{cc}
1 & \frac{\pi g_{x}}{c+g} \\
\frac{\pi g_{x}}{c+g} & \frac{1}{(c+g)^{2}}\left(1+\pi^{2} g_{x}^{2}\right)
\end{array}\right) .
$$

Now the first assertion follows from the well-known formulas

$$
\begin{aligned}
& \mathcal{A}(g) v=-\varphi_{*}^{g} \Delta\left(\varphi_{g}^{*} v\right)=-\frac{1}{\sqrt{\widetilde{g}}} \sum_{j, k=1}^{2} \partial_{j}\left(\sqrt{\widetilde{g}} \widetilde{g}^{j k} \partial_{k} v\right), \\
& \mathcal{B}_{i}(g) v=\varphi_{*}^{g}\left(\gamma_{i} \nabla\left(\varphi_{g}^{*} v\right) \mid n_{i}\right)=\varphi_{*}^{g}\left(\gamma_{i}\left[\sum_{k=1}^{2} \partial_{j} \varphi_{g}^{k} \partial_{k} v\right]_{1 \leq j \leq 2} \mid n_{i}\right), \quad i=0,1 .
\end{aligned}
$$


To prove the second assertion, we fix $(x, y) \in \bar{\Omega}$ and suppress it in our notation. Observe that the smallest eigenvalue of $\left[\widetilde{g}^{j k}\right]$ is given by

$$
\lambda_{-}(g)=\frac{1}{2(c+g)^{2}}\left\{1+(c+g)^{2}+\pi^{2} g_{x}^{2}-\sqrt{\left[1+(c+g)^{2}+\pi^{2} g_{x}^{2}\right]^{2}-4(c+g)^{2}}\right\}
$$

Set

$$
\alpha:=\alpha(g):=\left[1+(c+g)^{2}+\pi^{2} g_{x}^{2}\right]^{-1}, \quad \beta:=\beta(g):=2(c+g),
$$

and note that

$$
\frac{1}{\alpha^{2}}-\beta^{2}=\left[1-(c+g)^{2}-\pi^{2} g_{x}^{2}\right]^{2}+4 \pi^{2} g_{x}^{2} \geq 0 .
$$

Hence we find that $\alpha^{2} \beta^{2} \leq 1$. This implies that

$$
0 \leq 1-\alpha^{2} \beta^{2} \leq\left(1-\frac{\alpha^{2} \beta^{2}}{2}-\right)^{2}
$$

Consequently,

$$
\lambda_{-}(g)=\frac{2}{\alpha \beta^{2}}\left(1-\sqrt{1-\alpha^{2} \beta^{2}}\right) \geq \alpha,
$$

which completes the proof of Lemma 2.2.

\section{Elliptic boundary value problems in Hölder spaces}

In this section we study linear elliptic boundary value problems on so-called little Hölder spaces $h^{\alpha}(\Omega)$. We will derive a priori estimates as well as isomorphism properties for such problems. In the following let $\mathcal{S}\left(\mathbb{R}^{n}\right), n \geq 1$, denote the Schwartz space, i.e., the Fréchet space of all rapidly decreasing smooth functions on $\mathbb{R}^{n}$. Moreover, assume that $k \in \mathbb{N}$ and that $U$ is an open subset of $\mathbb{R}^{n}$. Let

$$
B U C^{k}(U):=\left(\left\{u \in C^{k}(U) ;\|u\|_{k, U}:=\max _{|\alpha| \leq k} \sup _{x \in U}\left|\partial^{\alpha} u(x)\right|<\infty\right\} ;\|\cdot\|_{k}\right)
$$

denote the Banach space of all functions on $U$ having bounded and uniformly continuous derivatives of order $k$. The $\alpha$-Hölder seminorm, $\alpha \in(0,1)$, is defined as

$$
[u]_{\alpha, U}:=\sup _{\substack{x, y \in U \\ x \neq y}} \frac{|u(x)-u(y)|}{|x-y|^{\alpha}} .
$$

Then we define the Banach space

$$
B U C^{k+\alpha}(U):=\left(\left\{u \in B U C^{k}(U) ; \max _{|\beta|=k}\left[\partial^{\beta} u\right]_{\alpha, U}<\infty\right\} ;\|\cdot\|_{k+\alpha, U}\right),
$$


where

$$
\|u\|_{k+\alpha, U}:=\|u\|_{k, U}+\max _{|\beta|=k}\left[\partial^{\beta} u\right]_{\alpha, U}, \quad k \in \mathbb{N}, \alpha \in(0,1) .
$$

Suppose that $p, q \in[1, \infty]$ and that $s \in \mathbb{R}$ and let $B_{p q}^{s}\left(\mathbb{R}^{n}\right)$ denote the Besov spaces over $\mathbb{R}^{n}$, cf. [29, Definition 1.5.1]. The following result characterizes the spaces $B U C^{s}\left(\mathbb{R}^{n}\right)$ as Besov spaces, provided $s$ is not an integer.

Theorem 3.1. Assume that $s \in \mathbb{R}^{+} \backslash \mathbb{N}$. Then

$$
B U C^{s}\left(\mathbb{R}^{n}\right)=B_{\infty \infty}^{s}\left(\mathbb{R}^{n}\right)
$$

Proof. This follows from Theorem 1.5.1 (ii) and Theorem 1.2.2 (ii) in [29]. In order to have a consistent notation, we set

$$
B U C^{s}\left(\mathbb{R}^{n}\right):=B_{\infty \infty}^{s}\left(\mathbb{R}^{n}\right), \quad s<0 .
$$

After these preparations we now define the little Hölder spaces of order $s$, to be

$$
h^{s}\left(\mathbb{R}^{n}\right):=\text { closure of } \mathcal{S}\left(\mathbb{R}^{n}\right) \text { in } B U C^{s}\left(\mathbb{R}^{n}\right), \quad s \in \mathbb{R} .
$$

Finally, suppose that $U$ is an open subset of $\mathbb{R}^{n}$ and let $r_{U}$ denote the restriction operator with respect to $U$, i.e., $r_{U}:=u \mid U$ for $u \in B U C\left(\mathbb{R}^{n}\right)$. Given $s \geq 0$, we define

$$
h^{s}(U):=\text { closure of } r_{U}\left(\mathcal{S}\left(\mathbb{R}^{n}\right)\right) \text { in } B U C^{s}(U) .
$$

Lemma 3.2. Suppose that $M$ is an open subset of $\mathbb{R}^{n}$ which is uniformly regular of class $C^{\infty}$ in the sense of $[8$, Definition 1, p. 28]. Then

a) $h^{t}(M) \stackrel{d}{\leftrightarrow} h^{s}(M), \quad 0 \leq s<t$.

b) There exists an extension operator $\mathcal{E} \in \mathcal{L}\left(h^{s}(M), h^{s}\left(\mathbb{R}^{n}\right)\right), s \geq 0$, such that $r_{M} \mathcal{E}=i d_{h^{s}(M)}$.

Proof. a) is obvious.

b) Fix $s \geq 0$. Using Theorem 3.1, Theorem 4.5.2 (ii) in [29], Lemma 11.2 in [2], and the fact that $M$ is uniformly regular of class $C^{\infty}$, it follows, by means of local coordinates, that there exist $s_{1} \in \mathbb{N}$ with $s_{1}>s$ and an extension opcrator $\mathcal{E}$ such that

$$
\mathcal{E} \in \mathcal{L}\left(B U C^{r}(M), B U C^{r}\left(\mathbb{R}^{n}\right)\right) \quad \text { with } \quad r_{M} \mathcal{E}=\operatorname{id}_{B U C^{r}(M)}, \quad r \in\left[0, s_{1}\right] .
$$

In particular we have

$$
\mathcal{E} \in \mathcal{L}\left(h^{s}(M), B U C^{s}\left(\mathbb{R}^{n}\right)\right) \quad \text { with } \quad r_{M} \mathcal{E}=\mathrm{id}_{h^{s}(M)}
$$


Thus it suffices to show that

$$
\mathcal{E} u \in h^{s}\left(\mathbb{R}^{n}\right) \quad \text { for } \quad u \in h^{s}(M) .
$$

There exists a sequence $u_{k} \in \mathcal{S}\left(\mathbb{R}^{n}\right)$ such that $r_{M} u_{k} \rightarrow u$ in $B U C^{s}(M)$ as $k \rightarrow \infty$. Thus (3.6) implies that $\mathcal{E} r_{M} u_{k} \in B U C^{s_{1}}\left(\mathbb{R}^{n}\right)$. Since $s_{1} \in \mathbb{N}$, it is known that

$$
h^{s_{1}}\left(\mathbb{R}^{n}\right)=\left\{u \in C^{s_{1}}\left(\mathbb{R}^{n}\right) ; \partial^{\alpha} u \in C_{0}\left(\mathbb{R}^{n}\right), \quad|\alpha| \leq s_{1}\right\},
$$

where $C_{0}\left(\mathbb{R}^{n}\right)$ denotes the space of all continuous functions on $\mathbb{R}^{n}$ vanishing at infinity. Now the construction of $\mathcal{E}$ and the fact that $u_{k} \in \mathcal{S}\left(\mathbb{R}^{n}\right)$ yield that $\mathcal{E} r_{M} u_{k} \in h^{s_{1}}\left(\mathbb{R}^{n}\right)$. But $\mathcal{E} r_{M} u_{k} \rightarrow \mathcal{E} u$ in $B U C^{s}\left(\mathbb{R}^{n}\right)$ as $k \rightarrow \infty$. Therefore we see that $\mathcal{E} u \in h^{s}\left(\mathbb{R}^{n}\right)$.

Remarks 3.3. a) Given $0<s<t$, it is known that $B U C^{t}\left(\mathbb{R}^{n}\right)$ is not dense in $B U C^{s}\left(\mathbb{R}^{n}\right)$. For that reason we prefer to work in little Hölder spaces, rather then in the classical Hölder spaces $B U C^{s}$.

b) Suppose that $\alpha \in(0,1)$ and that $k \in \mathbb{N}$. Then the following characterization of the little Hölder spaces holds: $u \in h^{k+\alpha}\left(\mathbb{R}^{n}\right)$ iff $u \in C_{0}^{k}\left(\mathbb{R}^{n}\right)$ and

$$
\lim _{t \rightarrow 0} \sup _{\substack{x, y \in \mathbb{R}^{n} \\ 0<|x-y| \leq t}} \frac{\left|\partial^{\beta} u(x)-\partial^{\beta} u(y)\right|}{|x-y|^{\alpha}}=0, \quad \beta \in \mathbb{N}^{n},|\beta|=k .
$$

A proof in the case $n=1$ is given in [26]. For the general case see [4].

We need some further function spaces. Let $U$ be an open subset of $\mathbb{R}^{n}$ and assume that $s \geq 0$. We set

$$
b u c^{s}(U):=\text { closure of } B U C^{\infty}(U) \text { in } B U C^{s}(U) .
$$

Moreover, we use the following notations:

$$
h^{s}:=h^{s}(\mathbb{R}), \quad h_{\mathfrak{A}}^{s}:=h^{s} \cap \mathfrak{A}, \quad \text { buc }^{s}:=b u c^{s}(\mathbb{R}), \quad s \geq 0 .
$$

Given $s \geq 0$, it is easily verified that

$$
\begin{aligned}
& {[(a, v) \mapsto a v]: b u c^{s} \times h^{s}(\Omega) \rightarrow h^{s}(\Omega),} \\
& {[(a, g) \mapsto a g]: b u c^{s} \times h^{s} \rightarrow h^{s}}
\end{aligned}
$$

are bilinear and continuous, and that the mapping

$$
\left[g \mapsto \frac{1}{c+g}\right]: h_{\mathfrak{A}}^{s} \rightarrow b u c^{s}
$$

is of class $C^{\infty}$. 
Lemma 3.4. Let $\alpha \in(0,1)$ be given. Then

$$
\left(\mathcal{A}(\cdot), \mathcal{B}_{0}(\cdot)\right) \in C^{\infty}\left(h_{\mathfrak{A}}^{2+\alpha}, \mathcal{L}\left(h^{2+\alpha}(\Omega), h^{\alpha}(\Omega) \times h^{1+\alpha}\right)\right)
$$

and

$$
\begin{gathered}
\partial \mathcal{A}(g)[h, v]=\frac{2 \pi}{c+g}\left\{\left(\frac{g_{x} h}{c+g}-h_{x}\right) \partial_{1} \partial_{2} v+\left(\frac{h}{(c+g)^{2}}\left(\frac{1}{\pi}+\pi g_{x}^{2}\right)-\frac{\pi}{c+g} g_{x} h_{x}\right) \partial_{2}^{2} v\right. \\
\left.-\left(\frac{g_{x}^{2} h}{(c+g)^{2}}-\frac{g_{x x} h+4 g_{x} h_{x}}{2(c+g)}+\frac{h_{x x}}{2}\right) \partial_{2} v\right\}, \\
\partial \mathcal{B}_{0}(g)[h, v]=-h_{x} \partial_{1} v+\frac{1}{c+g}\left(\frac{h+h g_{x}^{2}}{c+g}-2 g_{x} h_{x}\right) \partial_{2} v,
\end{gathered}
$$

for $h \in h^{2+\alpha}, v \in h^{2+\alpha}(\Omega)$.

Proof. This follows from (3.8), (3.9), Lemma 2.2, and elementary calculations.

In the following we let $\alpha \in(0,1)$ be fixed. The next lemma collects some fundamental a priori estimates and isomorphism properties of elliptic boundary value problems on little Hölder spaces. A proof is given in Appendix C. It uses the classical results of Agmon, Douglis, and Nirenberg, the maximum principle, and the continuity method.

Theorem 3.5. a) Let $g \in h_{\mathfrak{A}}^{2+\alpha}, \lambda \geq 0$, and $\mu>0$ be given. Then

$$
\begin{aligned}
& \left(\lambda+\mathcal{A}(g), \gamma_{0},(c+g) \mathcal{B}_{1}(g)\right) \in \operatorname{Isom}\left(h^{2+\alpha}(\Omega), h^{\alpha}(\Omega) \times h^{2+\alpha} \times h^{1+\alpha}\right) \\
& \left(\lambda+\mathcal{A}(g), \mu \gamma_{0}+\mathcal{B}_{0}(g), \mathcal{B}_{1}(g)\right) \in \operatorname{Isom}\left(h^{2+\alpha}(\Omega), h^{\alpha}(\Omega) \times h^{1+\alpha} \times h^{1+\alpha}\right)
\end{aligned}
$$

b) Given $\lambda_{0} \geq 0$ and $g \in h_{\Omega}^{2+\alpha}$, there exists a positive constant $C$, depending on $\|g\|_{h^{2+\alpha}}, \lambda_{0}, \alpha$, and $c$, such that

$$
\|u\|_{2+\alpha, \Omega} \leq C\left(\|(\lambda+\mathcal{A}(g)) u\|_{\alpha, \Omega}+\left\|\gamma_{0} u\right\|_{2+\alpha}+\left\|(c+g) \mathcal{B}_{1}(g) u\right\|_{1+\alpha}\right)
$$

for all $u \in h^{2+\alpha}(\Omega)$ and $\lambda \in\left[0, \lambda_{0}\right]$.

Proof. See Appendix C.

Given $g \in h_{\mathfrak{A}}^{2+\alpha}$, we now define

$$
\begin{aligned}
& \mathcal{R}(g):=\left(\mathcal{A}(g), \gamma_{0},(c+g) \mathcal{B}_{1}(g)\right)^{-1}, \\
& \mathcal{S}(g):=\mathcal{R}(g) \mid h^{\alpha}(\Omega) \times\{0\} \times\{0\} \\
& \mathcal{T}(g):=\mathcal{R}(g) \mid\{0\} \times h^{2+\alpha} \times\{0\} .
\end{aligned}
$$

Assume that $g \in h_{\mathfrak{2}}^{2+\alpha}, h \in h^{2+\alpha}$, and put $u:=\mathcal{T}(g) h$. Then $u$ is the unique solution in $h^{2+\alpha}(\Omega)$ of the following elliptic boundary value problem

$$
\mathcal{A}(g) u=0 \quad \text { in } \quad \Omega, \quad \gamma_{0} u=h \quad \text { on } \quad \Gamma_{0}, \quad \mathcal{B}_{1}(g) u=0 \quad \text { on } \Gamma_{1} \text {. }
$$


Lemma 3.6. We have

$$
\mathcal{T}(\cdot) \in C^{\infty}\left(h_{\mathfrak{\mathfrak { A }}}^{2+\alpha}, \mathcal{L}\left(h^{2+\alpha}, h^{2+\alpha}(\Omega)\right)\right)
$$

and

$$
\partial \mathcal{T}(g)[h, \cdot]=-\mathcal{S}(g) \partial \mathcal{A}(g)[h, \mathcal{T}(g) \cdot]
$$

for $g \in h_{\mathfrak{A}}^{2+\alpha}$ and $h \in h^{2+\alpha}$.

Proof. To shorten our notation, let $U:=h_{\mathfrak{A}}^{2+\alpha}, F_{0}:=h^{\alpha}(\Omega), F_{2}:=h^{2+\alpha}(\Omega)$, $E_{1}:=h^{1+\alpha}$, and $E_{2}:=h^{2+\alpha}$.

a) Observe that $U$ is an open subset of $E_{2}$. Moreover, letting

$$
A(g):=\left(\mathcal{A}(g), \gamma_{0},(c+g) \mathcal{B}_{1}(g)\right), \quad g \in U,
$$

it follows from Lemma 3.4 and Theorem 3.5 that

$$
A \in C^{\infty}\left(U, \operatorname{Isom}\left(F_{2}, F_{0} \times E_{2} \times E_{1}\right)\right)
$$

with (note that $(c+g) \mathcal{B}_{1}(g)$ is independent of $g$ )

$$
\partial A(g) h=(\partial \mathcal{A}(g)[h, \cdot], 0,0) \quad \text { for } \quad h \in E_{2} .
$$

b) Given $A \in \operatorname{Isom}\left(F_{2}, F_{0} \times E_{2} \times E_{1}\right)$, define $j(A):=A^{-1}$. Then $\operatorname{Isom}\left(F_{2}, F_{0} \times\right.$ $\left.E_{2} \times E_{1}\right)$ is open in $\mathcal{L}\left(F_{2}, F_{0} \times E_{2} \times E_{1}\right)$, and it is known that

$$
j \in C^{\infty}\left(\operatorname{Isom}\left(F_{2}, F_{0} \times E_{2} \times E_{1}\right), \mathcal{L}\left(F_{0} \times E_{2} \times E_{1}, F_{2}\right)\right)
$$

with

$$
\partial j(A) \widetilde{A}=-A^{-1} \widetilde{A} A^{-1}
$$

for $A \in \operatorname{Isom}\left(F_{2}, F_{0} \times E_{2} \times E_{1}\right)$ and $\left.\tilde{A} \in \mathcal{L}\left(F_{2}, F_{0} \times E_{2} \times E_{1}\right)\right)$.

c) Let $\left.R \in \mathcal{L}\left(F_{0} \times E_{2} \times E_{1}, F_{2}\right)\right)$ be given, and define $p(R) \in \mathcal{L}\left(E_{2}, F_{2}\right)$ by

$$
p(R) x_{2}:=R\left(0, x_{2}, 0\right) \text { for } x_{2} \in E_{2} .
$$

Then $p \in \mathcal{L}\left(\mathcal{L}\left(F_{0} \times E_{2} \times E_{1}, F_{2}\right), \mathcal{L}\left(E_{2}, F_{2}\right)\right)$ and consequently

$$
\partial p(R) S=p(S) \quad \text { for } \quad R, S \in \mathcal{L}\left(F_{0} \times E_{2} \times E_{1}, F_{2}\right) .
$$

Now the assertion follows from the identity $\mathcal{T}=p \circ j \circ A$ and the chain rule.

\section{The nonlinear operator}

In this section we introduce the basic nonlinear operator and we derive some first properties of it. Moreover, we show that the corresponding evolution problem involving this operator is equivalent to the original problem $(P)_{g_{0}, c}$. Given $g \in$ $h_{\mathfrak{A}}^{2+\alpha}$, we define

$$
\Phi(g):=\mathcal{B}_{0}(g) \mathcal{T}(g) g .
$$


Observe that Lemma 3.4 and Lemma 3.6 yield that

$$
\Phi \in C^{\infty}\left(h_{\mathfrak{A}}^{2+\alpha}, h^{1+\alpha}\right) .
$$

Suppose that $g_{0} \in h_{\mathfrak{\Omega}}^{2+\alpha}, T>0$, and let $J:=[0, T)$. A function $g: J \rightarrow h^{1+\alpha}$ is said to be a classical solution of

$$
\dot{g}+\Phi(g)=0, \quad g(0)=g_{0},
$$

iff $g \in C\left(J, h_{\mathfrak{A}}^{2+\alpha}\right) \cap C^{1}\left(J, h^{1+\alpha}\right)$ and $g$ satisfies $(E)_{g_{0}}$ pointwise.

Lemma 4.1. Let $g_{0} \in h_{\mathfrak{A}}^{2+\alpha}$ be given.

a) Suppose that $g$ is a classical solution of problem $(E)_{g_{0}}$ on $J$. Let $v(t, \cdot):=$ $\mathcal{T}(g(t)) g(t)$. Then the pair $(v, g)$ is a classical solution of $(Q)_{g_{0}}$ on $J$, having the additional regularity

$$
\begin{aligned}
& g \in C\left(J, h_{\mathfrak{A}}^{2+\alpha}\right) \cap C^{1}\left(J, h^{1+\alpha}\right), \\
& v(t, \cdot) \in h^{2+\alpha}(\Omega), \quad t \in J
\end{aligned}
$$

b) Suppose that $(v, g)$ is a classical solution of $(Q)_{g_{0}}$ on $J$, having the regularity $(R)_{a}$. Then $g$ is a classical solution of $(E)_{g_{0}}$ on $J$.

Proof. Just use the definitions.

Remarks 4.2. a) Observe that for fixed $g \in h_{\mathfrak{A}}^{2+\alpha}$, we have the linear operator

$$
\Lambda(g):=\left[h \mapsto \mathcal{B}_{0}(g) \mathcal{T}(g) h\right] \in \mathcal{L}\left(h^{2+\alpha}, h^{1+\alpha}\right) .
$$

Thus problem $(E)_{g_{0}}$ is in fact a quasilinear evolution equation. The linear operator $\Lambda(g)$ is sometimes called generalized Dirichlet-Neumann operator, see [12].

b) However, it is important to note that $\Lambda$ only maps an open subset $W$ of $h^{2+\alpha}$ into $\mathcal{L}\left(h^{2+\alpha}, h^{1+\alpha}\right)$ but not an open subset $\widetilde{W}$ of some (true) intermediate space between $h^{2+\alpha}$ and $h^{1+\alpha}$ into $\mathcal{L}\left(h^{2+\alpha}, h^{1+\alpha}\right)$. If the latter were the case we could treat $(E)_{g_{0}}$ by using the general theory for abstract quasilinear parabolic equations developed by Amann in $[3,4]$, or the results in $[6,24]$. In fact, it would then sufficc to prove that $\Lambda(g) \in \mathcal{H}\left(h^{2+\alpha}, h^{1+\alpha}\right)$ for $g \in \widetilde{W}$, see also Remark 4.4 below.

This lack of a "regularizing effect" forces us to really consider $(E)_{g_{0}}$ as a fully nonlinear evolution equation. At this point we will use maximal regularity results due to Da Prato and Grisvard.

Lemma 4.3. $\Phi \in C^{\infty}\left(h_{\mathfrak{A}}^{2+\alpha}, h^{1+\alpha}\right)$ and

$$
\partial \Phi(g) h=\mathcal{B}_{0}(g) \mathcal{T}(g) h+\partial \mathcal{B}_{0}(g)[h, \mathcal{T}(g) g]-\mathcal{B}_{0}(g) \mathcal{S}(g) \partial \mathcal{A}(g)[h, \mathcal{T}(g) g]
$$

for $g \in h_{\mathfrak{X}}^{2+\alpha}$ and $h \in h^{2+\alpha}$.

Proof. This follows from Lemmas 3.4 and 3.6 . 
Remark 4.4. Let $g \in h_{\mathfrak{A}}^{2+\alpha}$ be fixed. As already mentioned in the introduction, our main effort will be concentrated on the proof that $\partial \Phi(g) \in \mathcal{H}\left(h^{2+\alpha}, h^{1+\alpha}\right)$. It is known that the Dirichlet-Neumann operator $\Lambda(g)$ generates strongly continuous analytic semigroups on various function spaces, including Besov spaces, Lebesgue spaces, and the space of continuous functions, see $[5,11,12,16]$. We shall extend these results in Section 6 by proving that $\Lambda(g) \in \mathcal{H}\left(h^{2+\alpha}, h^{1+\alpha}\right)$ for $g \in h_{\mathfrak{A}}^{2+\alpha}$. Hence Lemma 4.3 shows that $\partial \Phi(g)$ can be considered as a perturbation of the generator $-\Lambda(g)$ by the linear operators

$$
\begin{aligned}
\Theta(g) & :=\left[h \mapsto \partial \mathcal{B}_{0}(g)[h, \mathcal{T}(g) g]\right] \\
\Sigma(g) & :=\left[h \mapsto-\mathcal{B}_{0}(g) \mathcal{S}(g) \partial \mathcal{A}(g)[h, \mathcal{T}(g) g]\right]
\end{aligned}
$$

Observe that $\Theta(g), \Sigma(g) \in \mathcal{L}\left(h^{2+\alpha}, h^{1+\alpha}\right)$ and that Lemmas 2.2, 3.4 and Theorem $3.5 b)$ imply that there is a positive constant $C$, independent of $g$, such that

$$
\|\Theta(g)+\Sigma(g)\|_{\mathcal{L}\left(h^{2+\alpha}, h^{1+\alpha}\right)} \leq C\|g\|_{2+\alpha}
$$

for all $g \in h_{\mathfrak{A}}^{2+\alpha}$ with $\|g\|_{2+\alpha} \leq \sigma$, where $\sigma \in(0, c)$ is fixed. Hence, using wellknown perturbation results for the class $\mathcal{H}\left(h^{2+\alpha}, h^{1+\alpha}\right)$, see Theorem I.1.3.1 in [4], we find that $\partial \Phi(g) \in \mathcal{H}\left(h^{2+\alpha}, h^{1+\alpha}\right)$, provided $g$ is small enough, say $\|g\|_{2+\alpha} \leq \varepsilon$ for some $\varepsilon \in(0, \sigma]$. However, this result is a purely local statement in the sense that one has no information about the size of $\varepsilon$. In contrast, we propose a different approach to prove that $-\partial \Phi(g), g \in W$, generates a strongly continuous analytic semigroup on $h^{1+\alpha}$ for a large class $W$. This result enables us to construct maximal solutions of the original problem $(P)_{g_{0}, c}$ on a phase space $W$ possessing an explicit description. In particular, it turns out that $W$ is large in the sense that its diameter (in $h^{2+\alpha}$ ) is unbounded.

\section{Fourier operators of the linearization}

In this section we associate to each part $\Lambda, \Theta$, and $\Sigma$ (see (4.2) and (4.3)) of the linearization $\partial \Phi$ a Fourier operator, i.e., a pseudo differential operator with constant coefficients.

Throughout this section, let $g \in h_{\mathfrak{A}}^{2+\alpha}, x_{0} \in \mathbb{R}$, and $\mu_{0}>0$ be fixed. Define

$$
\begin{aligned}
& a_{12}:=a_{12}\left(g, x_{0}\right):=a_{12}(g)\left(x_{0}, 0\right), \quad a_{22}:=a_{22}\left(g, x_{0}\right):=a_{22}(g)\left(x_{0}, 0\right), \\
& b_{1}:=b_{1}\left(g, x_{0}\right):=b_{1,0}(g)\left(x_{0}\right), \quad b_{2}:=b_{2}\left(g, x_{0}\right):=b_{2,0}(g)\left(x_{0}\right) .
\end{aligned}
$$

We use the notation of Appendix B. Observe that due to Lemma 2.2, assumption $(B .3)$ is satisfied with $\alpha_{0}:=\underline{\alpha}(g)\left(x_{0}, 0\right)$ and that $\alpha_{*}=\frac{\sqrt{\alpha_{0}}}{a_{22}}>0$, see Remark B.1. Moreover, recall that

$$
\mathcal{A}_{\pi} u=-\partial_{1}^{2} u-2 a_{12} \partial_{1} \partial_{2} u-a_{22} \partial_{2}^{2} u
$$


and let

$$
\mathcal{B}_{\pi} u:=b_{1} \partial_{1} u+b_{2} \partial_{2} u
$$

for $u \in B U C^{2}\left(\mathbb{H}^{2}\right)$.

I. The principal part of $\Lambda(g)$. Define

$$
A_{\pi, 1}:=\mathcal{B}_{\pi} \mathcal{I}_{\pi} \quad \text { and } \quad a_{1}(\eta, \mu):=i b_{1} \eta-b_{2} \lambda(\eta, \mu),(\eta, \mu) \in \mathbb{R} \times(0, \infty),
$$

where $\mathcal{T}_{\pi}:=\mathcal{T}_{\pi, \mu_{0}}$, see $(B .5)$. For the definition of $\lambda(\eta, \mu)$ see Remark B.1b). Note that the operator $A_{\pi, 1}=\mathcal{B}_{\pi} \mathcal{T}_{\pi}$ can be considered as the principal part of $\Lambda(g)=\mathcal{B}_{0}(g) \mathcal{T}(g)$ with constant coefficients fixed in $\left(x_{0}, 0\right)$. Let us start with the following

Lemma 5.1. $a_{1} \in \mathcal{E} l l \mathcal{S}_{1}^{\infty}\left(\alpha_{*}\right)$ and $A_{\pi, 1}$ is the Fourier operator with symbol $a_{1}\left(\cdot, \mu_{0}\right)$, i.e.,

$$
A_{\pi, 1}=\mathcal{F}^{-1} a_{1}\left(\cdot, \mu_{0}\right) \mathcal{F} \text {. }
$$

Proof. We know from Remark B.1c) that $\lambda \in \mathcal{E l l S}_{1}^{\infty}\left(\alpha_{*}\right)$. Moreover, observe that $b_{2}<0$, cf. Lemma 2.2. Hence $a_{1} \in \mathcal{E} l l \mathcal{S}_{1}^{\infty}\left(\alpha_{*}\right)$, and Theorem A.2 yields that

$$
\mathcal{F}^{-1} a_{1}\left(\cdot, \mu_{0}\right) \mathcal{F} \in \mathcal{L}\left(h^{2+\alpha}, h^{1+\alpha}\right) .
$$

Given $f \in \mathcal{S}$, it is easily verified that

$$
\begin{aligned}
& \partial_{1} \mathcal{I}_{\pi} f(x, y)=\mathcal{F}^{-1}\left(\left[\eta \mapsto i \eta e^{-\lambda\left(\eta, \mu_{0}\right) y}\right] \mathcal{F} f\right)(x), \\
& \partial_{2} \mathcal{T}_{\pi} f(x, y)=-\mathcal{F}^{-1}\left(\lambda\left(\cdot, \mu_{0}\right) e^{-\lambda\left(\cdot, \mu_{0}\right) y} \mathcal{F} f\right)(x)
\end{aligned}
$$

for $(x, y) \in \mathbb{H}^{2}$, and consequently

$$
A_{\pi, 1} f=\mathcal{F}^{-1} a_{1}\left(\cdot, \mu_{0}\right) \mathcal{F} f \text { for } f \in \mathcal{S} \text {. }
$$

Since $\mathcal{S}$ is dense in $h^{2+\alpha}$, the assertions follow from (5.4) and the fact that $A_{\pi, 1} \in$ $\mathcal{L}\left(h^{2+\alpha}, h^{1+\alpha}\right)$, see Lemma B.2.

II. The principal part of $\Theta(g)$. Let

$$
\begin{gathered}
v:=v_{g}:=\mathcal{T}(g) g \in h^{2+\alpha}(\Omega), \\
c_{\pi}:=-\partial_{1} v_{g}\left(x_{0}, 0\right)-\frac{2 g_{x}\left(x_{0}\right)}{c+g\left(x_{0}\right)} \partial_{2} v_{g}\left(x_{0}, 0\right),
\end{gathered}
$$

and define

$$
A_{\pi, 2} h:=c_{\pi} \partial h, \quad h \in h^{1}, \quad a_{2}(\eta):=i c_{\pi} \eta, \quad \eta \in \mathbb{R} .
$$

Then $A_{\pi, 2}=\mathcal{F}^{-1} a_{2} \mathcal{F}$ can be considered as the principal part of $\Theta(g)=\partial \mathcal{B}_{0}(g)\left[\cdot, v_{g}\right]$ with constant coefficients fixed in $\left(x_{0}, 0\right)$. 
III. The principal part of $\Sigma(g)$. Let $v_{g}$ as in (5.5) and set

$$
w(x, y):=w_{g}(x, y):=-\frac{\pi(x, y)}{c+g(x)} \partial_{2} v_{g}(x, y), \quad(x, y) \in \Omega
$$

and

$$
w_{\pi}:=w\left(x_{0}, 0\right) .
$$

Furthermore, given $h \in h^{2+\alpha}$, define

$$
\left(\mathcal{P}_{\pi} h\right)(x, y):=\left(\mathcal{P}_{\pi}\left(g, x_{0}\right) h\right)(x, y):=w_{\pi} \partial^{2} h(x) e^{-y}, \quad(x, y) \in \mathbb{H}^{2}
$$

For later purposes we need the following technical

Lemma 5.2. a) $\mathcal{P}_{\pi} \in \mathcal{L}\left(h^{2+\alpha}, h^{\alpha}\left(\mathbb{H}^{2}\right)\right)$.

b) There exists a positive constant $C:=C(g)$ such that

$$
\left\|\mathcal{P}_{\pi} h-w \partial^{2} h\right\|_{0, \mathbb{H}^{2} \cap r \mathbb{R}\left(x_{0}, 0\right)}+r^{\alpha}\left[\mathcal{P}_{\pi} h-w \partial^{2} h\right]_{\alpha, \mathbb{H}^{2} \cap r \mathbb{B}\left(x_{0}, 0\right)} \leq C r\|h\|_{2+\alpha}
$$

for all $h \in h^{2+\alpha}$ and all $r \in(0,1]$.

Proof. a) Given $h \in h^{2+\alpha}$, there is a sequence $\left(h_{n}\right) \subset \mathcal{S}$ such that $h_{n} \rightarrow h$ in $B U C^{2+\alpha}$ as $n \rightarrow \infty$. Let

$$
w_{n}(x, y):=w_{\pi} \partial^{2} h_{n}(x) e^{-\sqrt{\left(y^{2}+\frac{1}{n}\right)}} \text { for }(x, y) \in \mathbb{R}^{2}, n \in \mathbb{N} .
$$

Then $w_{n} \in \mathcal{S}\left(\mathbb{R}^{2}\right)$ and $r_{\mathbb{H}^{2}} w_{n} \rightarrow \mathcal{P}_{\pi} h$ in $B U C^{\alpha}\left(\mathbb{H}^{2}\right)$ as $n \rightarrow \infty$, i.e., $\mathcal{P}_{\pi} h \in h^{\alpha}\left(\mathbb{H}^{2}\right)$. Since obviously $\mathcal{P}_{\pi} \in \mathcal{L}\left(B U C^{2+\alpha}, B U C^{\alpha}\left(\mathbb{H}^{2}\right)\right)$, the proof of a) is complete.

b) Recall that $w_{\pi}=w\left(x_{0}, 0\right)$ and that $w \in B U C^{1}\left(\mathbb{H}^{2}\right)$. Hence letting $U:=\mathbb{H}^{2} \cap$ $r \mathbb{B}\left(x_{0}, 0\right)$, the mean value theorem implies the existence of a positive constant $C:=C(g)$ such that

$$
\left\|\mathcal{P}_{\pi} h-w \partial^{2} h\right\|_{0, U}=\left\|\left(w_{\pi} e^{-y}-w\right) \partial^{2} h\right\|_{0, U} \leq C r\|h\|_{2+\alpha}, \quad r \in(0,1],
$$

and such that

$$
\begin{aligned}
{\left[\mathcal{P}_{\pi} h-w \partial^{2} h\right]_{\alpha, U} } & =\left[\left(w_{\pi} e^{-y}-w\right) \partial^{2} h\right]_{\alpha, U} \\
& \leq\left\|w_{\pi} e^{-y}-w\right\|_{0, U}\left[\partial^{2} h\right]_{\alpha, U}+\left[w_{\pi} e^{-y}-w\right]_{\alpha, U}\left\|\partial^{2} h\right\|_{0, U} \\
& \leq C\|w\|_{1, \Omega}\|h\|_{2+\alpha}\left(r+r^{1-\alpha}\right), \quad r \in(0,1]
\end{aligned}
$$

This proves assertion $b$ ).

In the following let

$$
\widetilde{w}(y):=w_{\pi} e^{-|y|} \quad \text { for } \quad y \in \mathbb{R}
$$

and observe that

$$
\mathcal{F} \widetilde{w}(\theta)=\sqrt{\frac{2}{\pi}} \frac{w_{\pi}}{1+\theta^{2}}, \quad \theta \in \mathbb{R}
$$


cf. (B.32). Morcover, due to (B.3), we have that

$$
\mu_{0}^{2}+p(\eta, \theta) \geq \mu_{0}^{2}+\alpha_{0}\left(\eta^{2}+\theta^{2}\right) \geq 2 \sqrt{\alpha_{0}}\left(\mu_{0}^{2}+\alpha_{0} \eta^{2}\right)^{\frac{1}{2}} \theta
$$

Hence, putting

$$
a_{3,1}(\eta, \mu):=\frac{i b_{2}}{\sqrt{2 \pi}} \eta^{2} \int_{\mathbb{R}} \frac{\theta \mathcal{F} \widetilde{w}(\theta)}{\mu^{2}+p(\eta, \theta)} d \theta \text { for }(\eta, \mu) \in \mathbb{R} \times(0, \infty),
$$

Lebesgue's Theorem implies that $a_{3,1}\left(\cdot, \mu_{0}\right) \in C^{1}(\mathbb{R})$ with

$$
\partial_{1} a_{3,1}\left(\eta, \mu_{0}\right)=\frac{2 i b_{2} \eta}{\sqrt{2 \pi}} \int_{\mathbb{R}} \theta \mathcal{F} \tilde{w}(\theta) \frac{\mu_{0}^{2}+a_{12} \eta \theta+a_{22} \theta^{2}}{\left[\mu_{0}^{2}+p(\eta, \theta)\right]^{2}} d \theta
$$

Let us also introduce the following symbol

$$
j_{1}(\eta):=\sqrt{1+\eta^{2}} \text { for } \eta \in \mathbb{R}
$$

It is known that

$$
\mathcal{F}^{-1} j_{1}^{\sigma} \mathcal{F} \in \operatorname{Isom}\left(h^{s+\sigma}, h^{s}\right) \quad \text { with } \quad\left[j_{1}^{\sigma}\right]^{-1}=j_{1}^{-\sigma} \text { for } s, \sigma \in \mathbb{R} \text {. }
$$

Assume that $\beta \in(0, \alpha)$. Then, using (5.7)-(5.9), elementary calculations show that

$$
j_{1}^{\alpha-\beta-1} a_{3,1}\left(\cdot, \mu_{0}\right) \in \mathcal{M}
$$

Let

$$
A_{\pi, 3,1}:=-b_{2} \gamma \partial_{2} \mathcal{S}_{\pi, 1} \mathcal{P}_{\pi}
$$

where $\mathcal{S}_{\pi, 1}:=\mathcal{S}_{\pi, \mu_{0}, 1}$, see $(B .27)$. From Lemma 5.2a) and Lemma. B.9 we know that $A_{\pi, 3,1} \in \mathcal{L}\left(h^{2+\alpha}, h^{1+\alpha}\right)$. The following Lemma shows that this result can be improved.

Lemma 5.3. Assume that $\beta \in(0, \alpha)$. Then $A_{\pi, 3,1}$ has an extension, again denoted by $A_{\pi, 3,1}$, such that

$$
A_{\pi, 3,1} \in \mathcal{L}\left(h^{2+\beta}, h^{1+\alpha}\right)
$$

and

$$
A_{\pi, 3,1}=\mathcal{F}^{-1} a_{3,1}\left(\cdot, \mu_{0}\right) \mathcal{F}
$$

Proof. Given $h \in \mathcal{S}$, we have

$$
\left[\mathcal{F}_{2} \mathcal{E} \mathcal{P}_{\pi} h\right](\eta, \theta)=-\eta^{2} \mathcal{F} h(\eta) \cdot \mathcal{F} \widetilde{w}(\theta), \quad(\eta, \theta) \in \mathbb{R}^{2}
$$


where $\mathcal{E}$ is given in $(B .23)$. Consequently, it follows that

$$
\begin{aligned}
-b_{2} \gamma \partial_{2} \mathcal{S}_{\pi, 1} \mathcal{P}_{\pi} h & =-b_{2} \gamma \partial_{2} r_{\mathbb{H}^{2}} \mathcal{F}_{2}^{-1}\left(\mu_{0}^{2}+p\right)^{-1} \mathcal{F}_{2} \mathcal{E} \mathcal{P}_{\pi} h \\
& =-b_{2} \gamma r_{\mathbb{H}^{2}} \mathcal{F}_{2}^{-1}\left[(\eta, \theta) \mapsto i \theta\left(\mu_{0}^{2}+p(\eta, \theta)\right)^{-1}\right] \mathcal{F}_{2} \mathcal{E \mathcal { P } _ { \pi } h} \\
& =-\frac{b_{2}}{2 \pi}\left[x \mapsto \int_{\mathbb{R}} e^{i x \eta} \int_{\mathbb{R}} \frac{i \theta}{\mu_{0}^{2}+p(\eta, \theta)} \mathcal{F}_{2} \mathcal{E} \mathcal{P}_{\pi} h(\eta, \theta) d \theta d \eta\right] \\
& =\mathcal{F}^{-1} a_{3,1}\left(\cdot, \mu_{0}\right) \mathcal{F} h
\end{aligned}
$$

for $h \in \mathcal{S}$. Moreover, we conclude from (5.10), (5.11), and Theorem A.1 that

$$
\mathcal{F}^{-1} a_{3,1}\left(\cdot, \mu_{0}\right) \mathcal{F}=\mathcal{F}^{-1} j_{1}^{\alpha-\beta-1} a_{3,1}\left(\cdot, \mu_{0}\right) \mathcal{F} \mathcal{F}^{-1} j_{1}^{1+\beta-\alpha} \mathcal{F} \in \mathcal{L}\left(h^{2+\beta}, h^{1+\alpha}\right) .
$$

Now the density of $\mathcal{S}$ in $h^{2+\beta}$ implies the assertions.

We introduce a further operator

$$
A_{\pi, 3,2}:=-b_{2} \gamma \partial_{2} \mathcal{S}_{\pi, 2} \mathcal{P}_{\pi}
$$

where $\mathcal{S}_{\pi, 2}=\mathcal{S}_{\pi, \mu_{0}, 2}$, see $(B .27)$, and a further symbol

$$
a_{3,2}(\eta, \mu)=\frac{b_{2} w_{\pi}}{a_{22}} \eta^{2} \frac{\lambda(\eta, \mu)}{d(\eta, \mu)} \frac{d(\eta, \mu)+1}{|\lambda(\eta, \mu)+1|^{2}}, \quad(\eta, \mu) \in \mathbb{R} \times(0, \infty) .
$$

Lemma 5.4. $A_{\pi, 3,2} \in \mathcal{L}\left(h^{2+\alpha}, h^{1+\alpha}\right)$ and

$$
A_{\pi, 3,2}=\mathcal{F}^{-1} a_{3,2}\left(\cdot, \mu_{0}\right) \mathcal{F} \text {. }
$$

Proof. The first assertion follows again from Lemma 5.2a) and Lemma B.8. Given $h \in \mathcal{S}$, we have

$$
\mathcal{F} \mathcal{P}_{\pi} h(\eta, y)=-w_{\pi} \eta^{2} e^{-y} \mathcal{F} h(\eta) \quad \text { for } \quad(\eta, y) \in \mathbb{H}^{2},
$$

see the definition preceding Remark B.6. Hence, it follows that

$$
\begin{aligned}
k_{\mathcal{P}_{\pi} h, \mu_{0}}(\eta) & =-\frac{1}{a_{22}} \int_{0}^{\infty} e^{-d\left(\eta, \mu_{0}\right) y} \cos (a(\eta) y) \mathcal{F} \mathcal{P}_{\pi} h(\eta, y) d y \\
& =\frac{w_{\pi}}{a_{22}} \eta^{2} \mathcal{F} h(\eta) \int_{0}^{\infty} e^{-d\left(\eta, \mu_{0}\right) y} \cos (a(\eta) y) e^{-y} d y \\
& =\frac{w_{\pi}}{2 a_{22}} \eta^{2} \mathcal{F} h(\eta) \int_{0}^{\infty} e^{-d\left(\eta, \mu_{0}\right) y}\left[e^{i a(\eta) y}+e^{-i a(\eta) y}\right] e^{-y} d y \\
& =\frac{w_{\pi}}{a_{22}} \eta^{2} \mathcal{F} h(\eta) \frac{d\left(\eta, \mu_{0}\right)+1}{\left|\lambda\left(\eta, \mu_{0}\right)+1\right|^{2}} .
\end{aligned}
$$

Consequently, the definition of $\mathcal{S}_{\pi, 2}$ implies

$$
A_{\pi, 3,2}=-b_{2} \gamma \partial_{2} \mathcal{F}^{-1} \frac{e^{-\lambda\left(\cdot, \mu_{0}\right) y}}{d\left(\cdot, \mu_{0}\right)} k_{\mathcal{P}_{\pi} h, \mu_{0}}(\cdot)=\mathcal{F}^{-1} a_{3,2}\left(\cdot, \mu_{0}\right) \mathcal{F}
$$

This proves the Lemma. 
Let $A_{\pi, 3}:=-\mathcal{B}_{\pi} \mathcal{S}_{\pi} \mathcal{P}_{\pi}$, where $\mathcal{S}_{\pi}:=\mathcal{S}_{\pi, \mu_{0}}=\mathcal{S}_{\pi, 1}+\mathcal{S}_{\pi, 2}$. Then we have

Corollary 5.5. $A_{\pi, 3} \in \mathcal{L}\left(h^{2+\alpha}, h^{1+\alpha}\right)$ and $A_{\pi, 3}$ is represented by the Fourier operator with symbol $a_{3,1}\left(\cdot, \mu_{0}\right)+a_{3,2}\left(\cdot, \mu_{0}\right)$.

Proof. Observe that $\gamma \partial_{1} u=\partial \gamma u$ for $u \in h^{2+\alpha}\left(\mathbb{H}^{2}\right)$ and that $\gamma \mathcal{S}_{\pi}=0$. Hence $\gamma \partial_{1} \mathcal{S}_{\pi} \mathcal{P}_{\pi}=0$ and therefore

$$
\begin{aligned}
A_{\pi, 3} & =-\mathcal{B}_{\pi} \mathcal{S}_{\pi} \mathcal{P}_{\pi}=-b_{1} \gamma \partial_{1} \mathcal{S}_{\pi} \mathcal{P}_{\pi}-b_{2} \gamma \partial_{2} \mathcal{S}_{\pi} \mathcal{P}_{\pi} \\
& =A_{\pi, 3,1}+A_{\pi, 3,2}=\mathcal{F}^{-1}\left(a_{3,1}\left(\cdot, \mu_{0}\right)+a_{3,2}\left(\cdot, \mu_{0}\right)\right) \mathcal{F}
\end{aligned}
$$

It follows from the definition of $\mathcal{P}_{\pi}$ and the representation of $\partial \mathcal{A}$, see Lemma 3.4, that the operator $A_{\pi, 3}$ can be considered as the principal part of $\Sigma(g)=$ $-\mathcal{B}_{0}(g) \mathcal{S}(g) \partial \mathcal{A}(g)\left[\cdot, v_{g}\right]$ with coefficients fixed in $\left(x_{0}, 0\right)$. Hence, summarizing the above results, the Fourier operator

$$
A_{\pi}:=A_{\pi, 1}+A_{\pi, 2}+A_{\pi, 3}
$$

may be viewed as the principal part of the linearization $\partial \Phi(g)$ with coefficients fixed in $\left(x_{0}, 0\right)$. Our next goal is to prove that $A_{\pi}$ belongs to the class $\mathcal{H}\left(h^{2+\alpha}, h^{1+\alpha}\right)$. In order to do this, set

$$
a_{\pi, t}(\eta, \mu):=\left(b_{1}+t c_{\pi}\right) i \eta-b_{2} \lambda(\eta, \mu)\left\{1-t \frac{w_{\pi}}{a_{22}} \eta^{2} \frac{\mu_{0}^{2} d(\eta, \mu)+\mu_{0} \mu}{d(\eta, \mu)\left|\mu_{0} \lambda(\eta, \mu)+\mu\right|^{2}}\right\}
$$

for $t \in[0,1]$ and $(\eta, \mu) \in \mathbb{R} \times(0, \infty)$. Observe that $a_{\pi, 0}\left(\cdot, \mu_{0}\right)$ and $a_{\pi, 1}\left(\cdot, \mu_{0}\right)$ are the symbols of $\mathcal{B}_{\pi} \mathcal{T}_{\pi}$ and $A_{\pi, 1}+A_{\pi, 2}+A_{\pi, 3,2}$, respectively. Our main result of this section reads as follows:

Theorem 5.6. Suppose that

$$
w_{\pi}<\frac{\alpha_{0}}{a_{22}} .
$$

Then there exists an $\alpha_{\pi}>0$ such that

$$
a_{\pi, t} \in \mathcal{E} l l \mathcal{S}_{1}^{\infty}\left(\alpha_{\pi}\right) \text { for all } t \in[0,1] .
$$

Before proving Theorem 5.6, let us write down

Corollary 5.7. If condition (5.15) holds then $A_{\pi} \in \mathcal{H}\left(h^{2+\alpha}, h^{1+\alpha}\right)$.

Proof. Combining Theorem 5.6 (in the case $t=1, \mu=\mu_{0}$ ) and Theorem A.2, it follows that

$$
A_{\pi, 1}+A_{\pi, 2}+A_{\pi, 3,2} \in \mathcal{H}\left(h^{2+\alpha}, h^{1+\alpha}\right) .
$$

Fix $\beta \in(0, \alpha)$. Then $h^{2+\beta}=\left(h^{1+\alpha}, h^{2+\alpha}\right)_{1+\beta-\alpha, \infty}^{0}$, where $(\cdot, \cdot)_{\theta, \infty}^{0}, \theta \in(0,1)$, denotes the continuous interpolation method of Da Prato and Grisvard, see $[9,6$, 
20, 24]. Together with Lemma 5.3 we find for each $\varepsilon>0$ positive constants $C$ and $C_{1}(\varepsilon)$ such that

$$
\left\|A_{\pi, 3,1} h\right\|_{1+\alpha} \leq C\|h\|_{2+\beta} \leq \varepsilon\|h\|_{2+\alpha}+C_{1}(\varepsilon)\|h\|_{1+\alpha}
$$

for all $h \in h^{2+\alpha}$. Hence Theorem I.1.3.1 in [4], a perturbation result for the class $\mathcal{H}\left(h^{2+\alpha}, h^{1+\alpha}\right)$, implies that

$$
A_{\pi}=A_{\pi, 1}+A_{\pi, 2}+A_{\pi, 3,2}+A_{\pi, 3,1} \in \mathcal{H}\left(h^{2+\alpha}, h^{1+\alpha}\right) .
$$

Let us also add the following

Remark 5.8. Condition (5.15) depends on $g \in h_{\mathfrak{A}}^{2+\alpha}$ and on $x_{0} \in \mathbb{R}$, but is independent of $\mu_{0}$. More precisely, (5.15) reads as

$$
w_{\pi}=w_{g}\left(x_{0}, 0\right)<\frac{\alpha(g)\left(x_{0}, 0\right)}{a_{22}(g)\left(x_{0}, 0\right)}=\frac{\alpha_{0}}{a_{22}} .
$$

Proof of Theorem 5.6. Fix $t \in[0,1]$. Obviously, $a_{\pi, t} \in C^{\infty}(\mathbb{R} \times(0, \infty), \mathbb{C})$ and $a_{\pi, t}$ is positively homogeneous of degree 1 . It remains to show that there exists an $\alpha_{\pi}$ such that

$$
\operatorname{Re} a_{\pi, t}(\eta, \mu) \geq \alpha_{\pi} \sqrt{\eta^{2}+\mu^{2}} \quad \text { for } \quad(\eta, \mu) \in \mathbb{R} \times(0, \infty) .
$$

Pick $(\eta, \mu) \in \mathbb{R} \times(0, \infty)$ and observe that

$$
\operatorname{Re} a_{\pi, t}(\eta, \mu)=-b_{2} d(\eta, \mu)\left\{1-t \frac{w_{\pi}}{a_{22}} \eta^{2} \frac{\mu_{0}^{2} d(\eta, \mu)+\mu_{0} \mu}{d(\eta, \mu)\left|\mu_{0} \lambda(\eta, \mu)+\mu\right|^{2}}\right\}
$$

Further, Remark B.1c) shows that

$$
d(\eta, \mu) \geq \frac{\sqrt{\alpha_{0}}}{a_{22}} \sqrt{\eta^{2}+\mu^{2}} \geq \frac{\sqrt{\alpha_{0}}}{a_{22}}|\eta|
$$

i) Suppose that $w_{\pi} \leq 0$. Then it follows from (5.17) and $b_{2}<0$ that (5.16) is satisfied with $\alpha_{\pi}:=-b_{2} \frac{\sqrt{\alpha_{0}}}{a_{22}}$.

ii) Suppose now that $w_{\pi} \in\left(0, \frac{\alpha_{0}}{a_{22}}\right)$ and put $\delta:=\frac{\alpha_{0}}{a_{22}}-w_{\pi}>0$. It is not difficult to see that

$$
\frac{\mu_{0}^{2} d(\eta, \mu)+\mu_{0} \mu}{d(\eta, \mu)\left|\mu_{0} \lambda(\eta, \mu)+\mu\right|^{2}} \leq \frac{1}{[d(\eta, \mu)]^{2}} \leq \frac{a_{22}^{2}}{\alpha_{0} \eta^{2}}
$$

where we used (5.17) to obtain the second inequality. Since $-b_{2}$ and $w_{\pi}$ are both positive, we conclude that

$$
\operatorname{Re} a_{\pi, t}(\eta, \mu) \geq-b_{2} d(\eta, \mu)\left\{1-t w_{\pi} \frac{a_{22}}{\alpha_{0}}\right\} \geq-b_{2} \frac{a_{22}}{\alpha_{0}} \delta d(\eta, \mu)
$$

Using once again (5.17), we find that (5.16) is satisfied with $\alpha_{\pi}:=-\frac{b_{2} \delta}{\sqrt{\alpha_{0}}}$. This completes the proof. 
Remark 5.9. The proof of Theorem 5.6 shows the following sharper result: Let $t \in[0,1]$ be given and assume that

$$
t w_{\pi}<\frac{\alpha_{0}}{a_{22}}
$$

Then there is an $\alpha_{\pi, t}>0$ such that $a_{\pi, t} \in \mathcal{E} l l \mathcal{S}_{1}^{\infty}\left(\alpha_{\pi, t}\right)>0$.

In particular, we see that condition (5.18) is always satisfied in case $t=0$.

As already observed in Remark 5.8, condition (5.15) depends on $g \in h_{\mathfrak{A}}^{2+\alpha}$ and on $x \in \mathbb{R}$. We now specify a subset $W_{1}$ of $h_{\mathfrak{A}}^{2+\alpha}$ such that for fixed $g \in W_{1}$ condition (5.15) is satisfied for all $x \in \mathbb{R}$. Recall that

$$
\underline{\alpha}(g)=\frac{1}{1+(c+g)^{2}+\pi^{2} g_{x}^{2}}
$$

and

$$
a_{22}(g)=\frac{1}{(c+g)^{2}}\left(1+\pi^{2} g_{x}^{2}\right)
$$

for $g \in h_{\mathfrak{Q}}^{2+\alpha}$. Moreover, define

$$
\kappa_{g}(x):=\frac{\underline{\alpha}(g)(x)}{a_{22}(g)(x, 0)}=\frac{(c+g(x))^{2}}{\left(1+(c+g(x))^{2}+g_{x}^{2}(x)\right)\left(1+g_{x}^{2}(x)\right)}, \quad x \in \mathbb{R} .
$$

Note that $\inf _{x \in \mathbb{R}} \kappa_{g}(x)>0$ for $g \in h_{\mathfrak{A}}^{2+\alpha}$. We now introduce

$$
W_{t}:=W_{\alpha, t}:=\left\{g \in h_{\mathfrak{A}}^{2+\alpha} ; \inf _{x \in \mathbb{R}}\left(\frac{t}{c+g(x)} \partial_{2} v_{g}(x, 0)+\kappa_{g}(x)\right)>0\right\}, \quad t \in[0,1],
$$

where $v_{g}=\mathcal{T}_{0}(g) g$, cf. (5.5). Obviously, $W_{t} \subset W_{s}$ for $0 \leq s \leq t \leq 1$ and $W_{0}=$ $h_{\mathfrak{a}}^{2+\alpha}$. Finally, given a subset $X \subset h^{2+\alpha}$, let

$$
\operatorname{diam}_{2+\alpha}(X):=\sup _{g, h \in X}\|g-h\|_{2+\alpha}
$$

denote the diameter of $X$ in $h^{2+\alpha}$. Then we have the following

Lemma 5.10. Let $t \in[0,1]$ be given. Then $W_{t}$ is an open neighborhood of 0 in $h_{\mathfrak{A}}^{2+\alpha}$ and $\operatorname{diam}_{2+\alpha}\left(W_{t}\right)=\infty$.

Proof. Fix $t \in[0,1]$. Obviously, $0 \in h_{\mathfrak{A}}^{2+\alpha}$. Moreover, $\kappa_{0}=\frac{c^{2}}{1+c^{2}}$ and $v_{0}=0$. Hence 0 belongs to $W_{t}$. Given $g \in h_{\mathfrak{a}}^{2+\alpha}$, put (see also (5.6))

$$
w_{g, t}(x):=-t w_{g}(x, 0)=\frac{t}{c+g(x)} \partial_{2} v_{g}(x, 0), x \in \mathbb{R} .
$$

Then it follows from the a priori estimates in Theorem 3.5 that

$$
\left[g \mapsto w_{g, t}\right] \in C\left(h_{\mathfrak{A}}^{2+\alpha}, B U C\right) .
$$


Moreover, observe that

$$
\left[g \mapsto \kappa_{g}\right] \in C\left(h_{\mathfrak{A}}^{2+\alpha}, B U C\right)
$$

Thus, letting

$$
B_{t}(g):=\inf _{x \in \mathbb{R}}\left(w_{g, t}(x)+\kappa_{g}(x)\right) \text { for } g \in h_{\mathfrak{a}}^{2+\alpha}
$$

it follows that

$$
B_{t} \in C\left(h_{\mathfrak{A}}^{2+\alpha}, \mathbb{R}\right) .
$$

Consequently, $W_{t}=B_{t}^{-1}((0, \infty))$ is open in $h_{\mathfrak{A}}^{2+\alpha}$.

It remains to prove that $\operatorname{diam}_{2+\alpha}\left(W_{t}\right)=\infty$. For this fix $\beta \in(0, \alpha)$ and $\sigma \in(0, c)$.

From Theorem $3.5 \mathrm{~b}$ ) we know that there is a constant $M>0$ such that

$$
\|\mathcal{T}(g)\|_{\mathcal{L}\left(h^{2+\beta}, h^{2+\beta}(\Omega)\right)} \leq M
$$

for all $g \in h_{\mathfrak{A}}^{2+\beta}$ satisfying $\|g\|_{2+\beta} \leq \sigma$. Now define

$$
\kappa:=\frac{(c-\sigma)^{3}}{2 M\left(1+(c+\sigma)^{2}+\sigma^{2}\right)\left(1+\sigma^{2}\right)} \wedge \sigma
$$

and choose a sequence $\left(g_{n}\right)_{n \in \mathbb{N}} \subset h^{2+\alpha}$ such that

$$
\sup _{n \in \mathbb{N}}\left\|g_{n}\right\|_{2+\beta}<\kappa, \quad \sup _{n \in \mathbb{N}}\left\|g_{n}\right\|_{2+\alpha}=\infty
$$

Observe that $\left\|g_{n}\right\|_{\infty}<\sigma<c$ particularly implies that $\left(g_{n}\right)_{n \in \mathbb{N}} \subset h_{\mathfrak{A}}^{2+\alpha}$. Moreover it follows from (5.19) that

$$
\left|\left(g_{n}\right)_{x}(x)\right|^{2} \leq\left\|g_{n}\right\|_{2+\beta}^{2}<\sigma^{2}, \quad n \in \mathbb{N}, \quad x \in \mathbb{R}
$$

and therefore

$$
\kappa_{g_{n}}(x) \geq \frac{(c-\sigma)^{2}}{\left(1+(c+\sigma)^{2}+\sigma^{2}\right)\left(1+\sigma^{2}\right)}, \quad n \in \mathbb{N}, \quad x \in \mathbb{R} .
$$

But we also have

$$
\begin{aligned}
-\frac{1}{c+g_{n}(x)} \partial_{2} v_{g_{n}}(x, 0) & \leq \frac{1}{c-\sigma}\left\|v_{g_{n}}\right\|_{2+\beta, \Omega}=\frac{1}{c-\sigma}\left\|\mathcal{T}\left(g_{n}\right) g_{n}\right\|_{2+\beta, \Omega} \\
& \leq \frac{M}{c-\sigma}\left\|g_{n}\right\|_{2+\beta} \leq \frac{1}{2} \cdot \frac{(c-\sigma)^{2}}{\left(1+(c+\sigma)^{2}+\sigma^{2}\right)\left(1+\sigma^{2}\right)}
\end{aligned}
$$

for $n \in \mathbb{N}, x \in \mathbb{R}$. Combining (5.20) and that last inequality, we find that $\left(g_{n}\right)_{n \in \mathbb{N}} \subset$ $W_{1} \subset W_{t}$. Since $\sup _{n \in \mathbb{N}}\left\|g_{n}\right\|_{2+\alpha}=\infty$ we conclude that diam $\operatorname{dit\alpha }_{2+\alpha}\left(W_{t}\right)$ is $\infty$.

Remark 5.11. Suppose that $g \in W_{1}$. Then

$$
w_{\pi}=w_{g}(x, 0)<\kappa_{g}(x)=\frac{\underline{\alpha}(g)(x, 0)}{a_{22}(g)(x, 0)}=\frac{\alpha_{0}}{a_{22}}
$$

for all $x \in \mathbb{R}$, see also (5.15). 


\section{A priori estimates for the linearization}

In this section we use the Fourier operator $A_{\pi}$ of the preceding section to derive Hölder a priori estimates for the linearization $\partial \Phi$. Essentially, this will be obtained by estimating $\theta \partial \Phi-A_{\pi} \theta$, where $\theta$ is a smooth function with small support. Throughout this section $\mu_{0}>0$ is fixed. Moreover, we put $W:=W_{1}$. Let $a_{\pi, t}$ denote the symbol of (5.14) and define

$$
A_{\pi, t}:=\mathcal{F}^{-1}\left[a_{\pi, t}\left(\cdot, \mu_{0}\right)+t a_{\pi, 3,1}\left(\cdot, \mu_{0}\right)\right] \mathcal{F} \quad \text { for } \quad t \in[0,1] .
$$

Given $g \in h_{\mathfrak{A}}^{2+\alpha}$ and $t \in[0,1]$, set

$$
\partial \Phi_{t}(g):=\mathcal{B}_{0}(g) \mathcal{T}(g)+t \partial \mathcal{B}_{0}(g)[\cdot, \mathcal{T}(g) g]-t \mathcal{B}_{0}(g) \mathcal{S}(g) \partial \mathcal{A}(g)[\cdot, \mathcal{T}(g) g]
$$

and observe that $\partial \Phi_{0}(g)=\mathcal{B}_{0}(g) \mathcal{T}(g)=\Lambda(g)$ and $\partial \Phi_{1}(g)=\partial \Phi(g)$. Hence the parameter $t$ deforms the Dirichlet-Neumann operator $\Lambda(g)$ into the linearization $\partial \Phi(g)$.

Let $\rho>0$ be given and let $\left\{\left(U_{j}, \theta_{j}\right) ; j \in \mathbb{N}\right\}$ denote a $\rho$-localization sequence for $S:=S_{\rho}:=\mathbb{R} \times(-\rho / 2, \rho / 2)$, i.e., each $U_{j}$ is open in $S, \bigcup_{j \in \mathbb{N}} U_{j}=S$, the covering $\left\{U_{j} ; j \in \mathbb{N}\right\}$ has finite multiplicity, $\operatorname{diam} U_{j} \leq \rho$, and $\left(U_{j}, \theta_{j}^{2}\right)$ is a partition of unity on $S$. Moreover, we fix $x_{j} \in \mathbb{R}$ such that $\left(x_{j}, 0\right) \in U_{j}, j \in \mathbb{N}$. Our main technical tool is the following

Lemma 6.1. Assume that $K \subset W$ is compact, $\beta \in(0, \alpha)$, and that $\kappa>0$. Then there exist $\rho \in(0,1]$, a $\rho$-localization sequence $\left\{\left(U_{j}, \theta_{j}\right) ; j \in \mathbb{N}\right\}$, and a positive constant $C:=C(K, \kappa, \rho)$ such that

$$
\left\|\left[\theta_{j} \partial \Phi_{t}(g)-A_{\pi, t}\left(g, x_{j}\right) \theta_{j}\right] h\right\|_{1+\alpha} \leq \kappa\left\|\theta_{j} h\right\|_{2+\alpha}+C\|h\|_{2+\beta}
$$

for all $h \in h^{2+\alpha}, j \in \mathbb{N}, t \in[0,1]$, and $g \in K$.

Before proving that Lemma, let us give the following application of it:

Theorem 6.2. Assume that $K \subset W$ is compact. Then there exist positive constants $\mu_{*}$ and $C:=C(K)$ such that

$$
\|h\|_{2+\alpha}+|\mu|\|h\|_{1+\alpha} \leq C\left\|\left(\mu+\partial \Phi_{t}(g)\right) h\right\|_{1+\alpha}
$$

for all $h \in h^{2+\alpha}, g \in K, t \in[0,1]$, and $\mu \in\left[\operatorname{Re} z>\mu_{*}\right]$.

Proof. Let $K \subset W$ be compact, $g \in K$, and $j \in \mathbb{N}$. From Remark 5.11, Theorem 5.6 , a perturbation argument for $t A_{\pi, 3,1}$ similar as in the proof of Corollary 5.7, and Theorem A.2 we infer that there exist positive constants $\widetilde{C}$ and $\widetilde{\mu}_{*}$ such that

$$
\|h\|_{2+\alpha}+|\mu|\|h\|_{1+\alpha} \leq \widetilde{C}\left\|\left(\mu+A_{\pi, t}\left(g, x_{j}\right)\right) h\right\|_{1+\alpha}
$$


for all $h \in h^{2+\alpha}, g \in K, j \in \mathbb{N}, t \in[0,1]$, and $\mu \in\left[\operatorname{Re} z>\widetilde{\mu}_{*}\right]$. Fix $\beta \in(0, \alpha)$. Due to Lemma 6.1 there exists a $\rho$-localization sequence $\left\{\left(U_{j}, \theta_{j}\right) ; j \in \mathbb{N}\right\}$ and a positive constant $C$ such that

$$
\left\|\left[\theta_{j} \partial \Phi_{t}(g)-A_{\pi, t}\left(g, x_{j}\right) \theta_{j}\right] h\right\|_{1+\alpha} \leq \frac{1}{2 \widetilde{C}}\left\|\theta_{j} h\right\|_{2+\alpha}+C\|h\|_{2+\beta}
$$

for all $h \in h^{2+\alpha}, j \in \mathbb{N}, t \in[0,1]$, and $g \in K$. Therefore, replacing $h$ by $\theta_{j} h$ in (6.1), we find that

$$
\left\|\theta_{j} h\right\|_{2+\alpha}+|\mu|\left\|\theta_{j} h\right\|_{1+\alpha} \leq 2 \widetilde{C}\left\{\left\|\theta_{j}\left(\mu+\partial \Phi_{t}(g)\right) h\right\|_{1+\alpha}+C\|h\|_{2+\beta}\right\}
$$

for all $h \in h^{2+\alpha}, g \in K, t \in[0,1]$, and $\mu \in\left[\operatorname{Re} z>2 \widetilde{\mu}_{*}\right]$. Since $\left\{\left(U_{j}, \theta_{j}\right) ; j \in \mathbb{N}\right\}$ is a localization sequence for $S$, it can be shown that

$$
\left[h \mapsto \sup _{j \in \mathbb{N}}\left\|\theta_{j} h\right\|_{k+\alpha}\right]
$$

defines an equivalent norm on $h^{k+\alpha}, k=1,2$. Hence there is a positive constant $C$ such that

$$
\|h\|_{2+\alpha}+|\mu|\|h\|_{1+\alpha} \leq \frac{C}{2}\left\|\left(\mu+\partial \Phi_{t}(g)\right) h\right\|_{1+\alpha}+C\|h\|_{2+\beta}
$$

for all $h \in h^{2+\alpha}, g \in K, t \in[0,1]$, and $\mu \in\left[\operatorname{Re} z>2 \widetilde{\mu}_{*}\right]$. Finally, we have the interpolation result

$$
h^{2+\beta}=\left(h^{1+\alpha}, h^{2+\alpha}\right)_{1-\alpha+\beta, \infty}^{0},
$$

where $(\cdot, \cdot)_{s, \infty}^{0}, s \in(0,1)$, denotes the continuous interpolation functor of Da Prato and Grisvard, see [9]. Hence there is a constant $C_{1}>0$ such that

$$
\|h\|_{2+\beta} \leq \frac{1}{2 C}\|h\|_{2+\alpha}+C_{1}\|h\|_{1+\alpha}, \quad h \in h^{2+\alpha} .
$$

Now put $\mu_{*}:=2 \widetilde{\mu}_{*} \vee 2 C_{1} C$ to complete the proof.

It is now easy to establish the following generation result for the linearization $\partial \Phi$.

Corollary 6.3. Let $g \in W$ and $t \in[0,1]$ be given. Then

$$
\partial \Phi_{t}(g) \in \mathcal{H}\left(h^{2+\alpha}, h^{1+\alpha}\right) .
$$

Proof. Pick $g \in W$. We first prove that

$$
\mu_{*}+\Lambda(g) \in \operatorname{Isom}\left(h^{2+\alpha}, h^{1+\alpha}\right),
$$

where $\mu_{*}$ has the same meaning as in Theorem 6.2. Also recall that $\Lambda(g)=$ $\mathcal{B}_{0}(g) \mathcal{T}(g)=\partial \Phi_{0}$. In view of Theorem 6.2 it suffices to verify that $\mu_{*}+\Lambda(g)$ is surjective. Hence, let $h \in h^{1+\alpha}$ be given. Due to Theorem 3.5a), we find $u \in h^{2+\alpha}(\Omega)$ such that

$$
\left(\mathcal{A}(g), \mu_{*} \gamma_{0}+\mathcal{B}_{0}(g),(c+g) \mathcal{B}_{1}(g)\right) u=(0, h, 0) .
$$


This implies that

$$
\begin{aligned}
\mathcal{T}(g) \gamma_{0} u & =\left(\mathcal{A}(g), \gamma_{0},(c+g) \mathcal{B}_{1}(g)\right)^{-1}\left(0, \gamma_{0} u, 0\right) \\
& =\left(\mathcal{A}(g), \gamma_{0},(c+g) \mathcal{B}_{1}(g)\right)^{-1}\left(\mathcal{A}(g), \gamma_{0},(c+g) \mathcal{B}_{1}(g)\right) u=u
\end{aligned}
$$

Putting $z:=\gamma_{0} u \in h^{2+\alpha}$, it follows that

$$
\left(\mu_{*}+\mathcal{B}_{0}(g) \mathcal{T}(g)\right) z=\left(\mu_{*} \gamma_{0}+\mathcal{B}_{0}(g)\right) u=h
$$

This shows (6.5). Now, due to Theorem 6.2, we can apply Theorem 5.2 in [14] to $\partial \Phi_{t}(g)$ and we find that

$$
\mu_{*}+\partial \Phi_{t}(g) \in \operatorname{Isom}\left(h^{2+\alpha}, h^{1+\alpha}\right)
$$

for all $t \in[0,1]$. It remains to combine (6.6), Theorem 6.2, and Remark 1.1.2.1a) in [4] to complete the proof of Corollary 6.3.

Remark 6.4. A precise inspection of the proofs of Theorem 6.2 and Corollary 6.3 gives the following result:

$$
\partial \Phi_{t}(g) \in \mathcal{H}\left(h^{2+\alpha}, h^{1+\alpha}\right) \text { for } t \in[0,1] \text { and } g \in W_{t}
$$

Particularly, in case $t=0$ we have for the Dirichlet-Neumann operator:

$$
\Lambda(g) \in \mathcal{H}\left(h^{2+\alpha}, h^{1+\alpha}\right) \quad \text { for all } \quad g \in h_{\mathfrak{A}}^{2+\alpha}=W_{0} .
$$

For the proof of Lemma 6.1 we need some preparation. Let $\left\{\left(U_{j}, \theta\right) ; j \in \mathbb{N}\right\}$ be a $\rho$-localization sequence for $S$. Given $j \in \mathbb{N}$, we choose $\chi_{j} \in \mathcal{D}\left(U_{j}\right)$ such that $\chi_{j} \mid \operatorname{supp} \theta_{j}=1$. We call $\left\{\left(U_{j}, \theta_{j}, \chi_{j}\right) ; j \in \mathbb{N}\right\}$ an extended $\rho$-localization sequence for $S$. Observe that the cut-off functions $\chi_{j}$ can be chosen such that there is a positive constant $C$, independent of $\rho$, with

$$
\left\|\chi_{j}\right\|_{0, U_{j}}+\rho^{\alpha}\left[\chi_{j}\right]_{\alpha, U_{j}} \leq C, \quad j \in \mathbb{N} .
$$

Moreover, there is a $Z:=Z_{\rho}>0$ such that

$$
\left\|\chi_{j}\right\|_{2+\alpha, U_{j}}+\left\|\theta_{j}\right\|_{2+\alpha, U_{j}} \leq Z, \quad j \in \mathbb{N} .
$$

Extending and restricting a test function $\chi \in \mathcal{D}\left(U_{j}\right)$, we obtain pointwise multipliers on the spaces $h^{k+\alpha}(\Omega)$ and $h^{k+\alpha}$, respectively. We use for all these multiplication operators the same symbol $\chi$. Analogously, the commutators $[A, \chi]:=$ $A \chi-\chi A$, where $A \in \mathcal{L}\left(h^{l+\alpha}(\Omega), h^{k+\alpha}\right)$ or $A \in \mathcal{L}\left(h^{l+\alpha}, h^{k+\alpha}(\Omega)\right), l \geq k$, have to be understood in this way. Given $g \in h_{\mathfrak{A}}^{2+\alpha}$, let us introduce the following linear operator

$$
\mathcal{P}:=\mathcal{P}(g):=\partial \mathcal{A}(g)\left[\cdot, v_{g}\right] \in \mathcal{L}\left(h^{2+\alpha}, h^{\alpha}(\Omega)\right)
$$


where $v_{g}=\mathcal{T}(g) g$, see (5.5). In order to further economize our notation, we fix $g \in h_{\mathfrak{A}}^{2+\alpha}, t \in[0,1], j \in \mathbb{N}$ and suppress them whenever no confusion seems likely. For example,

$$
\begin{aligned}
& \theta \partial \Phi=\theta_{j} \partial \Phi_{t}(g), \quad A_{\pi} \chi=A_{\pi, t}\left(g, x_{j}\right) \chi_{j}, \quad \text { or } \\
& \mathcal{B}_{\pi} \mathcal{S}_{\pi} \mathcal{P}_{\pi}-\mathcal{B S P}=\mathcal{B}_{\pi}\left(g, x_{j}\right) \mathcal{S}_{\pi, \mu_{0}}\left(g, x_{j}\right) \mathcal{P}_{\pi}\left(g, x_{j}\right)-\mathcal{B}_{0}(g) \mathcal{S}(g) \mathcal{P}(g),
\end{aligned}
$$

where $x_{j} \in \mathbb{R}$ with $\left(x_{j}, 0\right) \in U_{j}, j \in \mathbb{N}$, is fixed.

Moreover, recall that (see (3.10) and Corollary B.12)

$$
\begin{aligned}
& (\mathcal{A}, \gamma) \mathcal{S}=\left(i d_{h^{\alpha}(\Omega)}, 0\right), \quad\left(\mu_{0}^{2}+\mathcal{A}_{\pi}, \gamma\right) \mathcal{S}_{\pi}=\left(i d_{h^{\alpha}\left(\mathbb{H}^{2}\right)}, 0\right) \\
& (\mathcal{A}, \gamma) \mathcal{T}=\left(0, i d_{h^{2+\alpha}}\right), \quad\left(\mu_{0}^{2}+\mathcal{A}_{\pi}, \gamma\right) \mathcal{T}_{\pi}=\left(0, i d_{h^{2+\alpha}}\right) .
\end{aligned}
$$

We now return to estimate $\theta \partial \Phi-A_{\pi} \theta$. Observe that $\chi \theta=1$. Hence we have

$$
\theta \partial \Phi-A_{\pi} \theta=\chi[\theta, \partial \Phi]+\left[\chi \partial \Phi-A_{\pi} \chi\right] \theta .
$$

We first focus our considerations on the commutator $[\theta, \partial \Phi]$. In order to do this, consider the following situation: Suppose that $E_{0}, E_{\alpha}$, and $E_{1}$ are Banach spaces such that $E_{1} \hookrightarrow E_{\alpha}$. Define

$$
\mathcal{L}_{E_{1}}^{e}\left(E_{\alpha}, E_{0}\right):=\left\{A \in \mathcal{L}\left(E_{1}, E_{0}\right) ; \exists c>0:\|A x\|_{E_{0}} \leq c\|x\|_{\alpha}, x \in E_{1}\right\} .
$$

If in addition $E_{1}$ is dense in $E_{\alpha}$, then, given $A \in \mathcal{L}_{E_{1}}^{e}\left(E_{\alpha}, E_{0}\right)$, it is well-known that there is a unique extension $A^{e} \in \mathcal{L}\left(E_{\alpha}, E_{0}\right)$ of $A$. Furthermore, we use the notation $\mathcal{L}_{E_{1}}^{e}\left(E_{0}\right):=\mathcal{L}_{E_{1}}^{e}\left(E_{0}, E_{0}\right)$.

The following Lemma collects estimates for some commutators which we need for later purposes. In the sequel, let $B_{Z}:=\left\{\theta \in C^{\infty}\left(\mathbb{R}^{2}\right) ;\|\theta\|_{2+\alpha, \mathbb{R}^{2}} \leq Z\right\}$. Given $\theta \in C^{\infty}\left(\mathbb{R}^{2}\right)$, we put $\widetilde{\theta}(x, y):=\theta(x, 0)$ for $(x, y) \in \mathbb{R}^{2}$. Finally, we assume that $K \subset h_{\mathfrak{A}}^{2+\alpha}$ is compact.

Lemma 6.5. a) Given $\theta \in B_{Z}$, we have

$$
\begin{aligned}
& {\left[\theta,\left(\mathcal{A}_{\pi}, \mathcal{B}_{\pi}\right)\right] \in \mathcal{L}_{h^{2+\alpha}\left(\mathbb{H}^{2}\right)}^{e}\left(h^{1+\alpha}\left(\mathbb{H}^{2}\right), h^{\alpha}\left(\mathbb{H}^{2}\right) \times h^{1+\alpha}\right)} \\
& {\left[\widetilde{\theta}, \mathcal{P}_{\pi}\right] \in \mathcal{L}_{h^{2+\alpha}}^{e}\left(h^{1+\alpha}, h^{\alpha}\left(\mathbb{H}^{2}\right)\right)}
\end{aligned}
$$

and there is a positive constant $C:=C_{Z}$ such that

$$
\left\|\left[\theta,\left(\mathcal{A}_{\pi}, \mathcal{B}_{\pi}\right)\right]^{e}\right\|_{\mathcal{L}\left(h^{1+\alpha}\left(\mathbb{H}^{2}\right), h^{\alpha}\left(\mathbb{H}^{2}\right) \times h^{1+\alpha}\right)}+\left\|\left[\widetilde{\theta}, \mathcal{P}_{\pi}\right]^{e}\right\|_{\mathcal{L}\left(h^{1+\alpha}, h^{\alpha}\left(\mathbb{H}^{2}\right)\right)} \leq C
$$

for all $\theta \in B_{Z}$.

b) Assume that $\theta \in B_{Z}, g \in K$, and that $\beta \in(0, \alpha)$. Then

$$
[\theta, \mathcal{B T}], \quad[\theta, \mathcal{B S P}], \quad[\theta, \partial \mathcal{B}[\cdot, v]] \in \mathcal{L}_{h^{2+\alpha}}^{e}\left(h^{2+\beta}, h^{1+\alpha}\right)
$$


and there is a positive constant $C:=C(Z, K, \beta)$ such that

$\left\|[\theta, \mathcal{B} \mathcal{T}]^{e}\right\|_{\mathcal{L}\left(h^{2+\beta}, h^{1+\alpha}\right)}+\left\|[\theta, \mathcal{B S P}]^{e}\right\|_{\mathcal{L}\left(h^{2+\beta}, h^{1+\alpha}\right)}+\left\|[\theta, \partial \mathcal{B}[\cdot, v]]^{e}\right\|_{\mathcal{L}\left(h^{2+\beta}, h^{1+\alpha}\right)} \leq C$ for all $\theta \in B_{Z}$ and $g \in K$.

Proof. The assertions in a) follow easily from Leibniz' rule.

b) From the definition of $\mathcal{R}$, see (3.10), it follows that

$$
\left(\mathcal{A}, \gamma_{0},(c+g) \mathcal{B}_{1}\right)[\theta, \mathcal{R}]=\left([\mathcal{A}, \theta], 0,(c+g)\left[\mathcal{B}_{1}, \theta\right]\right) \mathcal{R}
$$

Moreover, using again Leibniz' rule, it is easily seen that

$$
[\mathcal{A}, \theta] \in \mathcal{L}\left(h^{2+\beta}(\Omega), h^{\alpha}(\Omega)\right), \quad(c+g)\left[\mathcal{B}_{1}, \theta\right] \in \mathcal{L}\left(h^{2+\beta}(\Omega), h^{1+\alpha}\right)
$$

and that there is a positive constant $C:=C(Z, K)$ such that

$$
\|[\mathcal{A}, \theta]\|_{\mathcal{L}\left(h^{2+\beta}(\Omega), h^{\alpha}(\Omega)\right)}+\left\|(c+g)\left[\mathcal{B}_{1}, \theta\right]\right\|_{\mathcal{L}\left(h^{2+\beta}(\Omega), h^{1+\alpha}\right)} \leq C
$$

for all $\theta \in B_{Z}$ and $g \in K$. We now infer from (6.11) and Theorem 3.5b) that there is a $C:=C(K, Z)>0$ such that

$$
\begin{aligned}
& \|[\theta, \mathcal{R}](f, h, 0)\|_{2+\alpha, \Omega} \\
& \quad \leq C\left\{\|[\mathcal{A}, \theta] \mathcal{R}(f, h, 0)\|_{\alpha, \Omega}+\left\|(c+g)\left[\mathcal{B}_{1}, \theta\right] \mathcal{R}(f, h, 0)\right\|_{1+\alpha}\right\}
\end{aligned}
$$

for all $\theta \in B_{Z}, g \in K, f \in h^{\alpha}(\Omega)$, and $h \in h^{2+\alpha}$. Combining (6.12), (6.14), and the fact that

$$
\mathcal{R} \in C\left(K, \mathcal{L}\left(h^{\beta}(\Omega) \times h^{2+\beta} \times h^{1+\beta}, h^{2+\beta}(\Omega)\right)\right), \quad \beta \in(0, \alpha),
$$

see Theorem 3.5, we find a positive constant $C:=C(Z, K, \beta)$ such that

$$
\|[\theta, \mathcal{R}](f, h, 0)\|_{2+\alpha, \Omega} \leq C\left\{\|f\|_{\beta, \Omega}+\|h\|_{2+\beta}\right\}
$$

for all $\theta \in B_{Z}, g \in K, \beta \in(0, \alpha), f \in h^{\alpha}(\Omega)$, and $h \in h^{2+\alpha}$. In particular, (6.15) says that

$$
[\theta, \mathcal{S}] \in \mathcal{L}_{h^{\alpha}(\Omega)}^{e}\left(h^{\beta}(\Omega), h^{2+\alpha}(\Omega)\right), \quad[\theta, \mathcal{T}] \in \mathcal{L}_{h^{2+\alpha}}^{e}\left(h^{2+\beta}, h^{2+\alpha}(\Omega)\right)
$$

and that there is a constant $C>0$ such that

$$
\left\|[\theta, \mathcal{S}]^{e}\right\|_{\mathcal{L}\left(h^{\beta}(\Omega), h^{2+\alpha}(\Omega)\right)}+\left\|[\theta, \mathcal{T}]^{e}\right\|_{\mathcal{L}\left(h^{2+\beta}, h^{2+\alpha}(\Omega)\right)} \leq C
$$

for all $\theta \in B_{Z}, g \in K$, and $\beta \in(0, \alpha)$. Now the assertions follow from (6.17), the identities

$$
\begin{aligned}
& {[\theta, \mathcal{B} \mathcal{T}]=[\theta, \mathcal{B}] \mathcal{T}+\mathcal{B}[\theta, \mathcal{T}]} \\
& {[\theta, \mathcal{B S P}]=[\widetilde{\theta}, \mathcal{B}] \mathcal{S P}+\mathcal{B}[\widetilde{\theta}, \mathcal{S}] \mathcal{P}+\mathcal{B S}[\widetilde{\theta}, \mathcal{P}]}
\end{aligned}
$$

and again Leibniz' rule. 
Lemma 6.6. Given $\theta \in \mathcal{D}\left(\mathbb{R}^{2}\right)$, we have

$$
\begin{gathered}
\text { a) } \quad \theta \mathcal{T}-\mathcal{T}_{\pi} \theta=\mathcal{S}_{\pi}\left\{\left[\mathcal{A}_{\pi}, \theta\right] \mathcal{T}+\theta\left(\mu_{0}^{2}+\mathcal{A}_{\pi}-\mathcal{A}\right) \mathcal{T}\right\} \\
\text { b) } \quad \theta \mathcal{S P}-\mathcal{S}_{\pi} \mathcal{P}_{\pi} \theta=\mathcal{S}_{\pi}\left\{\left[\mathcal{A}_{\pi}, \theta\right] \mathcal{S P}+\theta\left(\mu_{0}^{2}+\mathcal{A}_{\pi}-\mathcal{A}\right) \mathcal{S P}\right. \\
\left.+\theta\left[\mathcal{P}-\mathcal{P}_{\pi}\right]+\left[\theta, \mathcal{P}_{\pi}\right]\right\} .
\end{gathered}
$$

Proof. Given $h \in h^{2+\alpha}$, define $u_{1}:=\left(\theta \mathcal{T}-\mathcal{T}_{\pi} \theta\right) h$ and $u_{2}:=\left(\theta \mathcal{S P}-\mathcal{S}_{\pi} \mathcal{P}_{\pi} \theta\right) h$. Then $u_{1}, u_{2} \in h^{2+\alpha}\left(\mathbb{H}^{2}\right)$ and (6.9) implies that $\gamma u_{1}=\gamma u_{2}=0$, as well as

$$
\begin{aligned}
\left(\mu_{0}^{2}+\mathcal{A}_{\pi}\right) u_{1} & =\left(\mu_{0}^{2}+\mathcal{A}_{\pi}\right) \theta \mathcal{T} h \\
& =\left[\mathcal{A}_{\pi}, \theta\right] \mathcal{T} h+\theta\left(\mu_{0}^{2}+\mathcal{A}_{\pi}\right) \mathcal{T} h-\theta \mathcal{A} \mathcal{T} h \\
& =\left[\mathcal{A}_{\pi}, \theta\right] \mathcal{T} h+\theta\left(\mu_{0}^{2}+\mathcal{A}_{\pi}-\mathcal{A}\right) \mathcal{T} h
\end{aligned}
$$

and

$$
\begin{aligned}
\left(\mu_{0}^{2}+\mathcal{A}_{\pi}\right) u_{2} & =\left(\mu_{0}^{2}+\mathcal{A}_{\pi}\right) \theta \mathcal{S P} h-\mathcal{P}_{\pi} \theta h \\
& =\left[\mathcal{A}_{\pi}, \theta\right] \mathcal{S P} h+\theta\left(\mu_{0}^{2}+\mathcal{A}_{\pi}-\mathcal{A}\right) \mathcal{S P} h+\theta \mathcal{P} h-\mathcal{P}_{\pi} \theta h \\
& =\left[\mathcal{A}_{\pi}, \theta\right] \mathcal{S P} h+\theta\left(\mu_{0}^{2}+\mathcal{A}_{\pi}-\mathcal{A}\right) \mathcal{S P} h+\theta\left(\mathcal{P}-\mathcal{P}_{\pi}\right) h+\left[\theta, \mathcal{P}_{\pi}\right] h
\end{aligned}
$$

Now the assertions follow from Corollary B.11.

Lemma 6.7. Suppose that $K \subset h_{\mathfrak{A}}^{2+\alpha}$ is compact and that $\rho \in(0,1]$. Then there are positive constants $C:=C(K, Z)$ and $C_{\rho}:=C(\rho, K, Z)$ such that

$$
\begin{aligned}
& \text { a) }\left\|\chi\left(\mu_{0}^{2}+\mathcal{A}_{\pi}-\mathcal{A}\right) u\right\|_{\alpha, \Omega}+\left\|\chi\left(\mathcal{B}-\mathcal{B}_{\pi}\right) u\right\|_{1+\alpha} \\
& \leq C \rho^{1-\alpha}\|u\|_{2+\alpha, \Omega}+C_{\rho}\|u\|_{1+\alpha, \Omega} \\
& \text { b) } \quad\left\|\chi\left(\mathcal{P}-\mathcal{P}_{\pi}\right) h\right\|_{\alpha, \Omega}+\left\|\chi\left(\partial \mathcal{B}[\cdot, v]-A_{\pi, 2}\right) h\right\|_{1+\alpha} \\
& \leq C \rho^{1-\alpha}\|h\|_{2+\alpha}+C_{\rho}\|h\|_{1+\alpha}
\end{aligned}
$$

for all $u \in h^{2+\alpha}(\Omega), h \in h^{2+\alpha}, g \in K$, and $l \in \mathbb{N}$, and where $\left\{\left(U_{l}, \theta_{l}, \chi_{l}\right) ; l \in \mathbb{N}\right\}$ stands for an extended $\rho$-localization sequence for $S$.

Proof. i) From Lemma 2.2 we infer that there is a $C:=C(K)>0$ such that

$$
\left\|a_{j k}(g)\right\|_{1+\alpha, \Omega}+\left\|b_{j, 0}(g)\right\|_{1+\alpha}+\left\|a_{2}(g)\right\|_{\alpha, \Omega} \leq C \quad 1 \leq j, k \leq 2
$$

for all $g \in K$. Since $a_{j k}(g)\left(x_{l}, 0\right)=a_{j k}\left(g, x_{l}\right)$ and $b_{j, 0}(g)\left(x_{l}\right)=b_{j}\left(g, x_{l}\right), 1 \leq j, k \leq$ 2 , see (5.1), the mean value theorem implies that there is a positive constant $C:=C(K)$, independent of $\rho$, such that

$$
\begin{aligned}
& \left\|a_{j k}(g)-a_{j k}\left(g, x_{l}\right)\right\|_{0, \Omega \cap U_{l}}+\left\|b_{j, 0}(g)-b_{j}\left(g, x_{l}\right)\right\|_{0, \mathbb{R} \cap U_{l}} \leq C \rho \\
& {\left[a_{j k}(g)-a_{j k}\left(g, x_{l}\right)\right]_{\alpha, \Omega \cap U_{l}}+\left[b_{j, 0}(g)-b_{j}\left(g, x_{l}\right)\right]_{\alpha, \mathbb{R} \cap U_{i}} \leq C \rho^{1-\alpha}}
\end{aligned}
$$


for all $l \in \mathbb{N}, g \in K$, and $1 \leq j, k \leq 2$. Hence, $(6.7),(6.8),(6.18)$, and (6.19) yield

$$
\begin{aligned}
& \left\|\chi\left(\mu_{0}^{2}+\mathcal{A}_{\pi}-\mathcal{A}\right) u\right\|_{\alpha, \Omega} \\
& \leq \sum_{j, k=1}^{2}\left\|\chi_{l}\left(a_{j k}(g)-a_{j k}\left(g, x_{l}\right)\right) \partial_{j} \partial_{k} u\right\|_{\alpha, \Omega \cap U_{l}} \\
& \quad+Z_{\rho}\left(\mu_{0}\|u\|_{\alpha, \Omega}+\left\|a_{2}(g) \partial_{2} u\right\|_{\alpha, \Omega}\right) \\
& \leq \sum_{j, k=1}^{2}\left\{\left(\left\|\chi_{l}\right\|_{0, \Omega}\left\|a_{j k}(g)-a_{j k}\left(g, x_{l}\right)\right\|_{0, \Omega \cap U_{l}}\right.\right. \\
& \quad+\left\|\chi_{l}\right\|_{0, \Omega}\left[a_{j k}(g)-a_{j k}\left(g, x_{l}\right)\right]_{\alpha, \Omega \cap U_{l}} \\
& \left.\left.\quad+\left[\chi_{l}\right]_{\alpha, \Omega \cap U_{l}}\left\|a_{j k}(g)-a_{j k}\left(g, x_{l}\right)\right\|_{0, \Omega \cap U_{l}}\right)\left\|\partial_{j} \partial_{k} u\right\|_{\alpha, \Omega}\right\} \\
& \quad+Z_{\rho}\left(\mu_{0}\|u\|_{\alpha, \Omega \cap U_{l}}+\left\|a_{2}(g) \partial_{2} u\right\|_{\alpha, \Omega \cap U_{l}}\right) \\
& \leq C\left\{\rho+\rho^{1-\alpha}+\rho^{-\alpha} \rho\right\} \max _{1 \leq j, k \leq 2}\left\|\partial_{j} \partial_{k} u\right\|_{\alpha, \Omega}+C_{\rho}\|u\|_{1+\alpha, \Omega} \\
& \leq C \rho^{1-\alpha}\|u\|_{2+\alpha, \Omega}+C_{\rho}\|u\|_{1+\alpha, \Omega}
\end{aligned}
$$

for all $u \in h^{2+\alpha}(\Omega), g \in K$, and $l \in \mathbb{N}$. This proves the first assertion in a).

ii) Analogously, we have for the boundary operators:

$$
\begin{aligned}
& \left\|\chi\left(\mathcal{B}-\mathcal{B}_{\pi}\right) u\right\|_{1+\alpha} \\
& =\left\|\chi_{l}\left(\mathcal{B}_{0}(g)-\mathcal{B}_{\pi}\left(g, x_{l}\right)\right) u\right\|_{0, \mathbb{R} \cap U_{l}}+\left\|\partial\left[\chi_{l}\left(\mathcal{B}_{0}(g)-\mathcal{B}_{\pi}\left(g, x_{l}\right)\right) u\right]\right\|_{\alpha, \mathbb{R} \cap U_{l}} \\
& \leq\left\|\chi_{l}\left(\mathcal{B}_{0}(g)-\mathcal{B}_{\pi}\left(g, x_{l}\right)\right) u\right\|_{0}+\left\|\partial\left[\chi_{l}\left(\mathcal{B}_{0}(g)-\mathcal{B}_{\pi}\left(g, x_{l}\right)\right) u\right]\right\|_{0, \mathbb{R} \cap U_{l}} \\
& +\left[\partial\left[\chi_{l}\left(\mathcal{B}_{0}(g)-\mathcal{B}_{\pi}\left(g, x_{l}\right)\right) u\right]\right]_{\alpha, \mathbb{R} \cap U_{l}} \\
& \leq \sum_{j=1}^{2}\left\{\left(\left\|\chi_{l}\left(b_{j, 0}(g)-b_{j}\left(g, x_{l}\right)\right)\right\|_{1}\left\|\gamma \partial_{j} u\right\|_{0}\right.\right. \\
& +\left\|\chi_{l}\left(b_{j, 0}(g)-b_{j}\left(g, x_{l}\right)\right)\right\|_{0, \mathbb{R} \cap U_{l}}\left\|\partial \gamma \partial_{j} u\right\|_{0} \\
& +\left[\partial\left[\chi_{l}\left(b_{j, 0}(g)-b_{j}\left(g, x_{l}\right)\right)\right] \gamma \partial_{j} u\right]_{\alpha} \\
& \left.+\left[\chi_{l}\left(b_{j, 0}(g)-b_{j}\left(g, x_{l}\right)\right) \partial \gamma \partial_{j} u\right]_{\alpha, \mathbb{R} \cap U_{l}}\right\} \\
& \leq \sum_{j=1}^{2}\left\{2\left\|\chi_{l}\left(b_{j, 0}(g)-b_{j}\left(g, x_{l}\right)\right)\right\|_{1+\alpha}\left\|\gamma \partial_{j} u\right\|_{\alpha}\right. \\
& +\left\|\chi_{l}\right\|_{0}\left\|b_{j, 0}(g)-b_{j}\left(g, x_{l}\right)\right\|_{0, \mathbb{R} \cap U_{l}}\left\|\partial \gamma \partial_{j} u\right\|_{\alpha} \\
& +\left(\left[\chi_{l}\right]_{\alpha, \mathbb{R} \cap U_{l}}\left\|b_{j, 0}(g)-b_{j}\left(g, x_{l}\right)\right\|_{0, \mathbb{R} \cap U_{l}}\right. \\
& \left.\left.+\left\|\chi_{l}\right\|_{0, \mathbb{R} \cap U_{l}}\left[b_{j, 0}(g)-b_{j}\left(g, x_{l}\right)\right]_{\alpha_{j}, \mathbb{R} \cap U_{l}}\right)\left\|\partial \gamma \partial_{j} u\right\|_{0}\right\} \\
& \leq C\left\{\rho+\rho^{-\alpha} \rho+\rho^{1-\alpha}\right\} \max _{1 \leq j \leq 2}\left\|\partial \gamma \partial_{j} u\right\|_{\alpha}+C_{\rho} \max _{1 \leq j \leq 2}\left\|\gamma \partial_{j} u\right\|_{\alpha} \\
& \leq C \rho^{1-\alpha}\|u\|_{2+\alpha, \Omega}+C_{\rho}\|u\|_{1+\alpha, \Omega} \text {. }
\end{aligned}
$$

This completes the proof of a).

iii) It follows from Lemma 3.4 that

$$
\mathcal{P}(g) h=w(g) \partial^{2} h+N(g) h, \quad g \in K, h \in h^{2+\alpha},
$$


where $w$ is given in (5.6) and where

$$
N(\cdot) \in C\left(K, \mathcal{L}\left(h^{1+\alpha}, h^{\alpha}\right)\right) .
$$

Now, Lemma 5.2b), (6.7), and (6.8) imply

$$
\begin{aligned}
\left\|\chi\left(\mathcal{P}-\mathcal{P}_{\pi}\right) h\right\|_{\alpha, \Omega}= & \| \chi_{l}\left(w(g) \partial^{2} h-\mathcal{P}_{\pi}(g) h+N(g) h \|_{0}\right. \\
& +\left[\chi_{l}\left(w(g) \partial^{2} h-\mathcal{P}_{\pi}(g) h+N(g) h\right]_{\alpha, \Omega \cap U_{l}}\right. \\
\leq & C \rho\|h\|_{2+\alpha}+C_{K}\|h\|_{1+\alpha}+C \rho^{1-\alpha}\|h\|_{2+\alpha}
\end{aligned}
$$

which shows the first part in b).

iv) Let

$$
c_{g}:=-\gamma \partial_{1} v_{g}-\frac{2 \partial g}{c+g} \gamma \partial_{2} v_{g}
$$

cf. (5.5), and observe again that by Lemma 3.4

$$
\partial \mathcal{B}_{0}(g)\left[\cdot, v_{g}\right] h=c_{g} \partial h+M(g) h, \quad h \in h^{2+\alpha}
$$

for some $M \in C\left(K, \mathcal{L}\left(h^{1+\alpha}\right)\right)$. Since $c_{g} \in h^{1+\alpha}$ and since $c_{g}\left(x_{l}\right)=c_{\pi}\left(g, x_{l}\right)$ there is a constant $C:=C(K)>0$ such that

$$
\left\|c_{g}-c_{\pi}\left(g, x_{l}\right)\right\|_{0, U_{l}}+\rho^{\alpha}\left[c_{g}-c_{\pi}\left(g, x_{l}\right)\right]_{\alpha, U_{l}} \leq C \rho
$$

for all $l \in \mathbb{N}$ and all $g \in K$. Now, recall that

$$
A_{\pi, 2}\left(g, x_{l}\right) h=c_{\pi}\left(g, x_{l}\right) \partial h \text { for } h \in h^{2+\alpha} .
$$

Finally, using (6.20), we conclude similarly as in step ii):

$$
\begin{aligned}
\left\|\chi\left(\partial \mathcal{B}[\cdot, v]-A_{\pi, 2}\right) h\right\|_{1+\alpha} \leq & \left\|\chi_{l}\left(c_{g}-c_{\pi}\left(g, x_{l}\right)\right) \partial h+\chi_{l} M(g) h\right\|_{0} \\
& +\left\|\partial\left[\chi_{l}\left(c_{g}-c_{\pi}\left(g, x_{l}\right)\right) \partial h+\chi_{l} M(g) h\right]\right\|_{\alpha, \mathbb{R} \cap U_{l}} \\
\leq & C_{\rho}\|h\|_{1+\alpha}+\left\|\chi_{l}\left(c_{g}-c_{\pi}\left(g, x_{l}\right)\right)\right\|_{\alpha, \mathbb{R} \cap U_{l}}\left\|\partial^{2} h\right\|_{\alpha} \\
\leq & C \rho^{1-\alpha}\|h\|_{2+\alpha}+C_{\rho}\|h\|_{1+\alpha},
\end{aligned}
$$

and the proof of Lemma 6.7 is complete.

After these technical preparations we now give the

Proof of Lemma 6.1. Let $\beta \in(0, \alpha)$ and $\kappa>0$ be given, and suppose that $K \subset h_{\mathfrak{a}}^{2+\alpha}$ is compact. From (6.10) we know that

$$
\theta_{j} \partial \Phi_{t}(g)-A_{\pi, t}\left(g, x_{j}\right) \theta_{j}=\chi_{j}\left[\theta_{j}, \partial \Phi_{t}(g)\right]+\left(\chi_{j} \partial \Phi_{t}(g)-A_{\pi, t}\left(g, x_{j}\right) \chi_{j}\right) \theta_{j} .
$$

Since

$$
\left[\theta_{j}, \partial \Phi_{t}(g)\right]=\left[\theta_{j}, \mathcal{B}_{0}(g) \mathcal{T}(g)+t \partial \mathcal{B}_{0}(g)[\cdot, v]-t \mathcal{B}_{0}(g) \mathcal{S}(g) \mathcal{P}(g)\right],
$$


we infer from Lemma 6.5b) and (6.8) that there is $C_{\rho}:=C(\rho, K, Z, \beta)>0$ such that

$$
\left\|\chi_{j}\left[\theta_{j}, \partial \Phi_{t}(g)\right] h\right\|_{1+\alpha} \leq C_{\rho}\|h\|_{2+\beta}
$$

for all $h \in h^{2+\alpha}, j \in \mathbb{N}, g \in K$, and $t \in[0,1]$. Furthermore, we have

$$
\chi \mathcal{B T}-\mathcal{B}_{\pi} \mathcal{I}_{\pi} \chi=\chi\left(\mathcal{B}-\mathcal{B}_{\pi}\right) \mathcal{T}+\left[\chi, \mathcal{B}_{\pi}\right] \mathcal{T}+\mathcal{B}_{\pi}\left(\chi \mathcal{T}-\mathcal{T}_{\pi} \chi\right)
$$

Hence Lemma 6.6a) gives

$$
\begin{aligned}
\chi \mathcal{B} \mathcal{T}-\mathcal{B}_{\pi} \mathcal{T}_{\pi} \chi= & \chi\left(\mathcal{B}-\mathcal{B}_{\pi}\right) \mathcal{T}+\left[\chi, \mathcal{B}_{\pi}\right] \mathcal{T} \\
& +\mathcal{B}_{\pi} \mathcal{S}_{\pi}\left\{\left[\mathcal{A}_{\pi}, \chi\right] \mathcal{T}+\chi\left(\mu_{0}^{2}+\mathcal{A}_{\pi}-\mathcal{A}\right) \mathcal{T}\right\}
\end{aligned}
$$

Similarly,

$$
\chi \mathcal{B S P}-\mathcal{B}_{\pi} \mathcal{S}_{\pi} \mathcal{P}_{\pi} \chi=\chi\left(\mathcal{B}-\mathcal{B}_{\pi}\right) \mathcal{S P}+\left[\chi, \mathcal{B}_{\pi}\right] \mathcal{S P}+\mathcal{B}_{\pi}\left(\chi \mathcal{S P}-\mathcal{S}_{\pi} \mathcal{P}_{\pi} \chi\right)
$$

and Lemma $6.6 \mathrm{~b}$ ) yields

$$
\begin{aligned}
\chi \mathcal{B S P}-\mathcal{B}_{\pi} \mathcal{S}_{\pi} \mathcal{P}_{\pi} \chi= & \chi\left(\mathcal{B}-\mathcal{B}_{\pi}\right) \mathcal{S P}+\left[\chi, \mathcal{B}_{\pi}\right] \mathcal{S P} \\
& +\mathcal{B}_{\pi} \mathcal{S}_{\pi}\left\{\left[\mathcal{A}_{\pi}, \chi\right] \mathcal{S P}+\chi\left(\mu_{0}^{2}+\mathcal{A}_{\pi}-\mathcal{A}\right) \mathcal{S P}\right. \\
& \left.+\chi\left(\mathcal{P}-\mathcal{P}_{\pi}\right)+\left[\chi, \mathcal{P}_{\pi}\right]\right\} .
\end{aligned}
$$

Finally,

$$
\chi \partial \mathcal{B}[\cdot, v]-A_{\pi, 2} \chi=\chi\left(\partial \mathcal{B}[\cdot, v]-A_{\pi, 2}\right)+\left[\chi, A_{\pi, 2}\right] .
$$

Combining (6.23)-(6.25) (with the parameter $t$ ) one easily gets a representation for $\chi_{j} \partial \Phi_{t}(g)-A_{\pi, 2}\left(g, x_{j}\right) \chi_{j}$. Consequently, it remains to estimate (6.23)-(6.25). We illustrate the procedure for (6.23): From Theorem 3.5b), Remark B.14, Lemma 2.2 , and the fact that $K$ is compact we conclude that there is a constant $C:=$ $C(K, \delta)>0$ such that

$$
\|\mathcal{T}(g)\|_{\mathcal{L}\left(h^{2+\delta}, h^{2+\delta}(\Omega)\right)}+\left\|\mathcal{B}_{\pi}\left(g, x_{j}\right) \mathcal{S}_{\pi}\left(g, x_{j}\right)\right\|_{\mathcal{L}\left(h^{\alpha}\left(\mathbb{E} \mathbb{E}^{2}\right), h^{1+\alpha}\right)} \leq C
$$

for all $g \in K, j \in \mathbb{N}$ and $\delta \in(0,1)$. Consequently, (6.23), (6.26) (applied to $\delta=\alpha$ and $\delta=\beta$ ), Lemma 6.7, and Lemma 6.5 imply

$$
\begin{aligned}
\left\|\left(\chi_{j} \mathcal{B}_{0}(g) \mathcal{T}(g)-\mathcal{B}_{\pi}\left(g, x_{j}\right) \mathcal{T}_{\pi}\left(g, x_{j}\right) \chi_{j}\right) \theta_{j} h\right\|_{1+\alpha} \\
\leq\left\|\chi_{j}\left(\mathcal{B}_{0}(g)-\mathcal{B}_{\pi}\left(g, x_{j}\right)\right) \mathcal{T}(g) \theta_{j} h\right\|_{1+\alpha} \\
\quad+\left\|\left[\chi_{j}, \mathcal{B}_{\pi}\left(g, x_{j}\right)\right] \mathcal{T}(g) \theta_{j} h\right\|_{1+\alpha} \\
\quad+\| \mathcal{B}_{\pi}\left(g, x_{j}\right) \mathcal{S}_{\pi}\left(g, x_{j}\right)\left\{\left[\mathcal{A}_{\pi}\left(g, x_{j}\right), \chi_{j}\right] \mathcal{T}(g)\right. \\
\left.\quad+\chi_{j}\left(\mu_{0}^{2}+\mathcal{A}_{\pi}\left(g, x_{j}\right)-\mathcal{A}(g)\right) \mathcal{T}(g) \theta_{j} h\right\} \|_{1+\alpha} \\
\leq C \rho^{1-\alpha}\left\|\theta_{j} h\right\|_{2+\alpha}+C_{\rho}\|h\|_{2+\beta}
\end{aligned}
$$


for all $h \in h^{2+\alpha}, g \in K, j \in \mathbb{N}$, and $\rho \in(0,1]$. Since $C=C(K)$ is independent of $\rho$, we find $\rho \in(0,1]$ such that

$$
\begin{aligned}
\|\left(\chi_{j} \mathcal{B}_{0}(g) \mathcal{T}(g)-\mathcal{B}_{\pi}\left(g, x_{j}\right)\right. & \left.\mathcal{T}_{\pi}\left(g, x_{j}\right) \chi_{j}\right) \theta_{j} h \|_{1+\alpha} \\
& \leq \frac{\kappa}{2}\left\|\theta_{j} h\right\|_{2+\alpha}+C_{\rho}\|h\|_{2+\beta}
\end{aligned}
$$

for all $h \in h^{2+\alpha}, g \in K$, and $j \in \mathbb{N}$. Similar arguments yield for (6.24) and (6.25):

$$
\begin{gathered}
t\left\{\left\|\left(\chi_{j} \mathcal{B}_{0}(g) \mathcal{S}(g) \mathcal{P}(g)-\mathcal{B}_{\pi}\left(g, x_{j}\right) \mathcal{S}_{\pi}\left(g, x_{j}\right) \mathcal{P}_{\pi}\left(g, x_{j}\right) \chi_{j}\right) \theta_{j} h\right\|_{1+\alpha}\right. \\
+\left\|\chi_{j}\left(\partial \mathcal{B}_{0}(g)\left[\theta_{j} h, v_{g}\right]-A_{\pi, 2}\left(g, x_{j}\right) \theta_{j} h\right)\right\|_{1+\alpha} \\
\left.\times\left\|\left[\chi_{j}, A_{\pi, 2}\left(g, x_{j}\right)\right] \theta_{j} h\right\|_{1+\alpha}\right\} \\
\leq \frac{\kappa}{2}\left\|\theta_{j} h\right\|_{2+\alpha}+C_{\rho}\|h\|_{2+\beta}
\end{gathered}
$$

for all $h \in h^{2+\alpha}, g \in K, t \in[0,1]$, and $j \in \mathbb{N}$. Now the assertion follows from $(6.21),(6.22),(6.27)$, and (6.28).

We are now ready to prove Theorem 1. The main tool here is Corollary 6.3 which enables us to apply the theory of maximal regularity due to Da Prato \& Grisvard $[9]$, see also $[4,20]$, to guarantee the existence of a unique classical solution of problem $(E)_{g_{0}}$. The first proof of the smooth dependence of the semiflow, based on the implicit function theorem, was first given by Angenent in [6], see also [24, 25 ] for some refinements and improvements.

Proof of Theorem 1. a) Let $f_{0} \in V_{c}$ be given and set $g_{0}:=f_{0}-c$. Observe that $g_{0} \in W_{\alpha, 1}=W$. It follows from Lemmas 2.1 and 4.1 that we only have to prove that there exist $t^{+}>0$ and a unique maximal classical solution of $(E)_{g_{0}}$ on $\left[0, t^{+}\right)$ satisfying

$$
\lim _{t \rightarrow t^{+}}\|g(t, \cdot)\|_{2+\alpha}=\infty \quad \text { or } \quad \lim _{t \rightarrow t^{+}} \inf _{h \in \partial W}\|g(t, \cdot)-h\|_{2+\alpha}=0
$$

if $t^{+}<\infty$ and $g \in U C\left(\left[0, t^{+}\right), W\right)$.

b) It follows from Lemma 5.10 that $W$ is an open subset of $h_{\mathfrak{A}}^{2+\alpha}$. Hence, thanks to Lemma 4.3 and Corollary 6.3 , we know that $\Phi \in C^{\infty}\left(W, h^{1+\alpha}\right)$ and that

$$
\partial \Phi(g) \in \mathcal{H}\left(h^{2+\alpha}, h^{1+\alpha}\right), \quad g \in W .
$$

Let now $\beta \in(0, \alpha)$ be fixed and observe that $W=W_{\alpha, 1} \subset W_{\beta, 1}$. Thus the very same arguments as above also ensure that

$$
\partial \Phi(g) \in \mathcal{H}\left(h^{2+\beta}, h^{1+\beta}\right), \quad g \in W .
$$

It is not difficult to see that the maximal $h^{1+\alpha}$-realization of $\partial \Phi(g) \in \mathcal{L}\left(h^{2+\beta}\right.$, $\left.h^{1+\beta}\right), g \in W$, is just the linear operator in (6.30). 
Note that

$$
\left(h^{1+\beta}, h^{2+\beta}\right)_{\alpha-\beta, \infty}^{0}=h^{1+\alpha},
$$

where $(\cdot, \cdot)_{\theta, \infty}^{0}, \theta \in(0,1)$, denotes the continuous interpolation method of Da Prato and Grisvard. Consequently, invoking Theorem 2.3 in [25], we find that

$$
\partial \Phi(g) \in \mathcal{M}_{1}\left(h^{2+\alpha}, h^{1+\alpha}\right), \quad g \in W,
$$

where $\mathcal{M}_{1}\left(X_{1}, X_{0}\right)$ denotes the class of all operators in $\mathcal{L}\left(X_{1}, X_{0}\right)$, having the property of maximal regularity in the sense of Da Prato and Grisvard [9], see also $[6,19,24]$. The existence of a unique maximal classical solution of $(E)_{g_{0}}$ and the property of a smooth semiflow on $W$ can now be obtained along the lines of the proofs of Proposition 3.5 and Theorem 3.2 in [25].

c) Finally suppose that $t^{+}<\infty, g \in U C\left(\left[0, t^{+}\right), W\right)$, and that $(6.29)$ is not true. Then $g_{1}:=\lim _{t \rightarrow t^{+}} g(t)$ exists in $W$. Hence taking $g_{1}$ as initial value in $(E)_{g_{1}}$ one easily constructs a solution $\bar{g}$ of $(E)_{g_{0}}$ extending $g$. This contradicts the maximality of $g$.

Remark 6.8. In the above proof we have used the theory of maximal regularity in the sense of Da Prato \& Grisvard to find a unique solution of $(E)_{g_{0}}$. A different proof of that fact can be based on results of maximal regularity in singular Hölder spaces, see Lunardi [18, 20].

\section{Appendices}

\section{A. Fourier multipliers and a class of elliptic symbols}

In this section we state various multiplier results, particularly the Mihlin-Hörmander theorem, and we introduce a class of Fourier operators which generate (strongly continuous) analytic semigroups on (little) Hölder spaces. Here in Appendix A, we essentially follow the books of Amann [4] and Triebel [28].

Let us first remark that in this section we exclusively deal with spaces of functions and distributions over $\mathbb{R}$. If $\mathbb{R}$ is replaced with $\mathbb{R}^{n}$ we only have to modify the definition of the space $\mathcal{M}$ below and all results remain true.

Assume that $a \in \mathcal{S}^{\prime}$ and that $u \in \mathcal{S}$. Then $\mathcal{F}^{-1} a \in \mathcal{S}^{\prime}$ and therefore $\mathcal{F}^{-1} a * u$ is again a well-defined element of $\mathcal{S}^{\prime}$. We now define a linear operator as follows:

$$
T_{a}: \mathcal{S} \rightarrow \mathcal{S}^{\prime}, \quad T_{a} u:=\mathcal{F}^{-1} a \mathcal{F} u:=\frac{1}{\sqrt{2 \pi}} \mathcal{F}^{-1} a * u
$$

It is well-known that the convolution can be extended to various spaces of distributions. Thus, given a Banach space $E$ with $\mathcal{S} \hookrightarrow E \hookrightarrow \mathcal{S}^{\prime}$, we set

$$
M_{E}:=\left(\left\{a \in \mathcal{S}^{\prime} ; \text { there is a } T_{a}^{e} \in \mathcal{L}(E) \text { such that } T_{a}^{e} \mid \mathcal{S}=T_{a}\right\},\|\cdot\|_{M_{E}}\right),
$$


where $\|a\|_{M_{E}}:=\left\|T_{a}^{e}\right\|_{\mathcal{L}(E)}$ for $a \in M_{E}$. If no confusion seems likely, we write $T_{a}^{e}=T_{a}=\mathcal{F}^{-1} a \mathcal{F}$ for $a \in M_{E}$. We call $M_{E}$ the space of Fourier multipliers for $E$. To illustrate the above concept, assume that $E=L_{2}$. Then it is well-known that $M_{L_{2}}=L_{\infty}$. However, for general Banach spaces $E$ (including $L_{p}, p \neq 2$ ), an explicit description of $M_{E}$ is far from being known. In order to provide at least some subspace of $M_{E}$, let

$$
\mathcal{M}:=\left(\left\{a \in W_{\infty}^{1} ; \sup _{\xi \in \mathbb{R}} \sqrt{1+\xi^{2}}|\partial a(\xi)|<\infty\right\},\|\cdot\|_{\mathcal{M}}\right)
$$

where $\|a\|_{\mathcal{M}}:=\sup _{\xi \in \mathbb{R}}|a(\xi)| \vee \sup _{\xi \in \mathbb{R}} \sqrt{1+\xi^{2}}|\partial a(\xi)|$ for $a \in \mathcal{M}$. It is easily verified that $\mathcal{M}$ is a Banach algebra with respect to pointwise multiplication. Let us now state the following important multiplier theorem:

Theorem A.1. Let $p \in(1, \infty), q \in[1, \infty), s \in \mathbb{R}, k \in \mathbb{Z}$, and $\alpha \in(0,1)$ be given. Then

$$
\mathcal{M} \hookrightarrow M_{L_{p}} \cap M_{B_{q q}^{s}} \cap M_{B U C^{k+\alpha}} \cap M_{h^{k+\alpha}} .
$$

Proof. i) $\mathcal{M} \hookrightarrow M_{L_{p}}, 1<p<\infty$, follows from the Mihlin-Hörmander multiplier theorem, see Theorem 3, Chapter IV, p. 96 in [27].

ii) Recall that $B U C^{k+\alpha}=B_{\infty \infty}^{k+\alpha}$ for $k \in \mathbb{Z}$ and $\alpha \in(0,1)$, see Theorem 3.1 and (3.3). Hence Section 2.6.1 in [28] yields that $\mathcal{M} \hookrightarrow M_{B_{q q}^{s}} \cap M_{B U C^{k+\alpha}}$.

iii) It remains to prove that $\mathcal{M} \hookrightarrow M_{h^{k+\alpha}}$. Suppose that $k \in \mathbb{Z}, \alpha \in(0,1)$, and let $a \in \mathcal{M}$ be given. Due to ii) and the definition of $h^{k+\alpha}$ it suffices to show that $\mathcal{F}^{-1} a \mathcal{F} u \in h^{k+\alpha}$ for $u \in h^{k+\alpha}$. Given $u \in h^{k+\alpha}$, there exists a sequence $\left(u_{n}\right)$ in $\mathcal{S}$ such that $u_{n} \rightarrow u$ in $B U C^{k+\alpha}$ as $n \rightarrow \infty$. We know from [28, p. 131] that $B_{11}^{1+k+\alpha} \hookrightarrow B U C^{k+\alpha}$. Hence $\mathcal{M} \hookrightarrow B_{11}^{1+k+\alpha}$ implies that $\mathcal{F}^{-1} a \mathcal{F} u_{n} \in B_{11}^{1+k+\alpha}$ and that

$$
\left\|\mathcal{F}^{-1} a \mathcal{F}\left(u-u_{n}\right)\right\|_{B U C^{k+\alpha}} \leq c\|a\|_{\mathcal{M}}\left\|\left(u-u_{n}\right)\right\|_{B U C^{k+\alpha}},
$$

i.e., $\mathcal{F}^{-1} a \mathcal{F} u_{n} \rightarrow \mathcal{F}^{-1} a \mathcal{F} u$ in $B U C^{k+\alpha}$ as $n \rightarrow \infty$. This completes the proof, since $\mathcal{S} \stackrel{d}{\hookrightarrow} B_{11}^{1+k+\alpha}$.

Let $E_{0}$ and $E_{1}$ be Banach spaces such that $E_{1} \hookrightarrow E_{0}$. We define

$$
\begin{aligned}
\mathcal{H}\left(E_{1}, E_{0}\right):=\left\{A \in \mathcal{L}\left(E_{1}, E_{0}\right) ; \exists \omega>0, \kappa \geq 1: \omega+A \in \operatorname{Isom}\left(E_{1}, E_{0}\right),\right. \\
\left.\kappa^{-1} \leq \frac{\|(\lambda+A) x\|_{E_{0}}}{|\lambda|\|x\|_{E_{0}}+\|x\|_{E_{1}}} \leq \kappa, \quad x \in E_{1} \backslash\{0\}, \quad \operatorname{Re} \lambda \geq \omega\right\} .
\end{aligned}
$$

Suppose that $A \in \mathcal{H}\left(E_{1}, E_{0}\right)$. Then it is known that $-A$ generates an analytic semigroup $\left\{e^{-t A} ; t \geq 0\right\}$ on $E_{0}$. Observe that $E_{1}$ is not assumed to be dense in $E_{0}$. Thus $\left\{e^{-t A} ; t \geq 0\right\}$ is not strongly continuous at $t=0$, in general. More precisely, the semigroup generated by $-A$ is strongly continuous in $E_{0}$ iff $E_{1}$ is dense in $E_{0}$. In the general case, i.e., if $E_{1}$ is not dense in $E_{0}$ one has that $e^{-t A} x \rightarrow x$ in $E_{0}$ as $t \rightarrow 0$ iff $x$ belongs to the closure of $E_{1}$ in $E_{0}$. 
Given $\alpha_{*}>0$, we introduce the following class of parameter dependent, homogeneous symbols

$$
\begin{aligned}
& \mathcal{E l l S}_{1}^{\infty}\left(\alpha_{*}\right):=\left\{a \in C^{\infty}(\mathbb{R} \times(0, \infty), \mathbb{C}) ; a\right. \text { is positively homogeneous } \\
& \left.\quad \text { of degree } 1 \text { and } \operatorname{Re} a(\eta, \mu) \geq \alpha_{*} \sqrt{\eta^{2}+\mu^{2}} \text { for }(\eta, \mu) \in \mathbb{R} \times(0, \infty)\right\} .
\end{aligned}
$$

Given $a \in \mathcal{E l l S}_{1}^{\infty}\left(\alpha_{*}\right)$ and $\mu>0$, we associate with $a$ the following linear operator

$$
A_{\mu}:=\mathcal{F}^{-1} a(\cdot, \mu) \mathcal{F}
$$

Let $\mu>0$ be given. Since $a \in \mathcal{E} l l \mathcal{S}_{1}^{\infty}\left(\alpha_{*}\right)$ is positively homogeneous of degree 1 it is easily verified that $a(\cdot, \mu) \in \mathcal{O}_{M}$ for each $\mu \geq \mu$, i.e., $a(\cdot, \mu) \in C^{\infty}(\mathbb{R})$ and, given $k \in \mathbb{N}$, there are constants $m_{k} \in \mathbb{N}$ and $c_{k} \geq 0$ such that $\left|\partial^{k} a(\eta, \mu)\right| \leq$ $c_{k}\left(1+|\eta|^{2}\right)^{m_{k}}, \eta \in \mathbb{R}, \mu \geq \underline{\mu}$. It is well-known that $a(\cdot, \mu) \in \mathcal{O}_{M}$ implies that

$$
A_{\mu} \in \mathcal{L}(\mathcal{S}) \cap \mathcal{L}\left(\mathcal{S}^{\prime}\right) \quad \text { for } \quad \mu \geq \underline{\mu} .
$$

However, for our purposes we need some further properties of $A_{\mu}$. Particularly, we are interested in subspaces $E$ of $\mathcal{S}^{\prime}$ such that $A_{\mu}(E) \subset h^{k+\alpha}$. The next theorem gives a precise answer to this question. It states that for each symbol in $\mathcal{E l l S}_{1}^{\infty}\left(\alpha_{*}\right)$ the corresponding Fourier operator belongs to the class $\mathcal{H}\left(h^{k+1+\alpha}, h^{k+\alpha}\right)$. This result is a special case of a general theorem due to $\mathrm{H}$. Amann. For a proof we refer to [4]. It uses Theorem A.1 and parameter dependent norms in Besov spaces.

Theorem A.2. Let $\alpha_{*}>0$ and $\underline{\mu}>0$ be given and suppose that $a \in \mathcal{E} l l \mathcal{S}_{1}^{\infty}\left(\alpha_{*}\right)$. Then

$$
\begin{aligned}
& A_{\mu} \in \mathcal{H}\left(B U C^{k+1+\alpha}, B U C^{k+\alpha}\right) \cap \mathcal{H}\left(h^{k+1+\alpha}, h^{k+\alpha}\right), \\
& e^{-a(\cdot, \mu) t} \in \mathcal{M}, \quad \text { and } \quad e^{-t A_{\mu}}=\mathcal{F}^{-1} e^{-a(\cdot, \mu) t} \mathcal{F}
\end{aligned}
$$

for each $\mu \geq \underline{\mu}, k \in \mathbb{Z}, \alpha \in(0,1)$, and $t \geq 0$.

\section{B. Second order elliptic boundary value problems in a half space}

Let $\mathbb{H}^{2}:=\left\{(x, y) \in \mathbb{R}^{2} ; y>0\right\}$ be a half space in $\mathbb{R}^{2}$. Of concern are second order elliptic boundary value problems with constant coefficients in the little Hölder spaces $h^{\alpha}\left(\mathbb{H}^{2}\right)$. In particular, we derive a representation of the solution using socalled Poisson type and singular Green operators.

Let $a_{12}, a_{22} \in \mathbb{R}$ be given and set

$$
p(\xi):=\eta^{2}+2 a_{12} \eta \theta+a_{22} \theta^{2} \quad \text { for } \quad \xi=(\eta, \theta) \in \mathbb{R}^{2},
$$

and

$$
\mathcal{A}_{\pi} u:=-\partial_{1}^{2} u-2 a_{12} \partial_{1} \partial_{2} u-a_{22} \partial_{2}^{2} u \quad \text { for } \quad u \in B U C^{2}\left(\mathbb{H}^{2}\right) \text {. }
$$


Throughout this section let $\mu>0$ be fixed. Moreover, we often use the notation $\mathcal{A}_{\pi, \mu}:=\mu^{2}+\mathcal{A}_{\pi}$.

Given $f \in h^{\alpha}\left(\mathbb{H}^{2}\right)$ and $g \in h^{2+\alpha}$, we are interested in solutions of the following problem

$$
\left(\mu^{2}+\mathcal{A}_{\pi}\right) u=f \quad \text { in } \quad \mathbb{H}^{2}, \quad u(\cdot, 0)=g \quad \text { on } \quad \mathbb{R} .
$$

In order to obtain satisfactory existence results, let us assume that the following ellipticity condition holds: There exists an $\alpha_{0}>0$ with

$$
p(\xi) \geq \alpha_{0}|\xi|^{2} \quad \text { for } \quad \xi \in \mathbb{R}^{2} .
$$

Moreover, we define

$$
q_{\eta}(\mu, z):=\mu^{2}+\eta^{2}+2 i a_{12} \eta z-a_{22} z^{2} \quad \text { for } \eta \in \mathbb{R}, z \in \mathbb{C} .
$$

Remarks B.1. a) We have

$$
a_{22}-a_{12}^{2} \geq \alpha_{0}
$$

Indeed, let $\eta:=-a_{12}, \theta:=1$, and $\xi:=(\eta, \theta)$. Then $(B .3)$ implies that

$$
p(\xi)=a_{12}^{2}-2 a_{12}^{2}+a_{22}=a_{22}-a_{12}^{2} \geq \alpha_{0}\left(1+a_{12}^{2}\right) \geq \alpha_{0} .
$$

b) Given $\eta \in \mathbb{R}$, there is exactly one root of $q_{\eta}(\mu, \cdot)$ with positive real part. It is given by

$$
\lambda(\eta, \mu)=i a(\eta)+d(\eta, \mu)
$$

where

$$
a(\eta):=-\frac{a_{12}}{a_{22}} \eta \quad \text { and } \quad d(\eta, \mu):=\frac{1}{a_{22}}\left[a_{22} \mu^{2}+\left(a_{22}-a_{12}^{2}\right) \eta^{2}\right]^{1 / 2} .
$$

This follows easily from Remark a).

c) Let $\alpha_{*}:=\frac{\sqrt{\alpha_{0}}}{a_{22}}$. Then

$$
\lambda \in \mathcal{E} l l \mathcal{S}_{1}^{\infty}\left(\alpha_{*}\right) .
$$

Indeed, it is obvious that $\lambda \in C^{\infty}(\mathbb{R} \times(0, \infty), \mathbb{C})$ is positively homogeneous of degree 1. Moreover, from Remark a) we know that $a_{22} \geq a_{22}-a_{12}^{2} \geq \alpha_{0}$. This implies that

$$
\begin{aligned}
\operatorname{Re\lambda }(\eta, \mu)=d(\eta, \mu) & =\frac{1}{a_{22}}\left[a_{22} \mu^{2}+\left(a_{22}-a_{12}^{2}\right) \eta^{2}\right]^{1 / 2} \\
& \geq \frac{1}{a_{22}}\left[\alpha_{0} \mu^{2}+\alpha_{0} \eta^{2}\right]^{1 / 2} \geq \frac{\sqrt{\alpha_{0}}}{a_{22}}\left[\mu^{2}+\eta^{2}\right]^{1 / 2}
\end{aligned}
$$

B.1 Representation of the Poisson operator. Given $g \in h^{2+\alpha}$, define

$$
\left(\mathcal{T}_{\pi, \mu} g\right)(x, y):=\left[\mathcal{F}^{-1} e^{-\lambda(\cdot, \mu) y} \mathcal{F} g\right](x) \quad \text { for } \quad(x, y) \in \mathbb{H}^{2}
$$

Operators of the form (B.5) are called Poisson operators, cf. [15]. In the main result of this section, Lemma B.2 below, we show that Poisson operators are in fact solution operators for Dirichlet problems in a half space. 
Lemma B.2. Suppose that $g \in h^{2+\alpha}$. Then the unique solution of

$$
\left(\mu^{2}+\mathcal{A}_{\pi}\right) u=0 \quad \text { in } \mathbb{H}^{2}, \quad u(\cdot, 0)=g \quad \text { on } \quad \mathbb{R}
$$

in the class $h^{2+\alpha}\left(\mathbb{H}^{2}\right)$ is represented by $u:=\mathcal{T}_{\pi, \mu} g$. Moreover,

$$
\mathcal{T}_{\pi, \mu} \in \mathcal{L}\left(h^{2+\alpha}, h^{2+\alpha}\left(\mathbb{H}^{2}\right)\right)
$$

For the proof we need some preparation. Let us start with the following definition.

$$
M_{\mu}(y):=\left\|e^{-\lambda(\cdot, \mu) y}\right\|_{\mathcal{M}} \text { for } \quad y \geq 0
$$

\section{Lemma B.3.}

$$
M_{\mu}(y) \rightarrow 0 \quad \text { as } \quad y \rightarrow \infty \quad \text { and } \quad M_{\mu}:=\sup _{y \geq 0} M_{\mu}(y)<\infty .
$$

Proof. Observe that $\operatorname{Re} \lambda(\eta, \mu) \geq \alpha_{*}\left(\eta^{2}+\mu^{2}\right)^{1 / 2}$ for $\eta \in \mathbb{R}, y \geq 0$, cf. Remark B.1c). Consequently,

$$
\left|e^{-\lambda(\eta, \mu) y}\right| \leq e^{-\alpha_{*} \mu y}
$$

Moreover, since $\partial_{1} \lambda(\cdot, \cdot)$ is homogeneous of degree 0 we find that

$$
\sqrt{1+\eta^{2}}\left|\partial_{1} e^{-\lambda(\eta, \mu) y}\right| \leq c_{\mu} \sqrt{\mu^{2}+\eta^{2}} y e^{-\alpha * y \sqrt{\mu^{2}+\eta^{2}}}
$$

for an appropriate positive constant $c_{\mu}$. This proves the Lemma.

Lemma B.4. Let $\alpha \in(0,1)$ be given. Then

a) $\bigcap_{l=0}^{2}\left\{B U C^{l+\alpha}\left(\dot{\mathbb{R}}_{+}, B U C^{2-l}\right) \cap B^{l}\left(\dot{\mathbb{R}}_{+}, B U C^{2+\alpha-l}\right)\right\}=B U C^{2+\alpha}\left(\mathbb{H}^{2}\right)$

b) $\quad h^{3}\left(\dot{\mathbb{R}}_{+}, h^{3}\right) \hookrightarrow h^{2+\alpha}\left(\mathbb{H}^{2}\right)$

with respect to the identification $[y \mapsto u(\cdot, y)] \mapsto u(\cdot, \cdot)$.

Proof. a) can be found in [4].

b) is implied by

$$
h^{3}\left(\dot{\mathbb{R}}_{+}, h^{3}\right) \hookrightarrow h^{3}\left(\mathbb{H}^{2}\right) \hookrightarrow h^{2+\alpha}\left(\mathbb{H}^{2}\right) .
$$

Proof of Lemma B.2. Let $g \in h^{2+\alpha}$ be given and put

$$
u(x, y):=\left[\mathcal{F}^{-1} e^{-\lambda(\cdot, \mu) y} \mathcal{F} g\right](x) \text { for }(x, y) \in \mathbb{H}^{2} .
$$

a) We first prove that $u \in B U C^{2+\alpha}\left(\mathbb{H}^{2}\right)$.

i) Let $A_{\mu}:=\mathcal{F}^{-1} \lambda(\cdot, \mu) \mathcal{F}$. Then Theorem A.2 and Remark B.1c) imply that

$$
\begin{aligned}
& A_{\mu} \in \mathcal{H}\left(B U C^{k+1+\alpha}, B U C^{k+\alpha}\right), k \in \mathbb{Z} \\
& e^{-\lambda(\cdot, \mu) y} \in \mathcal{M} \text { and } e^{-y A_{\mu}}=\mathcal{F}^{-1} e^{-\lambda(\cdot, \mu) y} \mathcal{F} .
\end{aligned}
$$


From $(B .7)_{k}$ it follows that

$$
\begin{aligned}
& {[y \mapsto u(\cdot, y)] \in C^{k}\left(\dot{\mathbb{R}}_{+}, B U C^{2+\alpha-k}\right)} \\
& \text { with } \partial_{y}^{k} u(\cdot, y)=\left(-A_{\mu}\right)^{k} u(\cdot, y), k \in \mathbb{N}, y>0 .
\end{aligned}
$$

Hence, $(B .7)_{k},(B .8),(B .9)_{k}$, Lemma B.3, and Theorem A.2 guarantee the existence of a positive constant $c:=c(\mu, k)$, independent of $g$, such that

$$
\begin{array}{r}
\left\|\partial_{y}^{k} u(\cdot, y)\right\|_{B U C^{2+\alpha-k}} \leq c\|u(\cdot, y)\|_{B U C^{2+\alpha}}=c\left\|e^{-y A_{\mu}} g\right\|_{B U C^{2+\alpha}} \\
=c\left\|\mathcal{F}^{-1} e^{-\lambda(\cdot, \mu) y} \mathcal{F} g\right\|_{B U C^{2+\alpha}} \leq c M_{\mu}(y)\|g\|_{B U C^{2+\alpha}}
\end{array}
$$

for $k \in \mathbb{N}$ and $y>0$. In particular, we have

$$
[y \mapsto u(\cdot, y)] \in B C^{k}\left(\dot{\mathbb{R}}_{+}, B U C^{2+\alpha-k}\right), \quad k \in \mathbb{N} .
$$

ii) Let $(\cdot, \cdot)_{\theta, p}, \theta \in(0,1), 1 \leq p \leq \infty$, denote the real interpolation functor. Then it follows from Theorem 2.4.2 and Remark 2.7.1.2 in [28] that

$$
\left(B U C^{1+\alpha-k}, B U C^{2+\alpha-k}\right)_{1-\alpha, 1}=B_{\infty 1}^{2-k} \hookrightarrow B U C^{2-k}, \quad k \in\{0,1,2\} .
$$

Thus we infer from $(B .10)_{l},(B .11)_{l},(B .12)_{l}$, the mean value theorem, and by interpolation that

$$
[y \mapsto u(\cdot, y)] \in B U C^{l+\alpha}\left(\dot{\mathbb{R}}_{+}, B U C^{2-l}\right), \quad l \in\{0,1,2\},
$$

and that

$$
\|[y \mapsto u(\cdot, y)]\|_{B U C^{l+\alpha}\left(\dot{\mathbb{R}}_{+}, B U C^{2-l}\right)} \leq c M_{\mu}\|g\|_{2+\alpha}, \quad l \in\{0,1,2\} .
$$

Now Lemma B.4, $(B .14)_{l}$, and $(B .11)_{l}$ for $l \in\{0,1,2\}$ imply that $u$ belongs to $B U C^{2+\alpha}\left(\mathbb{H}^{2}\right)$. This proves a).

In addition, Lemma B.4, $(B .10)_{l}$ and $(B .15)_{l}$ yield the a priori estimate

$$
\|u\|_{B U C^{2+\alpha}\left(\mathbb{H}^{2}\right)}=\left\|\mathcal{T}_{\pi, \mu} g\right\|_{B U C^{2+\alpha}\left(\mathbb{H}^{2}\right)} \leq c M_{\mu}\|g\|_{2+\alpha} .
$$

Since $g \in h^{2+\alpha}$, there is a sequence $\left(g_{n}\right) \subset \mathcal{S}$ such that

$$
g_{n} \rightarrow g \text { in } B U C^{2+\alpha} \text { as } n \rightarrow \infty .
$$

b) Let $u_{n}(x, y):=\left[\mathcal{F}^{-1} e^{-\lambda(\cdot, \mu) y} \mathcal{F} g_{n}\right](x)$ for $(x, y) \in \mathbb{H}^{2}, n \in \mathbb{N}$. Then we claim that

$$
u_{n} \in h^{2+\beta}\left(\mathbb{H}^{2}\right) \text { for any } \beta \in(0,1) .
$$

Recall that for fixed $k \in \mathbb{N}$ and $\alpha \in(0,1)$ we have $\mathcal{M} \hookrightarrow M_{h^{k+\alpha}}$, cf. Theorem A.1. Thus we find that

$$
\begin{aligned}
& {\left[y \mapsto u_{n}(\cdot, y)\right] \in C^{l}\left(\dot{\mathbb{R}}_{+}, h^{k+\alpha}\right)} \\
& \text { with } \partial_{y}^{l} u_{n}(\cdot, y)=\left(-A_{\mu}\right)^{l} u_{n}(\cdot, y), k, l, n \in \mathbb{N}, y>0 .
\end{aligned}
$$


From this and the fact that $A_{\mu} \in \mathcal{H}\left(h^{k+1+\alpha}, h^{k+\alpha}\right), k \in \mathbb{N}$, cf. Theorem A.2, we conclude similarly as in $(B .10)_{k}$ that there is a positive constant $c:=c(k, \mu)$ such that

$$
\left\|\partial_{y}^{k} u_{n}(\cdot, y)\right\|_{h^{3+\alpha}} \leq c M_{\mu}(y)\left\|g_{n}\right\|_{h^{3+k+\alpha}} \quad \text { for } \quad k, n \in \mathbb{N}, y>0 . \quad(B .20)_{k}
$$

Hence Lemma B.3 and $h^{3+\alpha} \hookrightarrow h^{3}$ yield that

$$
\left[y \mapsto u_{n}(\cdot, y)\right] \in h^{3}\left(\dot{\mathbb{R}}_{+}, h^{3}\right)
$$

Now assertion b), i.e., (B.18), follows from Lemma B.4.

c) We assert that

$$
u_{n} \rightarrow u \quad \text { in } B U C^{2+\alpha}\left(\mathbb{H}^{2}\right) \text { as } n \rightarrow \infty
$$

Indeed, this follows immediately from $(B .16),(B .17)$, and $(B .18)$.

Now let $\beta \in(\alpha, 1)$ be fixed. Then we know from Lemma 3.2a) that $h^{2+\alpha}\left(\mathbb{H}^{2}\right)$ is the closure of $h^{2+\beta}\left(\mathbb{H}^{2}\right)$ in $B U C^{2+\alpha}\left(\mathbb{H}^{2}\right)$. Hence $(B .18)$ and $(B .20)$ yield that $u \in h^{2+\alpha}\left(\mathbb{H}^{2}\right)$. This shows, together with $(B .16)$, that

$$
\mathcal{T}_{\pi, \mu} \in \mathcal{L}\left(h^{2+\alpha}, h^{2+\alpha}\left(\mathbb{H}^{2}\right)\right) .
$$

d) In a last step we verify that $u$ is in fact the unique solution of (B.6). Indeed, observe that

$$
\gamma u=u(\cdot, 0)=\left.e^{-y A_{\mu}} g\right|_{y=0}=g,
$$

i.e., $u$ satisfies the boundary condition in (B.6). Furthermore we have

$$
\begin{aligned}
\left(\mu^{2}+\right. & \left.\mathcal{A}_{\pi}\right) u(x, y)=\left(\mu^{2}-\partial_{1}^{2}-2 a_{12} \partial_{1} \partial_{2}-a_{22} \partial_{2}^{2}\right) u(x, y) \\
& =\mathcal{F}^{-1}\left[\eta \mapsto\left\{\mu^{2}+\eta^{2}+2 i a_{12} \eta \lambda(\eta, \mu)-a_{22} \lambda^{2}(\eta, \mu)\right\} e^{-\lambda(\eta, \mu) y}\right] \mathcal{F} g(x) \\
& =\mathcal{F}^{-1} q_{\eta}(\cdot, \lambda(\cdot, \mu)) e^{-\lambda(\cdot, \mu) y} \mathcal{F}_{g}(x)
\end{aligned}
$$

for $(x, y) \in \mathbb{H}^{2}$. But $q_{\mu}(\eta, \lambda(\eta, \mu))=0$ for all $(\eta, \mu) \in \mathbb{R} \times(0, \infty)$. Thus $\left(\mu^{2}+\mathcal{A}_{\pi}\right) u=$ 0 . Therefore, $u$ is a solution of (B.6). Finally, we infer from the Phragmèn-Lindelöf principle, see [23, Theorem 19, Chapter 2], that $u$ is the only solution of $(B .6)$ in $h^{2+\alpha}\left(\mathbb{K}^{2}\right)$. This completes the proof of Lemma B.2.

Remarks B.5. a) Let us rewrite estimate (B.16) in the form

$$
\left\|\mathcal{T}_{\pi, \mu}\right\|_{\mathcal{C}\left(h^{2+\alpha}, h^{2+\alpha}\left(\mathbb{H}^{2}\right)\right)} \leq c M_{\mu}
$$

which can be seen as an a priori estimate for (B.6).

b) Suppose that $g \in \mathcal{S}$. Then the proof of step b) shows that the solution $u=\mathcal{T}_{\pi, \mu} g$ of $(B .6)$ is in $h^{\infty}\left(\mathbb{H}^{2}\right)$. 
c) Assume that $u \in \mathcal{S}\left(\mathbb{H}^{2}\right)$ is a solution of (B.6). Using partial Fourier transform and Lemma B.4a) it follows that $u$ is in fact represented by (B.5). In particular, this gives another proof of the uniqueness assertion in Lemma B.2.

B.2 Representation of the singular Green operator. Suppose that $\alpha \in(0,1)$. Given $u \in B U C^{\alpha}\left(\mathbb{H}^{2}\right)$, let $\mathcal{E}$ denote the following extension operator:

$$
\mathcal{E} u(x, y):=\left\{\begin{array}{lll}
u(x, y) & \text { if } & (x, y) \in \overline{\mathbb{H}}{ }^{2} \\
u(x,-y) & \text { if } & (x,-y) \in \mathbb{H}^{2} .
\end{array}\right.
$$

Observe that $\mathcal{E} \in \mathcal{L}\left(B U C^{\alpha}\left(\mathbb{H}^{2}\right), B U C^{\alpha}\left(\mathbb{R}^{2}\right)\right)$. Moreover, using the fact that $h^{\beta}\left(\mathbb{H}^{2}\right)$ is dense in $h^{\alpha}\left(\mathbb{I}^{2}\right), \beta \in(\alpha, 1)$, it is easily verified that

$$
\mathcal{E} \in \mathcal{L}\left(h^{\alpha}\left(\mathbb{H}^{2}\right), h^{\alpha}\left(\mathbb{R}^{2}\right)\right) .
$$

We introduce some further notation. Let $\mathcal{F}_{2}$ denote the Fourier transform in $\mathcal{S}^{\prime}\left(\mathbb{R}^{2}\right)$. Furthermore, set $\mathcal{S}\left(\mathbb{H}^{2}\right):=r_{\mathbb{H}^{2}}\left(\mathcal{S}\left(\mathbb{R}^{2}\right)\right)$, cf. (3.5). Then, for $f \in \mathcal{S}\left(\mathbb{H}^{2}\right)$, we define

$$
\mathcal{F} f(\eta, y):=\frac{1}{\sqrt{2 \pi}} \int_{\mathbb{R}} e^{-i x \eta} f(x, y) d x, \quad(\eta, y) \in \mathbb{H}^{2} .
$$

Finally, given $f \in \mathcal{S}\left(\mathbb{H}^{2}\right)$ and $\eta \in \mathbb{R}$, put

$$
k_{f, \mu}(\eta):=-\frac{1}{a_{22}} \int_{0}^{\infty} e^{-d(\eta, \mu) y} \cos (a(\eta) y) \mathcal{F} f(\eta, y) d y .
$$

Remarks B.6. a) Suppose that $f \in \mathcal{S}\left(\mathbb{H}^{2}\right)$ and that $y>0$. Then it is easily verified that

$$
\mathcal{F} f(\cdot, y) \in \mathcal{S} \text {, uniformly in } y \text {. }
$$

b) Recall that from Remark B.1c) we know that

$$
\operatorname{Re} \lambda(\eta, \mu) y=d(\eta, \mu) y \geq \alpha_{*} \sqrt{\mu^{2}+\eta^{2}} y \quad \text { for } \quad(\eta, y) \in \mathbb{R} \times(0, \infty) .
$$

Consequently,

$$
k_{f, \mu} \in \mathcal{S} \quad \text { for } \quad f \in \mathcal{S}\left(\mathbb{H}^{2}\right) .
$$

Suppose that $f \in \mathcal{S}\left(\mathbb{H}^{2}\right)$. We define the following operators:

$$
\begin{aligned}
& \mathcal{S}_{\pi, \mu, 1} f:=r_{\mathbb{H} 2} \mathcal{F}_{2}\left(\mu^{2}+p\right)^{-1} \mathcal{F}_{2} \mathcal{E} f \\
& \mathcal{S}_{\pi, \mu, 2} f(x, y):=\left[\mathcal{F}^{-1} \frac{e^{-\lambda(\cdot, \mu) y}}{d(\cdot, \mu)} k_{f, \mu}(\cdot)\right](x), \quad(x, y) \in \mathbb{H}^{2} .
\end{aligned}
$$

Remarks B.7. a) Of course, $\mu^{2}+p$ is the symbol of the elliptic operator $\mathcal{A}_{\pi, \mu}=$ $\mu^{2}+\mathcal{A}_{\pi}$, cf. (B.1), acting on $\mathcal{S}\left(\mathbb{R}^{2}\right)$. Moreover, using Theorem A.1 it is not difficult to see that

$$
\mathcal{A}_{\pi, \mu} \in \operatorname{Isom}\left(h^{2+\alpha}\left(\mathbb{R}^{2}\right), h^{\alpha}\left(\mathbb{R}^{2}\right)\right)
$$


and that

$$
\mathcal{A}_{\pi, \mu}^{-1}=\mathcal{F}_{2}^{-1}\left(\mu^{2}+p\right)^{-1} \mathcal{F}_{2}
$$

Hence, $\mathcal{S}_{\pi, \mu, 1}$ is the restriction of $\mathcal{A}_{\pi, \mu}^{-1}$ to $\mathcal{S}\left(\mathbb{H}^{2}\right)$ in the sense of [15], Definition 1.2 .5$, p. 22 .

b) Assume that $f \in \mathcal{S}\left(\mathbb{H}^{2}\right)$. Then $(B .25)$ and $(B .26)$ imply that $e^{-\lambda(\cdot, \mu) y} / d(\cdot, \mu)$ belongs to $\mathcal{O}_{M}$ for $y \geq 0$ and that $k_{f, \mu} \in \mathcal{S}$. Therefore, $\mathcal{S}_{\pi, \mu, 2} f$ is well-defined and it is not difficult to verify that $\mathcal{S}_{\pi, \mu, 2} f \in C^{\infty}\left(\mathbb{H}^{2}\right)$.

c) Operators of type $\mathcal{S}_{\pi, \mu, 2}$ are called singular Green operators, cf. [15].

Let us now state our main result of this section.

Lemma B.8. $\mathcal{S}_{\pi, \mu, 2}$ possesses a unique extension, denoted again by $\mathcal{S}_{\pi, \mu, 2}$, such that

$$
\mathcal{S}_{\pi, \mu, 2} \in \mathcal{L}\left(h^{\alpha}\left(\mathbb{H}^{2}\right), h^{2+\alpha}\left(\mathbb{H}^{2}\right)\right) .
$$

Moreover, the following representation holds:

$$
\mathcal{S}_{\pi, \mu, 2}=-\mathcal{T}_{\pi, \mu} \gamma \mathcal{S}_{\pi, \mu, 1}
$$

In order to prove the above result, we provide some preparing material. Let us start with the following Lemma:

\section{Lemma B.9.}

$$
\mathcal{S}_{\pi, \mu, 1} \in \mathcal{L}\left(h^{\alpha}\left(\mathbb{H}^{2}\right), h^{2+\alpha}\left(\mathbb{H}^{2}\right)\right) \quad \text { and } \quad \mathcal{A}_{\pi, \mu} \mathcal{S}_{\pi, \mu, 1}=i d_{h^{\alpha}\left(\mathbb{H}^{2}\right)}
$$

Proof. Recall that $\mathcal{E} \in \mathcal{L}\left(h^{\alpha}\left(\mathbb{H}^{2}\right), h^{\alpha}\left(\mathbb{R}^{2}\right)\right)$, cf. $(B .23)$ and that $r_{\mathbb{H}^{2}} \in \mathcal{L}\left(h^{2+\alpha}\left(\mathbb{R}^{2}\right)\right.$, $\left.h^{2+\alpha}\left(\mathbb{H}^{2}\right)\right)$. Hence the first assertion follows from Remark B.7a). Moreover, due to the identity $\partial^{\beta} r_{\mathbb{H}^{2}}=r_{\mathbb{H}^{2}} \partial^{\beta}, \beta \in \mathbb{N}^{n}$, and (B.28), we find that

$$
\mathcal{A}_{\pi, \mu} \mathcal{S}_{\pi, \mu, 1}=\mathcal{A}_{\pi, \mu} r_{\mathbb{H}^{2}} \mathcal{A}_{\pi, \mu}^{-1} \mathcal{E}=r_{\mathbb{H}^{2}} \mathcal{E}=\operatorname{id}_{h^{\alpha}\left(\mathbb{H}^{2}\right)} \text {. }
$$

Lemma B.10. Let $y>0$ and $\eta \in \mathbb{R}$ be given. Then

$$
\int_{\mathbb{R}}\left(\mu^{2}+p(\eta, \theta)\right)^{-1} \cos (y \theta) d \theta=\frac{\pi}{a_{22} d(\eta, \mu)} e^{-d(\eta, \mu) y} \cos (a(\eta) y)
$$

Proof. Fix $y>0$ and $\eta \in \mathbb{R}$. Moreover, let $\psi:=\theta+a(\eta), \theta \in \mathbb{R}$. Observing Remark B.1a), it is not difficult to verify that

$$
\mu^{2}+p(\eta, \theta)=a_{22}\left(d^{2}(\eta, \mu)+\psi^{2}\right) .
$$

Recall that the Fourier transform of the onedimensional Poisson kernel is given by

$$
\int_{\mathbb{R}} \frac{t}{t^{2}+\tau^{2}} e^{i y \tau} d \tau=\pi e^{-|y| t}, \quad t>0, y \in \mathbb{R}
$$


see, for example, [22, p. 71]. Now, substituting $\tau=\theta+a(\eta)$ in the integral in $(B .32)$, it follows from $(B .31)$ that

$$
\int_{\mathbb{R}}\left(\mu^{2}+p(\eta, \theta)\right)^{-1} e^{i y \theta} d \theta=\frac{\pi}{a_{22} d(\eta, \mu)} e^{-\lambda(\eta, \mu) y} .
$$

This formula and its conjugated analogue imply the assertion.

Proof of Lemma B.8. a) From Lemma B.9 we know that $\mathcal{S}_{\pi, \mu, 1} \in \mathcal{L}\left(h^{\alpha}\left(\mathbb{H}^{2}\right)\right.$, $\left.h^{2+\alpha}\left(\mathbb{H}^{2}\right)\right)$ and Lemma. B.2 gives that $\mathcal{T}_{\pi, \mu} \in \mathcal{L}\left(h^{2+\alpha}, h^{2+\alpha}\left(\mathbb{H}^{2}\right)\right)$. Moreover, note that $\gamma \in \mathcal{L}\left(h^{2+\alpha}\left(\mathbb{H}^{2}\right), h^{2+\alpha}\right)$ and that $\mathcal{S}\left(\mathbb{H}^{2}\right) \stackrel{d}{\hookrightarrow} h^{\alpha}\left(\mathbb{H}^{2}\right)$, see $(3.5)$. Hence it suffices to prove that

$$
\mathcal{S}_{\pi, \mu, 2} f=-\mathcal{T}_{\pi, \mu} \gamma \mathcal{S}_{\pi, \mu, 1} f \quad \text { for } \quad f \in \mathcal{S}\left(\mathbb{H}^{2}\right) .
$$

b) In the following let $f \in \mathcal{S}\left(\mathbb{H}^{2}\right)$ be fixed. Then

$$
\gamma \mathcal{S}_{\pi, \mu, 1} f(x)=\frac{1}{2 \pi} \int_{\mathbb{R}^{2}} e^{i x \eta}\left(\mu^{2}+p(\eta, \theta)\right)^{-1} \mathcal{F}_{2} \mathcal{E} f(\eta, \theta) d \eta d \theta, \quad x \in \mathbb{R}
$$

and consequently,

$$
\gamma \mathcal{S}_{\pi, \mu, 1} f(x)=\frac{1}{\sqrt{2 \pi}} \mathcal{F}^{-1}\left[\int_{\mathbb{R}}\left(\mu^{2}+p(\cdot, \theta)\right)^{-1} \mathcal{F}_{2} \mathcal{E} f(\cdot, \theta) d \theta\right](x), \quad x \in \mathbb{R} .
$$

But we also have

$$
\begin{aligned}
\mathcal{F}_{2} \mathcal{E} f(\eta, \theta) & =\frac{1}{2 \pi} \int_{\mathbb{R}^{2}} e^{-i x \eta-i y \theta} \mathcal{E} f(x, y) d x d y \\
& =\frac{1}{\sqrt{2 \pi}} \int_{0}^{\infty}\left(e^{-i y \theta}+e^{i y \theta}\right) \mathcal{F} f(\eta, y) d y \\
& =\frac{2}{\sqrt{2 \pi}} \int_{0}^{\infty} \cos (y \theta) \mathcal{F} f(\eta, y) d y
\end{aligned}
$$

for $(\eta, \theta) \in \mathbb{H}^{2}$. Now, (B.34), (B.35), and Lemma B.10 imply that

$$
\begin{aligned}
\gamma \mathcal{S}_{\pi, \mu, 1} f & =\frac{1}{\pi} \mathcal{F}^{-1}\left[\int_{\mathbb{R}}\left(\mu^{2}+p(\cdot, \theta)\right)^{-1} \int_{0}^{\infty} \cos (y \theta) \mathcal{F} f(\cdot, y) d y d \theta\right] \\
& =\frac{1}{a_{22}} \mathcal{F}^{-1}\left[\frac{1}{d(\cdot, \mu)} \int_{0}^{\infty} e^{-d(\cdot, \mu) y} \cos (a(\cdot) y) \mathcal{F} f(\cdot, y) d y\right]
\end{aligned}
$$

Consequently, (B.5) yields

$$
\mathcal{T}_{\pi, \mu} \gamma \mathcal{S}_{\pi, \mu, 1} f(x, y)=-\left[\mathcal{F}^{-1} \frac{e^{-\lambda(\cdot, \mu) y}}{d(\cdot, \mu)} k_{f, \mu}\right](x)=-\mathcal{S}_{\pi, \mu, 2} f(x, y)
$$

for all $f \in \mathcal{S}\left(\mathbb{H}^{2}\right)$ and $(x, y) \in \mathbb{H}^{2}$. This completes the proof of Lemma B.8. In order to simplify our notation, let us introduce the following definition:

$$
\mathcal{S}_{\pi, \mu}:=\mathcal{S}_{\pi, \mu, 1}+\mathcal{S}_{\pi, \mu, 2}
$$


Then we prove the following

Corollary B.11. Suppose that $f \in h^{\alpha}\left(\mathbb{H}^{2}\right)$. Then the unique solution of

$$
\left(\mu^{2}+\mathcal{A}_{\pi}\right) u=f \quad \text { in } \quad \mathbb{H}^{2}, \quad u(\cdot, 0)=0 \quad \text { on } \quad \mathbb{R}
$$

in the class $h^{2+\alpha}\left(\mathbb{H}^{2}\right)$ is represented by $u:=\mathcal{S}_{\pi, \mu} f$. Moreover,

$$
\mathcal{S}_{\pi, \mu} \in \mathcal{L}\left(h^{\alpha}\left(\mathbb{H}^{2}\right), h^{2+\alpha}\left(\mathbb{H}^{2}\right)\right)
$$

Proof. Let $f \in h^{\alpha}\left(\mathbb{H}^{2}\right)$ be given and put $u:=\mathcal{S}_{\pi, \mu} f$. It follows from Lemmas B.8 and B.9 that $\mathcal{S}_{\pi, \mu} \in \mathcal{L}\left(h^{\alpha}\left(\mathbb{H}^{2}\right), h^{2+\alpha}\left(\mathbb{H}^{2}\right)\right)$. Hence, $u \in h^{2+\alpha}\left(\mathbb{H}^{2}\right)$. Furthermore, Lemma. B.9 and Lemma B.2 imply that

$$
\mathcal{A}_{\pi, \mu} \mathcal{S}_{\pi, \mu, 1}=\operatorname{id}_{h^{\alpha}\left(\mathbb{H}^{2}\right)} \quad \text { and } \quad \mathcal{A}_{\pi, \mu} \mathcal{T}_{\pi, \mu}=0
$$

respectively. Consequently, by Lemma B.8,

$$
\mathcal{A}_{\pi, \mu} u=\mathcal{A}_{\pi, \mu} \mathcal{S}_{\pi, \mu, 1} f-\mathcal{A}_{\pi, \mu} \mathcal{T}_{\pi, \mu} \gamma \mathcal{S}_{\pi, \mu, 1} f=f
$$

On the boundary we infer from the identity $\gamma \mathcal{T}_{\pi, \mu}=\mathrm{id}_{h^{2+\alpha}}$, see Lemma B.2 and (B.29), that

$$
\gamma u=\gamma \mathcal{S}_{\pi, \mu, 1} f-\gamma \mathcal{T}_{\pi, \mu} \gamma \mathcal{S}_{\pi, \mu, 1} f=0
$$

This shows that $u$ is a solution of (B.36). Again, uniqueness is guaranteed by the maximum principle.

Combining Lemma B.2 and Corollary B.11, we immediately obtain

Corollary B.12. Given $f \in h^{\alpha}\left(\mathbb{H}^{2}\right)$ and $g \in h^{2+\alpha}$, there exists a unique solution of

$$
\left(\mu^{2}+\mathcal{A}_{\pi}\right) u=f \quad \text { in } \quad \mathbb{H}^{2}, \quad u(\cdot, 0)=g \quad \text { on } \quad \mathbb{R} .
$$

It is given by

$$
u=\mathcal{S}_{\pi, \mu} f+\mathcal{T}_{\pi, \mu} g
$$

Remark B.14. It follows from Remark B.5a), (B.28), and the representation (B.29) that there exists a positive constant $C:=C\left(\alpha_{0}, \mu\right)$ such that

$$
\left\|\mathcal{S}_{\pi, \mu}\right\|_{\mathcal{L}\left(h^{\alpha}\left(\mathbb{H}^{2}\right), h^{2+\alpha}\left(\mathbb{H}^{2}\right)\right)}+\left\|\mathcal{T}_{\pi, \mu}\right\|_{\mathcal{L}\left(h^{2+\alpha}, h^{2+\alpha}\left(\mathbb{H}^{2}\right)\right)} \leq C .
$$

\section{Proof of Theorem 3.5}

Throughout this section we assume that $K$ is a compact subset of $h_{\mathfrak{a}}^{2+\alpha}$. Also assume that $\lambda_{0}, \mu_{0}, \mu_{1} \in(0, \infty)$ with $\mu_{0}<\mu_{1}$ and pick $\lambda \in\left[0, \lambda_{0}\right], \mu \in\left[\mu_{0}, \mu_{1}\right]$. 
a) Let us start with the classical estimates of Agmon-Douglis-Nirenberg for elliptic equations. It follows from [1, Theorem 7.3] and Lemma 2.2 that there exists a positive constant $C:=C\left(K, \lambda_{0}, \mu_{0}, \mu_{1}, \alpha, c\right)$ such that

$$
\begin{gathered}
\|u\|_{2+\alpha, \Omega} \leq C\left(\|(\lambda+\mathcal{A}(g)) u\|_{\alpha, \Omega}+\left\|\gamma_{0} u\right\|_{2+\alpha}\right. \\
\left.+\left\|(c+g) \mathcal{B}_{1}(g) u\right\|_{1+\alpha}+\|u\|_{0, \Omega}\right) \\
\|u\|_{2+\alpha, \Omega} \leq C\left(\|(\lambda+\mathcal{A}(g)) u\|_{\alpha, \Omega}+\left\|\left(\mu \gamma_{0}+\mathcal{B}_{0}(g)\right) u\right\|_{1+\alpha}\right. \\
\left.+\left\|\mathcal{B}_{1}(g) u\right\|_{1+\alpha}+\|u\|_{0, \Omega}\right)
\end{gathered}
$$

for all $u \in h^{2+\alpha}(\Omega), g \in K, \lambda \in\left[0, \lambda_{0}\right]$, and $\mu \in\left[\mu_{0}, \mu_{1}\right]$.

b) In a next step, we improve the above results by estimating $\|u\|_{0, \Omega}$ in terms of $\|(\lambda+\mathcal{A}(g)) u\|_{\alpha, \Omega}, \quad\left\|\gamma_{0} u\right\|_{2+\alpha},\left\|\left(\mu \gamma_{0}+\mathcal{B}_{0}(g) u\right)\right\|_{1+\alpha}$, and $\left\|\mathcal{B}_{1}(g) u\right\|_{1+\alpha}$. More precisely, we assert that there exists a positive constant $C:=C\left(K, \lambda_{0}, \mu_{0}, \mu_{1}, \alpha, c\right)$ such that

$$
\begin{aligned}
& \|u\|_{2+\alpha, \Omega} \leq C\left(\|(\lambda+\mathcal{A}(g)) u\|_{\alpha, \Omega}+\left\|\gamma_{0} u\right\|_{2+\alpha}+\left\|(c+g) \mathcal{B}_{1}(g) u\right\|_{1+\alpha}\right) \\
& \|u\|_{2+\alpha, \Omega} \leq C\left(\|(\lambda+\mathcal{A}(g)) u\|_{\alpha, \Omega}+\left\|\left(\mu \gamma_{0}+\mathcal{B}_{0}(g)\right) u\right\|_{1+\alpha}+\left\|\mathcal{B}_{1}(g) u\right\|_{1+\alpha}\right)
\end{aligned}
$$

for all $u \in h^{2+\alpha}(\Omega), g \in K, \lambda \in\left[0, \lambda_{0}\right]$, and $\mu \in\left[\mu_{0}, \mu_{1}\right]$.

In order to further economize our notation we suppress in the following the $g$ dependence of the differential operators. Also let $\mathcal{A}_{\lambda}:=\lambda+\mathcal{A}$ and $\mathcal{B}_{0, \mu}:=\mu+\mathcal{B}_{0}$. Furthermore, we only present an explicit proof of the second estimate. The first one can be verified analogously.

Suppose that $M, \alpha$, and $\beta$ are positive constants and set $w(z):=M-\beta h(z)$, where $h(z):=e^{-\alpha y}$ for $z=(x, y) \in \bar{\Omega}$. An elementary calculation shows

$$
\left(\mathcal{A}_{\lambda}, \mathcal{B}_{0, \mu}, \mathcal{B}_{1}\right) w=\left(\lambda w+\beta\left[\alpha^{2} a_{22}+\alpha a_{2}\right] h, \mu w+\alpha \beta b_{2,0} \gamma_{0} h, \alpha \beta b_{2,1} \gamma_{1} h\right)
$$

Let

$$
\underline{\alpha}:=\inf _{g \in K} \frac{1}{1+\left(c+\|g\|_{0}\right)^{2}+\left\|g_{x}\right\|_{0}^{2}} .
$$

Then $\underline{\alpha}$ is positive, due to the compactness of $K$. Moreover, it follows from Lemma 2.2 that

$$
a_{22}(z) \geq \underline{\alpha} \text { and } b_{2,1}(x) \geq \underline{\alpha}
$$

for all $z \in \bar{\Omega}, x \in \mathbb{R}$. Hence, (C.3) implies that the constants $M, \alpha$, and $\beta$ can be chosen in such a way that we find positive constants $\bar{w}, \underline{w}, \underline{c}, \kappa_{0}$, and $\kappa_{1}$ with

$$
\begin{aligned}
& \bar{w} \geq w \geq \underline{w}>0 \text { in } \bar{\Omega}, \quad \mathcal{A}_{\lambda} w \geq \underline{c}>0 \text { in } \bar{\Omega}, \\
& \mathcal{B}_{0, \mu} w \geq \kappa_{0}>0 \text { on } \Gamma_{0}, \quad \mathcal{B}_{1} w \geq \kappa_{1}>0 \text { on } \Gamma_{1} .
\end{aligned}
$$

Now let $u \in h^{2+\alpha}(\Omega)$ be given and set $f:=\mathcal{A}_{\lambda} u, h_{0}:=\mathcal{B}_{0, \mu} u, h_{1}:=\mathcal{B}_{1} u$, and $v:=\frac{u}{w}$. Since $|v(z)| \rightarrow 0$ as $|z| \rightarrow \infty$, it follows that $|v|$ attains its maximum in $\bar{\Omega}$, say in $z_{0}$. 
ii) Suppose that $z_{0} \in \Omega$ and that $v\left(z_{0}\right)$ is the nonnegative maximum. Then

$$
\nabla v\left(z_{0}\right)=0 \quad \text { and } \quad-\mathcal{A} v\left(z_{0}\right)=\sum_{j, k=1}^{2} a_{j k}\left(z_{0}\right) \partial_{j} \partial_{k} v\left(z_{0}\right) \leq 0
$$

Also observe that

$$
\frac{f}{w}=\frac{\mathcal{A}_{\lambda} u}{w}=\mathcal{A} v-\frac{2}{w} \sum_{j, k=1}^{2} a_{j k} \partial_{j} w \partial_{k} v+\frac{v}{w} \mathcal{A}_{\lambda} w
$$

Hence $(C .6)$ yields

$$
\frac{f\left(z_{0}\right)}{w\left(z_{0}\right)} \geq \frac{v\left(z_{0}\right)}{w\left(z_{0}\right)} \mathcal{A}_{\lambda} w\left(z_{0}\right) \geq \underline{c} \frac{v\left(z_{0}\right)}{w\left(z_{0}\right)}
$$

which shows that $v\left(z_{0}\right) \leq c^{-1} f\left(z_{0}\right)$. If $v\left(z_{0}\right)$ is the negative minimum an analogous argument implies that $\left|v\left(z_{0}\right)\right| \leq \underline{c}^{-1}\left|f\left(z_{0}\right)\right|$. Summarizing, we find that

$$
\|u\|_{0, \Omega} \leq\|v\|_{0, \Omega}\|w\|_{0, \Omega} \leq \frac{\bar{w}}{\underline{c}}\|f\|_{0, \Omega}=\frac{\bar{w}}{\underline{c}}\left\|\mathcal{A}_{\lambda} u\right\|_{\emptyset, \Omega} \leq \frac{\bar{w}}{\underline{c}}\left\|\mathcal{A}_{\lambda} u\right\|_{\alpha, \Omega}
$$

if $z_{0}$ lies in $\Omega$.

iii) Now suppose that $z_{0}=\left(x_{0}, 0\right)$ lies on $\Gamma_{0}$ and that $v\left(z_{0}\right)$ is the nonnegative maximum. Then, necessarily it holds that

$$
\mathcal{B}_{0} v\left(x_{0}\right) \geq 0
$$

Since we also have

$$
\frac{h_{0}}{w}=\frac{\mathcal{B}_{0, \mu} u}{w}=\mathcal{B}_{0} v+\frac{v}{w} \mathcal{B}_{0, \mu} w
$$

(C.8) and (C.5) imply that

$$
\|v\|_{0}=v\left(z_{0}\right)=\frac{w\left(z_{0}\right)}{\mathcal{B}_{0, \mu} w\left(z_{0}\right)}\left[\frac{h_{0}\left(x_{0}\right)}{w\left(z_{0}\right)}-\mathcal{B}_{0} v\left(x_{0}\right)\right] \leq \frac{h_{0}\left(x_{0}\right)}{\kappa_{0}} \leq \frac{\left\|h_{0}\right\|_{0}}{\kappa_{0}} .
$$

A similar argument shows that $(C .9)$ remains true if $v\left(z_{0}\right)$ is the negative minimum. From (C.9) we now infer that

$$
\|u\|_{0, \Omega} \leq\|v\|_{0, \Omega}\|w\|_{0, \Omega} \leq \frac{\bar{w}}{\kappa_{0}}\left\|h_{0}\right\|_{0} \leq \frac{\bar{w}}{\kappa_{0}}\left\|\mathcal{B}_{0, \beta} u\right\|_{1+\alpha}
$$

iv) If $z_{0}$ lies on $\Gamma_{1}$ an analogous consideration as in ii) shows that

$$
\|u\|_{0, \Omega} \leq \frac{\bar{w}}{\kappa_{1}}\left\|h_{1}\right\|_{0} \leq \frac{\bar{w}}{\kappa_{1}}\left\|\mathcal{B}_{1} u\right\|_{1+\alpha}
$$

It remains to combine $(C .7),(C .8)$, and (C.11) to complete the proof of b). 
c) Recall that $0 \in h_{\mathfrak{X}}^{2+\alpha}$ and observe $\mathcal{A}(0)=-\partial_{1}^{2}-\frac{1}{c^{2}} \partial_{2}^{2}, \mathcal{B}_{0}(0)=-\frac{1}{c} \partial_{2}$, and $\mathcal{B}_{1}(0)=\frac{1}{c} \partial_{2}$. Since the coefficients of these operators are constant it is well-known that the corresponding boundary value problem on the strip $\Omega=\mathbb{R} \times(0,1)$ is wellposed in $W_{2}^{2}(\Omega)$. Indeed, the existence of a unique weak solution, i.e., a solution in $W_{2}^{1}(\Omega)$, follows from a standard argument based on the Lax-Milgram Lemma. Well-known regularity results for elliptic equations, e.g. Theorem 8.12, p. 176 in [14] guarantee now that the solution belongs to $W_{2}^{2}(\Omega)$. Hence, using Theorem 13.1 in [2], we find that

$$
\begin{aligned}
& \left(\mathcal{A}(0), \gamma_{0}, c \mathcal{B}_{1}(0)\right) \in \operatorname{Isom}\left(W_{2}^{4}(\Omega), W_{2}^{2}(\Omega) \times W_{2}^{3+\frac{1}{2}} \times W_{2}^{2+\frac{1}{2}}\right) \\
& \left(\mathcal{A}(0), \mathcal{B}_{0, \mu_{0}}(0), \mathcal{B}_{1}(0)\right) \in \operatorname{Isom}\left(W_{2}^{4}(\Omega), W_{2}^{2}(\Omega) \times W_{2}^{2+\frac{1}{2}} \times W_{2}^{2+\frac{1}{2}}\right) .
\end{aligned}
$$

Our goal is to prove that these boundary value problems are well-posed in little Hölder spaces too. Thus, let $\left(f, h_{0}, h_{1}\right) \in h^{\alpha}(\Omega) \times h^{2+\alpha} \times h^{1+\alpha}$ be given and observe that

$$
W_{2}^{2}(\Omega) \times W_{2}^{3+\frac{1}{2}} \times W_{2}^{2+\frac{1}{2}} \stackrel{d}{\hookrightarrow} h^{\alpha}(\Omega) \times h^{2+\alpha} \times h^{1+\alpha},
$$

due to Sobolev's embedding theorem, cf. Theorem 11.5 in [2]. Hence there is a sequence $\left(f_{n}, h_{0, n}, h_{1, n}\right) \in W_{2}^{2}(\Omega) \times W_{2}^{3+\frac{1}{2}} \times W_{2}^{2+\frac{1}{2}}$ such that

$$
\left(f_{n}, h_{0, n}, h_{1, n}\right) \rightarrow\left(f, h_{0}, h_{1}\right) \text { in } h^{\alpha}(\Omega) \times h^{2+\alpha} \times h^{1+\alpha}
$$

as $n \rightarrow \infty$. Define $u_{n}:=\left(\mathcal{A}(0), \gamma_{0}, c \mathcal{B}_{1}(0)\right)^{-1}\left(f_{n}, h_{0, n}, h_{1, n}\right)$ and observe that $u_{n} \in$ $W_{2}^{4}(\Omega)$. Since also $W_{2}^{4}(\Omega) \hookrightarrow h^{2+\alpha}(\Omega)$ it follows from the a priori estimates $(C, 2)$ and from $(C .14)$ that $u_{n}$ converges towards a solution $u$ of

$$
\mathcal{A}(0) u=f \text { in } \Omega, \quad \gamma_{0} u=h_{0} \text { on } \Gamma_{0}, \quad c \mathcal{B}_{1}(0) u=h_{1} \text { on } \Gamma_{1}
$$

in $h^{2+\alpha}$ as $n \rightarrow \infty$. This shows that

$$
\left(\mathcal{A}(0), \gamma_{0}, c \mathcal{B}_{1}(0)\right) \in \mathcal{L}\left(h^{2+\alpha}(\Omega), h^{\alpha}(\Omega) \times h^{2+\alpha} \times h^{1+\alpha}\right)
$$

is surjective. But we already know from (C.2) that this operator is injective. Hence

$$
\begin{aligned}
& \left(\mathcal{A}(0), \gamma_{0}, c \mathcal{B}_{1}(0)\right) \in \operatorname{Isom}\left(h^{2+\alpha}(\Omega), h^{\alpha}(\Omega) \times h^{2+\alpha} \times h^{1+\alpha}\right), \\
& \left(\mathcal{A}(0), \mathcal{B}_{0, \mu_{0}}(0), \mathcal{B}_{1}(0)\right) \in \operatorname{Isom}\left(h^{2+\alpha}(\Omega), h^{\alpha}(\Omega) \times h^{1+\alpha} \times h^{1+\alpha}\right),
\end{aligned}
$$

with obvious modifications for the second assertion.

d) Given $g \in h_{\mathfrak{a}}^{2+\alpha}$ and $t \in[0,1]$, define

$$
\begin{aligned}
& H_{0}(g, t):=\left(t \lambda+\mathcal{A}(t g), \gamma_{0},(c+t g) \mathcal{B}_{1}(t g)\right) \\
& H_{1}(g, t):=\left(t \lambda+\mathcal{A}(t g),(1-t) \mu_{0}+t \mu+\mathcal{B}_{0}(t g), \mathcal{B}_{1}(t g)\right)
\end{aligned}
$$


Observe that

$$
\begin{aligned}
& H_{0}(g, 0)=\left(\mathcal{A}(0), \gamma_{0}, c \mathcal{B}_{1}(0)\right), \quad H_{0}(g, 1)=\left(\mathcal{A}_{\lambda}(g), \gamma_{0},(c+g) \mathcal{B}_{1}(g)\right) \\
& H_{1}(g, 0)=\left(\mathcal{A}(0), \mathcal{B}_{0, \mu_{0}}(0), \mathcal{B}_{1}(0)\right), H_{1}(g, 1)=\left(\mathcal{A}_{\lambda}(g), \mathcal{B}_{0, \mu}(g), \mathcal{B}_{1}(g)\right)
\end{aligned}
$$

Moreover, it follows from Lemma 3.4 that

$$
H_{i}(g, \cdot) \in C\left([0,1], \mathcal{L}\left(h^{2+\alpha}(\Omega), h^{\alpha}(\Omega) \times h^{2-i+\alpha} \times h^{1+\alpha}\right), i=0,1 .\right.
$$

Now let $g \in h_{\mathfrak{A}}^{2+\alpha}$ be given and set $K_{0}:=\{t g ; t \in[0,1]\}$. Observe that $K_{0} \subset h_{\mathfrak{a}}^{2+\alpha}$ and that $K_{0}$ is compact. Hence, we infer from b) that there is a positive constant $C$ such that

$$
\|u\|_{2+\alpha, \Omega} \leq C\left\|H_{i}(g, t) u\right\|_{h^{\alpha}(\Omega) \times h^{2-i+\alpha} \times h^{1+\alpha}}
$$

for all $u \in h^{2+\alpha}(\Omega), t \in[0,1]$, and $i=0,1$. Now an obvious modification of Theorem 5.2 in [14] applied to (C.15)-(C.17) yields that

$$
\begin{aligned}
& \left(\lambda+\mathcal{A}(g), \gamma_{0},(c+g) \mathcal{B}_{1}(g)\right) \in \operatorname{Isom}\left(h^{2+\alpha}(\Omega), h^{\alpha}(\Omega) \times h^{2+\alpha} \times h^{1+\alpha}\right) \\
& \left(\lambda+\mathcal{A}(g), \mu \gamma_{0}+\mathcal{B}_{0}(g), \mathcal{B}_{1}(g)\right) \in \operatorname{Isom}\left(h^{2+\alpha}(\Omega), h^{\alpha}(\Omega) \times h^{1+\alpha} \times h^{1+\alpha}\right) .
\end{aligned}
$$

This completes the prove of Theorem 3.5.

\section{References}

[1] S. AGMON, A. DOUGLIS, L. NIRENBERG, Estimates near the boundary for solutions of elliptic partial differential equations satisfying general boundary conditions. I, Comm. Pure Appl. Math. 12, 623-727 (1959)

[2] H. AMANN, Existence and regularity for semilinear parabolic evolution equations, Ann. Scuola Norm. Pisa, (4) XI, 593-676 (1984).

[3] H. AMANN, Dynamic theory of quasilinear parabolic equations. I, Nonlinear Anal., TMA 12, 895-919 (1988)

[4] H. AMANN Linear and Quasilinear Parabolic Problems, Book in preparation, 1994

[5] H. AMANN, J. ESCHER, Strongly continuous dual semigroups, to appear in Ann. Mat. Pura Appl.

[6] S. B. ANGENENT, Nonlinear analytic semiflows, Proc. Roy. Soc. Edinburgh 115 A, 91-107 (1990)

[7] J. BEAR, Y. BACHMAT, Introduction to Modeling of Transport Phenomena in Porous Media, Kluwer Academic Publisher, Boston, 1990 
[8] F. E. BROWDER, On the spectral theory of elliptic operators I, Math. Ann. 142, 22-130 (1961)

[9] G. DA PRATO, P. GRISVARD, Equations d'évolution abstraites nonlinéaires de type parabolique, Ann. Mat. Pura Appl., (4) 120, 329-396 (1979)

[10] C. M. ELLIOTT, J. R. OCKENDON, Weak and Variational Methods for Moving Boundary Problems, Pitman, Boston, 1982

[11] J. ESCHER, Nonlinear elliptic systems with dynamic boundary conditions, Math. Z. 210, 413-439 (1992)

[12] J. ESCHER, The Dirichlet-Neumann operator on continuous functions, Ann. Scuola Norm. Pisa, (4) XXI, 235-266 (1994)

[13] A. FRIEDMAN, B. HU, The Stefan problem with kinetic condition at the free boundary, Ann. Scuola Norm. Pisa, (4) XLIV, 87-111 (1992)

[14] D. GILBARG, N.S. TRUDINGER, Elliptic Partial Differential Equations of Second Order, Springer-Verlag, Berlin, 1977

[15] G. GRUBB, Functional Calculus of Pseudo-Differential Boundary Problems, Birkhäuser-Verlag, Basel, 1986

[16] T. HINTERMANN, Evolution equations with dynamic boundary conditions, Proc. Roy. Soc. Edinburgh 113 A, 43-60 (1989)

[17] B. HUNT, Vertical recharge of unconfined aquifer. J. Hydr. Divn. 97, 10171030 (1971)

[18] A. LUNARDI. On the local dynamical system associated to a fully nonlinear abstract parabolic equation. In Nonl. Anal. and Appl., ed. Lakshmikantham, M. Dekker, New York, 319-326 (1987)

[19] A. LUNARDI, Analyticity of the maximal solution of an abstract nonlinear parabolic equation, Nonlinear Anal. TMA, 6, 503-521 (1982)

[20] A. LUNARDI, Analytic Semigroups and Optimal Regularity in Parabolic Equations, Birkhäuser-Verlag, Basel, 1994

[21] H. KAWARADA, H. KOSHIGOE, Unsteady flow in porous media with a free surface, Japan J. Indust. Appl. Math., 8, 41-82 (1991)

[22] B. E. PETERSON, Introduction to Fourier Transform and Pseudo-Differential Operators, Pitman, Boston, London, 1983

[23] M. H. PROTTER, H. F. WEINBERGER, Maximum Principles in Differential Equations, Springer-Verlag, Berlin, 1984 
[24] G. SIMONETT, Zentrumsmannigfaltigkeiten für quasilineare parabolische Gleichungen. Institut für angewandte Analysis und Stochastik, Report Nr. 2, Berlin, 1992

[25] G. SIMONETT, Quasilinear parabolic equations and semiflows. In Evolution Equations, Control Theory, and Biomathematics, Lecture Notes in Pure and Appl. Math. M. Dekker, New York, 523-536 (1994)

[26] E. SINESTRARI, Continuous interpolation spaces and spatial regularity in nonlinear Volterra integrodifferential equations, J. Integral Equs. 5, 287-308 (1983).

[27] E. M. STEIN, Singular Integrals and Differentiability Properties of Functions, University Press, Princeton, 1970

[28] H. TRIEBEL, Theory of Function Spaces, Birkhäuser-Verlag, Basel, 1983

[29] H. TRIEBEL, Theory of Function Spaces II, Birkhäuser-Verlag, Basel, 1992

Received May 16, 1994 